\title{
Métodos de Diagnóstico em Modelos Logísticos Trinomiais
}

\author{
José Alberto P. Silva \\ Dissertação apresentada \\ $\mathrm{aO}$ \\ Instituto de Matemática e Estatística \\ da \\ Universidade de São Paulo \\ para \\ obtenção do grau \\ de \\ Mestre em Estatística \\ Área de concentração: Estatística \\ Orientador: Gilberto A. Paula
}

São Paulo - junho de 2003 


\section{Resumo}

Os modelos logísticos trinomiais podem ser interpretados como uma extensão natural do modelo logístico binomial para situações em que a resposta admite apenas três resultados possíveis. Introduzimos inicialmente os modelos logísticos trinomiais e discutimos em seguida alguns aspectos inferenciais, tais como estimação e testes. Medidas de qualidade do ajuste são também apresentadas. Contudo, o principal foco deste trabalho é a apresentação de métodos de diagnóstico. Mostramos que as técnicas usuais de diagnóstico desenvolvidas para o modelo logístico binomial podem ser adaptadas para o caso trinomial. O desenvolvimento de métodos diretos para o modelo logístico trinomial é mais complexo do ponto de vista computacional, embora seja sempre possível. Discutimos alguns desses métodos, dentre os quais, o desenvolvimento de resíduos, de métodos para detectar pontos de alavanca, métodos de deleção de pontos e influência local. Comparamos os métodos adaptados com alguns métodos diretos através de exemplos. 


\section{Abstract}

The trinomial logistic models can be interpreted as a natural extension of the traditional binomial logistic model to situations in which the response allows only three possible results. We first introduce the trinomial logistic models and then some inferential aspects, such as estimation and hypothesis testing are discussed. Goodness-of-fit measures are also given. However, the aim of this work is the presentation of some diagnostic procedures for trinomial logistic models. We show that methods developed for binomial logistic models can adapted straightforward for trinomial models. The development of direct methods, even though possible, in general requires more complex calculation. Some of these direct methods, such as evaluation of residuals, measures of high leverage points, deletion methods and local influence are presented. Comparisons between adapted and direct methods are made via examples with real data. 


\section{Sumário}

$\begin{array}{ll}\text { Resumo } & 3\end{array}$

$\begin{array}{ll}\text { Abstract } & 5\end{array}$

1 Modelos Logísticos Trinomiais $\quad 9$

1.1 Introdução . . . . . . . . . . . . . . . . . . . . . . . . . . . 9

1.2 Modelo Logístico Trinomial . . . . . . . . . . . . . . . . . . 10

1.3 Função Escore . . . . . . . . . . . . . . . . . . . . . . . . . 13

1.4 Matriz de Informação . . . . . . . . . . . . . . . . . . . . . . . . . . . . . . 14

1.5 Processo Iterativo . . . . . . . . . . . . . . . . . . 15

1.6 Função Desvio . . . . . . . . . . . . . . . . . . . . . . . 16

1.7 Estatística de Hosmer e Lemeshow . . . . . . . . . . . . . . . . . . . . . 18

1.8 Resultados Inferenciais . . . . . . . . . . . . . . . . . . . . . . . . . 19

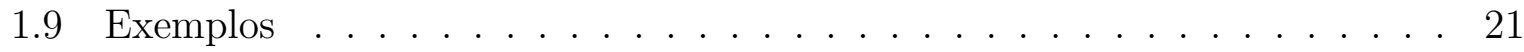

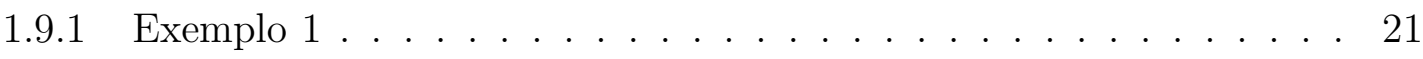

1.9.2 Exemplo 2 . . . . . . . . . . . . . . . . . . . . 31

2 Métodos de Diagnóstico 37

2.1 Introdução . . . . . . . . . . . . . . . . . . . . . . . . . 37

2.2 Pontos de Alavanca . . . . . . . . . . . . . . . . . . . . . . 38

2.3 Deleção de Pontos . . . . . . . . . . . . . . . . . . . . . . . . . 42 
2.4 Influência Local . . . . . . . . . . . . . . . . . . . . . . . . . . . . . 45

2.5 Influência Local Total . . . . . . . . . . . . . . . . . . . . . . . . . . 49

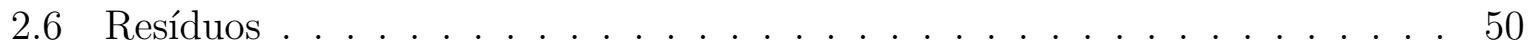

3 Aplicações $\quad 53$

3.1 Introdução . . . . . . . . . . . . . . . . . . . . . . 53

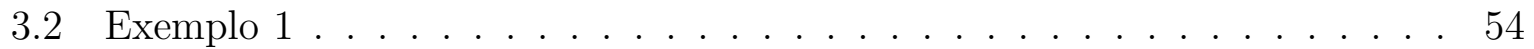

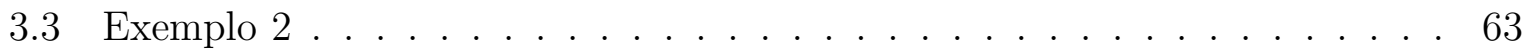

4 Considerações Finais $\quad 75$

$\begin{array}{ll}\text { Apêndice } & 77\end{array}$

$\begin{array}{ll}\text { Bibliografia } & 95\end{array}$ 


\section{Capítulo 1}

\section{Modelos Logísticos Trinomiais}

\subsection{Introdução}

Regressão logística é mais frequentemente empregada para modelar o relacionamento entre uma variável com resposta dicotômica (binomial) e um conjunto de variáveis explicativas, porém com algumas modificações ela pode também ser utilizada quando a variável resposta é policotômica (multinomial). A extensão do modelo e métodos binomial para multinomial é facilmente ilustrado quando a variável resposta tem três categorias (trinomial). Generalizações para respostas com mais de três categorias é um problema mais notacional que conceitual. Em nosso trabalho consideramos a situação em que a variável resposta assume três categorias (trinomial).

Neste capítulo apresentaremos inicialmente alguns aspectos inferenciais do modelo logístico trinomial e em seguida como ilustração dois exemplos com dados reais serão apresentados. No capítulo 2 discutiremos algumas técnicas de diagnóstico que podem ser aplicadas no caso trinomial. Parte dessas técnicas são adaptações diretas das técnicas usuais da regressão logística binomial. As demais técnicas são desenvolvidas diretamente para o modelo logístico trinomial. No capítulo 3 aplicações e comparações dessas técnicas são feitas nos dois exemplos ilustrativos apresentados no capítulo 1. Conclusões e sugestões são apresntadas no último capítulo. 


\subsection{Modelo Logístico Trinomial}

No desenvolvimento de modelos para respostas policotômicas precisamos ter conhecimento de sua escala de medida. A maioria das aplicações, inclusive o nosso foco, envolve uma variável resposta com escala nominal. Quando trabalhamos com o modelo logístico binomial a variável resposta assume os valores 0 e 1 . Sem perda de generalidades vamos supor o modelo logístico trinomial. Seja Y uma variável aleatória categórica que assume os valores $\mathrm{Y}=0,1,2$ tais que $\pi_{0}=P(Y=0), \pi_{1}=P(Y=1)$, e $\pi_{2}=P(Y=2)$ sujeito à restrição $\pi_{0}+\pi_{1}+\pi_{2}=1$.

Para construir a função de probabilidade, é conveniente utilizar três variáveis binárias codificadas como 0 ou 1 , tais que

$$
\begin{aligned}
& Z_{0}=1, \quad Z_{1}=0, \quad Z_{2}=0 \quad \text { se } \quad \mathrm{Y}=0, \\
& Z_{0}=0, \quad Z_{1}=1, \quad Z_{2}=0 \quad \text { se } \quad \mathrm{Y}=1 \quad \text { e } \\
& Z_{0}=0, \quad Z_{1}=0, \quad Z_{2}=1 \quad \text { se } \quad Y=2 \text {. }
\end{aligned}
$$

Note que $Z_{0}+Z_{1}+Z_{2}=1$. Portanto, a função de probabilidade de $\mathbf{Z}=\left(Z_{0}, Z_{1}, Z_{2}\right)^{T}$ fica dada por

$$
g\left(z ; \pi_{0}, \pi_{1}, \pi_{2}\right)=\pi_{0}^{z_{0}} \pi_{1}^{z_{1}} \pi_{0}^{z_{2}}
$$

Vamos supor agora que a variável aleatória Y é observada independentemente em n unidades experimentais, sendo que para a i-ésima unidade tem-se $P\left(Y_{i}=0\right)=\pi_{0 i}, P\left(Y_{i}=\right.$ $1)=\pi_{1 i}$ e $P\left(Y_{i}=2\right)=\pi_{2 i}$ e a variável aleatória $\mathbf{Z}_{i}=\left(Z_{0 i}, Z_{1 i}, Z_{2 i}\right)^{T}, i=1,2, \ldots, n$, com as mesmas atribuições de $\mathbf{Z}$.

A função de probabilidade conjunta de $\left(\mathbf{Z}_{1}, \mathbf{Z}_{2}, \ldots, \mathbf{Z}_{n}\right)$ pode ser expressa na forma

$$
g\left(\mathbf{z}_{1}, \ldots, \mathbf{z}_{n} ; \boldsymbol{\pi}_{1}, \ldots, \boldsymbol{\pi}_{n}\right)=\prod_{i=1}^{n} \pi_{0 i}^{z_{0 i}} \pi_{1 i}^{z_{1 i}} \pi_{2 i}^{z_{2 i}},
$$

em que

$$
\mathbf{z}_{i}=\left(z_{0 i}, z_{1 i}, z_{2 i}\right)^{T} \text { e } \boldsymbol{\pi}_{i}=\left(\pi_{o i}, \pi_{1 i}, \pi_{2 i}\right)^{T}
$$


sujeito às restrições

$$
\pi_{0 i}+\pi_{1 i}+\pi_{2 i}=1 \text { e } \sum_{i=1}^{n}\left(z_{0 i}+z_{1 i}+z_{2 i}\right)=n .
$$

Quando trabalhamos com o modelo logístico binomial a resposta é parametrizada em termos do logito de $Y=1$ versus $Y=0$. No caso do modelo trinomial teremos dois logitos: um para $\mathrm{Y}=1$ versus $\mathrm{Y}=0$ e outro para $\mathrm{Y}=2$ versus $\mathrm{Y}=0$. Teoricamente podemos comparar quaisquer pares de logitos; porém, a extensão óbvia do caso binomial é utilizar o logito $Y=2$ versus $Y=0$ para a segunda função. Assim, o grupo codificado como $Y=0$ servirá como um valor de referência para a resposta. O logito para comparar $\mathrm{Y}=2$ versus $Y=1$ pode ser obtido como a diferença entre os logitos $Y=2$ versus $Y=0$ e $Y=1$ versus $Y=0$.

É usual (vide, por exemplo, Hosmer e Lemeshow, 1989, Cap. 8) que a parte sistemática do modelo assuma a forma

$$
\begin{aligned}
& \log \left\{\frac{\pi_{1 i}}{\pi_{0 i}}\right\}=\eta_{1 i}=\mathbf{x}_{i}^{T} \boldsymbol{\beta}_{1} \\
& \log \left\{\frac{\pi_{2 i}}{\pi_{0 i}}\right\}=\eta_{2 i}=\mathbf{x}_{i}^{T} \boldsymbol{\beta}_{2},
\end{aligned}
$$

em que $\mathbf{x}_{i}=\left(x_{i 1}, x_{i 2}, \ldots, x_{i p}\right)^{T}$ denota os valores de $p$ variáveis explicativas observadas em cada unidade experimental, $\boldsymbol{\beta}_{1}=\left(\beta_{11}, \beta_{12}, \ldots, \beta_{1 p}\right)^{T}$ e $\boldsymbol{\beta}_{2}=\left(\beta_{21}, \beta_{22}, \ldots, \beta_{2 p}\right)^{T}$ são parâmetros desconhecidos a serem estimados. Note que temos dois sub-modelos logísticos neste caso com a mesma relação funcional na parte sistemática, porém com parâmetros diferentes.

Das equações (1.1) e (1.2) mostra-se facilmente que

$$
\begin{aligned}
\pi_{0 i} & =\frac{1}{1+e^{\eta_{1 i}}+e^{\eta_{2 i}}}, \\
\pi_{1 i} & =\frac{e^{\eta_{1 i}}}{1+e^{\eta_{1 i}}+e^{\eta_{2 i}}} \quad \mathrm{e} \\
\pi_{2 i} & =\frac{e^{\eta_{2 i}}}{1+e^{\eta_{1 i}}+e^{\eta_{2 i}}} .
\end{aligned}
$$


Para ilustar, considere o exemplo em que Y representa o estado nutricional de um recémnascido e as respostas são codificadas da seguinte forma:

$$
\begin{array}{ll}
Y=0 & \text { se o estado nutricional está abaixo do esperado, } \\
Y=1 & \text { se o estado nutricional está na média esperada e } \\
Y=2 & \text { se o estado nutricional está acima do esperado. }
\end{array}
$$

Vamos supor ainda que sejam observadas as variáveis explicativas $X_{1}$ e $X_{2}$ tais que $X_{1}=1$ se a criança é pré-matura e $X_{1}=0$ caso contrário, enquanto $X_{2}$ representa o peso ao nascer. Pode-se propor, por exemplo, o seguinte modelo logístico:

$$
\begin{gathered}
\log \left\{\frac{\pi_{1 i}}{\pi_{0 i}}\right\}=\beta_{10}+\beta_{11} x_{1 i}+\beta_{12} x_{2 i} \\
\mathrm{e} \\
\log \left\{\frac{\pi_{2 i}}{\pi_{0 i}}\right\}=\beta_{20}+\beta_{21} x_{1 i}+\beta_{22} x_{2 i},
\end{gathered}
$$

em que $\pi_{0 i}, \pi_{1 i}$ e $\pi_{2 i}$ representam, respectivamente, as probabilidades

$$
P\left(Y_{i}=0\right), \quad P\left(Y_{i}=1\right) \text { e } P\left(Y_{i}=2\right), \quad i=1, \ldots, n
$$

Pode-se definir algumas razões de chances. Por exemplo, a razão de chances entre prematuro e não-prematuro ter nível nutricional médio supondo o mesmo peso para as crianças $\left(X_{2}=a\right)$ fica dada por

$$
\psi_{1}=\left\{\frac{P\left(Y=1 \mid X_{1}=1, X_{2}=a\right)}{P\left(Y=1 \mid X_{1}=0, X_{2}=a\right)}\right\}=\frac{e^{\beta_{10}+\beta_{11}+\beta_{12} a}}{e^{\beta_{10}+\beta_{12} a}}=e^{\beta_{11}} .
$$

Similamente, a razão de chances entre prematuro e não-prematuro ter nível alto supondo o mesmo peso para os recém-nascidos fica dada por

$$
\psi_{2}=\left\{\frac{P\left(Y=2 \mid X_{1}=1, X_{2}=a\right)}{P\left(Y=2 \mid X_{1}=0, X_{2}=a\right)}\right\}=e^{\beta_{21}} .
$$


Por outro lado, se os pesos dos recém-nascidos forem diferentes $\left(X_{2}=b\right.$ para pré-maturo e $X_{2}=a$ para não pré-maturo) as razões de chances ficam, respectivamente, dadas por

$$
\psi_{1}=e^{\beta_{11}+\beta_{12}(b-a)} \text { e } \psi_{2}=e^{\beta_{21}+\beta_{22}(b-a)},
$$

dependendo, nesse caso, da diferença de pesos $(b-a)$. Assim, uma vantagem da utilização do modelo logístico é a facilidade de interpretação das razões de chances, as quais ficam expressas em função de coeficientes da parte sistemática do modelo.

\subsection{Função Escore}

Vamos obter a função escore para os parâmetros $\boldsymbol{\beta}_{1}$ e $\boldsymbol{\beta}_{2}$ da parte sistemática do modelo logístico trinomial.

O logaritmo da função de verossilhança de $\boldsymbol{\beta}=\left(\boldsymbol{\beta}_{1}^{T}, \boldsymbol{\beta}_{2}^{T}\right)^{T}$ fica dado por

$$
\begin{aligned}
\mathrm{L}(\boldsymbol{\beta}) & =\log \left\{g\left(\mathbf{z}_{1}, \ldots, \mathbf{z}_{n} ; \boldsymbol{\pi}_{1}, \ldots, \boldsymbol{\pi}_{n}\right)\right\}=\sum_{i=1}^{n}\left\{z_{0 i} \log \pi_{0 i}+z_{1 i} \log \pi_{1 i}+z_{2 i} \log \pi_{2 i}\right\} \\
& =\sum_{i=1}^{n}\left\{z_{1 i} \eta_{1 i}+z_{2 i} \eta_{2 i}-\left(z_{0 i}+z_{1 i}+z_{2 i}\right) \times \log \left(1+e^{\eta_{1 i}}+e^{\eta_{2 i}}\right)\right\} .
\end{aligned}
$$

Diferenciando $\mathrm{L}(\boldsymbol{\beta})$ com respeito a $\beta_{i j}$ obtém-se

$$
\begin{aligned}
\frac{\partial \mathrm{L}(\boldsymbol{\beta})}{\partial \beta_{i j}} & =\sum_{i=1}^{n}\left\{z_{1 i} \frac{\partial \eta_{1 i}}{\partial \beta_{1 j}}-\frac{\left(z_{0 i}+z_{1 i}+z_{2 i}\right)}{1+e^{\eta_{1 i}}+e^{\eta_{2 i}}} e^{\eta_{1 i}} \frac{\partial \eta_{1 i}}{\partial \beta_{1 j}}\right\}=\sum_{i=1}^{n}\left\{z_{1 i} x_{i j}-\left(z_{0 i}+z_{1 i}+z_{2 i}\right) \pi_{1 i} x_{i j}\right\} \\
& =\sum_{i=1}^{n}\left(z_{1 i} x_{i j}-\pi_{1 i} x_{1 j}\right)=\sum_{i=1}^{n} x_{i j}\left(z_{1 i}-\pi_{1 i}\right) .
\end{aligned}
$$

Em forma matricial tem-se que

$$
\mathbf{U}_{1}(\boldsymbol{\beta})=\frac{\partial \mathrm{L}(\boldsymbol{\beta})}{\partial \boldsymbol{\beta}_{1}}=\mathbf{X}^{T}\left(\mathbf{z}_{1}-\boldsymbol{\pi}_{1}\right)
$$

em que $\mathbf{X}$ é uma matriz $n \times p$ de linhas $\mathbf{x}_{i}^{T}, \quad \mathbf{z}_{1}=\left(z_{11}, \ldots, z_{1 n}\right)^{T}$ e $\boldsymbol{\pi}_{1}=\left(\pi_{11}, \ldots, \pi_{1 n}\right)^{T}$. De forma similar chega-se à função escore para $\boldsymbol{\beta}_{2}$

$$
\mathbf{U}_{2}(\boldsymbol{\beta})=\frac{\partial \mathrm{L}(\boldsymbol{\beta})}{\partial \boldsymbol{\beta}_{2}}=\mathbf{X}^{T}\left(\mathbf{z}_{2}-\boldsymbol{\pi}_{2}\right)
$$


em que $\mathbf{X}$ é uma matriz $n \times p$ de linhas $\mathbf{x}_{i}^{T}, \quad \mathbf{z}_{2}=\left(z_{21}, \ldots, z_{2 n}\right)^{T}$ e $\boldsymbol{\pi}_{2}=\left(\pi_{21}, \ldots, \pi_{2 n}\right)^{T}$.

Portanto, a função escore para $\boldsymbol{\beta}$ pode ser expressa na forma matricial

$$
\mathbf{U}(\boldsymbol{\beta})=\left[\begin{array}{c}
\mathbf{U}_{1}(\boldsymbol{\beta}) \\
\mathbf{U}_{2}(\boldsymbol{\beta})
\end{array}\right]=\left[\begin{array}{c}
\mathbf{X}^{T}\left(\mathbf{z}_{1}-\boldsymbol{\pi}_{1}\right) \\
\mathbf{X}^{T}\left(\mathbf{z}_{2}-\boldsymbol{\pi}_{2}\right)
\end{array}\right]
$$

que é uma matriz $2 p \times 1$. Ou ainda, se definirmos a matriz $\mathbf{S}$ e os vetores $\mathbf{z}$ e $\boldsymbol{\pi}$ abaixo

$$
\mathbf{S}=\left[\begin{array}{cc}
\mathbf{X} & \mathbf{0} \\
\mathbf{0} & \mathbf{X}
\end{array}\right], \quad \mathbf{z}=\left[\begin{array}{c}
\mathbf{z}_{1} \\
\mathbf{z}_{2}
\end{array}\right] \quad \text { e } \quad \boldsymbol{\pi}=\left[\begin{array}{c}
\boldsymbol{\pi}_{1} \\
\boldsymbol{\pi}_{2}
\end{array}\right]
$$

então poderemos reescrever (1.6) na forma matricial simplificada

$$
\mathbf{U}(\boldsymbol{\beta})=\mathbf{S}^{T}(\mathbf{z}-\boldsymbol{\pi})
$$

\subsection{Matriz de Informação}

Vamos agora obter a matriz de informação de Fisher para $\boldsymbol{\beta}$. Antes porém precisamos obter as matrizes Hessianas para $\boldsymbol{\beta}_{1}$ e $\boldsymbol{\beta}_{2}$.

Diferenciando-se $\mathrm{L}(\boldsymbol{\beta})$ com respeito a $\beta_{1 j}$ e $\beta_{1 l}$ obtém-se

$$
\frac{\partial^{2} \mathrm{~L}(\boldsymbol{\beta})}{\partial \beta_{1 j} \partial \boldsymbol{\beta}_{1 l}}=-\sum_{i=1}^{n} \frac{e^{\eta_{1 i}}\left(1+e^{\eta_{2 i}}\right)}{\left(1+e^{\eta_{1 i}}+e^{\eta_{2 i}}\right)^{2}} x_{i j} x_{i l}=-\sum_{i=1}^{n} \pi_{1 i}\left(1-\pi_{1 j}\right) x_{i j} x_{i l} .
$$

De forma similar a derivada de $\mathrm{L}(\boldsymbol{\beta})$ com respeito a $\beta_{2 j}$ e $\beta_{2 l}$ fica dada por

$$
\frac{\partial^{2} \mathrm{~L}(\boldsymbol{\beta})}{\partial \beta_{2 j} \partial \boldsymbol{\beta}_{2 l}}=-\sum_{i=1}^{n} \pi_{2 i}\left(1-\pi_{2 j}\right) x_{i j} x_{i l}
$$

A derivada cruzada de $\mathrm{L}(\boldsymbol{\beta})$ com respeito a $\beta_{1 j}$ e $\beta_{2 l}$ fica dada por

$$
\frac{\partial^{2} \mathrm{~L}(\boldsymbol{\beta})}{\partial \beta_{1 j} \partial \boldsymbol{\beta}_{2 l}}=\sum_{i=1}^{n} \frac{e^{\eta_{1 i}} e^{\eta_{2 i}}}{\left(1+e^{\eta_{1 i}}+e^{\left.\eta_{2 i}\right)^{2}}\right.} x_{i j} x_{i l}=\sum_{i=1}^{n} \pi_{1 i} \pi_{2 i} x_{i j} x_{i l} .
$$

$\mathrm{Na}$ forma matricial as matrizes Hessianas assumem, respectivamente, as formas

$$
\frac{\partial^{2} \mathrm{~L}(\boldsymbol{\beta})}{\partial \beta_{1} \partial \boldsymbol{\beta}_{1}^{T}}=-\mathbf{X}^{T} \mathbf{V}_{1} \mathbf{X}, \quad \frac{\partial^{2} \mathrm{~L}(\boldsymbol{\beta})}{\partial \beta_{2} \partial \boldsymbol{\beta}_{2}^{T}}=-\mathbf{X}^{T} \mathbf{V}_{2} \mathbf{X} \quad \text { e } \quad \frac{\partial^{2} \mathrm{~L}(\boldsymbol{\beta})}{\partial \beta_{1} \partial \boldsymbol{\beta}_{2}^{T}}=-\mathbf{X}^{T} \mathbf{V}_{3} \mathbf{X}
$$

em que 


$$
\begin{aligned}
& \mathbf{V}_{1}=\operatorname{diag}\left\{\pi_{11}\left(1-\pi_{11}\right), \ldots, \pi_{1 n}\left(1-\pi_{1 n}\right)\right\} \\
& \mathbf{V}_{2}=\operatorname{diag}\left\{\pi_{21}\left(1-\pi_{21}\right), \ldots, \pi_{2 n}\left(1-\pi_{2 n}\right)\right\} \\
& \mathbf{V}_{3}=\operatorname{diag}\left\{-\pi_{11} \pi_{21}, \ldots,-\pi_{1 n} \pi_{2 n}\right\}
\end{aligned}
$$

Portanto, a matriz de informação de Fisher para $\boldsymbol{\beta}$ assume a forma

$$
\mathbf{K}(\boldsymbol{\beta})=E\left\{-\frac{\partial^{2} \mathrm{~L}(\boldsymbol{\beta})}{\partial \boldsymbol{\beta} \partial \boldsymbol{\beta}^{T}}\right\}=\left[\begin{array}{ll}
\mathbf{X}^{T} \mathbf{V}_{1} \mathbf{X} & \mathbf{X}^{T} \mathbf{V}_{3} \mathbf{X} \\
\mathbf{X}^{T} \mathbf{V}_{3} \mathbf{X} & \mathbf{X}^{T} \mathbf{V}_{2} \mathbf{X}
\end{array}\right]
$$

que é uma matriz não singular $2 p \times 2 p$. Podemos reexpressar (1.8) na forma abaixo

$$
\mathbf{K}(\boldsymbol{\beta})=\mathbf{S}^{T} \mathbf{V S}
$$

em que

$$
\mathbf{V}=\left[\begin{array}{ll}
\mathbf{V}_{1} & \mathbf{V}_{3} \\
\mathbf{V}_{3} & \mathbf{V}_{2}
\end{array}\right]
$$

\subsection{Processo Iterativo}

Para estimar os parâmetros $\boldsymbol{\beta}_{1}$ e $\boldsymbol{\beta}_{2}$ pelo metódo de máxima verossimilhança deve-se fazer $\mathbf{U}_{1}(\boldsymbol{\beta})=0$ e $\mathbf{U}_{2}(\boldsymbol{\beta})=0$. Devido a concavidade da função $\mathrm{L}(\boldsymbol{\beta})$ em $\boldsymbol{\beta}$ tem-se a garantia de que as raízes acima levam às estimativas de máxima verossimilhança $\hat{\boldsymbol{\beta}}_{1}$ e $\hat{\boldsymbol{\beta}}_{2}$. Como $\mathbf{U}_{1}(\boldsymbol{\beta})=0$ e $\mathbf{U}_{2}(\boldsymbol{\beta})=0$ são equações não-lineraes que em geral não levam a soluções explícitas para $\hat{\boldsymbol{\beta}}_{1}$ e $\hat{\boldsymbol{\beta}}_{2}$, será utilizado o método de Newton-Raphson para resolver as equações acima. Chega-se facilmente ao seguinte processo iterativo:

$$
\boldsymbol{\beta}^{(m+1)}=\boldsymbol{\beta}^{(m)}+\mathbf{K}^{-1}\left(\boldsymbol{\beta}^{(m)}\right) \mathbf{U}\left(\boldsymbol{\beta}^{(m)}\right),
$$

que é o mesmo que

$$
\left(\begin{array}{l}
\boldsymbol{\beta}_{1}^{(m+1)} \\
\boldsymbol{\beta}_{2}^{(m+1)}
\end{array}\right)=\left(\begin{array}{l}
\boldsymbol{\beta}_{1}^{(m)} \\
\boldsymbol{\beta}_{2}^{(m)}
\end{array}\right)+\left[\begin{array}{ll}
\mathbf{X}^{T} \mathbf{V}_{1}^{(m)} \mathbf{X} & \mathbf{X}^{T} \mathbf{V}_{3}^{(m)} \mathbf{X} \\
\mathbf{X}^{T} \mathbf{V}_{3}^{(m)} \mathbf{X} & \mathbf{X}^{T} \mathbf{V}_{2}^{(m)} \mathbf{X}
\end{array}\right]^{-1} \times\left[\begin{array}{l}
\mathbf{X}^{T}\left(\mathbf{z}_{1}-\boldsymbol{\pi}_{1}^{(m)}\right) \\
\mathbf{X}^{T}\left(\mathbf{z}_{2}-\boldsymbol{\pi}_{2}^{(m)}\right)
\end{array}\right]
$$

com $m=0,1,2 \ldots$

Similarmente à regressão logística binomial, pode-se iniciar o processo iterativo (1.9) em $\boldsymbol{\beta}_{1}^{(0)}=\boldsymbol{\beta}_{2}^{(0)}=0$, que é equivalente a iniciá-lo em $\boldsymbol{\pi}_{1}^{(0)}=\boldsymbol{\pi}_{2}^{(0)}=(1 / 2, \ldots, 1 / 2)^{T}$. 


\subsection{Função Desvio}

Para definir a função desvio de forma análoga aos modelos lineares generalizados (vide, por exemplo, McCullagh e Nelder, 1989; Paula, 2003) considere inicialmente o caso de k grupos em que $Y_{i j}, i=1, \ldots, k$ e $j=1, \ldots, n_{i}$, segue distribuição trinomial com probabilidades $\pi_{0 i}=P\left(Y_{i j}=0\right), \pi_{1 i}=P\left(Y_{i j}=1\right)$ e $\pi_{2 i}=P\left(Y_{i j}=2\right)$. Logo, para cada $Y_{i j}$ pode-se definir a variável aleatória $\mathbf{Z}_{i j}=\left(Z_{0 i j}, Z_{1 i j}, Z_{2 i j}\right)^{T}$ com as mesmas atribuições de $\mathbf{Z}$ definida anteriormente.

Portanto, a função de probabilidade de $\left(\mathbf{Z}_{11}, \ldots, \mathbf{Z}_{1 n_{1}}, \ldots, \mathbf{Z}_{k 1}, \ldots, \mathbf{Z}_{k n_{k}}\right)$ pode ser expressa na forma

$$
g\left(\mathbf{z}_{11}, \ldots, \mathbf{z}_{k n_{k}} ; \boldsymbol{\pi}_{1}, \ldots, \boldsymbol{\pi}_{k}\right)=\prod_{i=1}^{k} \prod_{j=1}^{n_{i}} \pi_{0 i}^{z_{0 i j}} \pi_{1 i}^{z_{1 i j}} \pi_{2 i}^{z_{2 i j}}
$$

em que $\mathbf{z}_{i j}=\left(z_{0 i j}, z_{1 i j}, z_{2 i j}\right)^{T}$ e $\boldsymbol{\pi}_{i}=\left(\pi_{0 i}, \pi_{1 i}, \pi_{2 i}\right)^{T}$ com as restrições $\pi_{0 i}+\pi_{1 i}+\pi_{2 i}=1 \mathrm{e}$ $\sum_{j=1}^{n_{i}}\left(z_{0 i j}+z_{1 i j}+z_{2 i j}\right)=n_{i}$. Tem-se ainda que $n=n_{1}+n_{2}+\ldots+n_{k}$.

Chamando $a_{0 i}=\sum_{j=1}^{n_{i}} z_{0 i j}, \quad a_{1 i}=\sum_{j=1}^{n_{i}} z_{1 i j} \quad e \quad a_{2 i}=\sum_{j=1}^{n_{i}} z_{2 i j}$, a função de probabilidade (1.10) fica dada por

$$
g\left(\mathbf{z}_{11}, \ldots, \mathbf{z}_{k n_{k}} ; \boldsymbol{\pi}_{1}, \ldots, \boldsymbol{\pi}_{k}\right)=\prod_{i=1}^{k} \pi_{0 i}^{a_{0 i}} \pi_{1 i}^{a_{1 i}} \pi_{2 i}^{a_{2 i}}
$$

O logaritmo da função de verossimilhança de $\boldsymbol{\pi}=\left(\boldsymbol{\pi}_{1}^{T}, \ldots, \boldsymbol{\pi}_{k}^{T}\right)^{T}$ fica dado por

$$
\mathrm{L}(\boldsymbol{\pi})=\log \left\{g\left(\mathbf{z}_{11}, \ldots, \mathbf{z}_{k n_{k}} ; \boldsymbol{\pi}_{1}, \ldots, \boldsymbol{\pi}_{k}\right)\right\}=\sum_{i=1}^{k}\left\{a_{0 i} \log \pi_{0 i}+a_{1 i} \log \pi_{1 i}+a_{2 i} \log \pi_{2 i}\right\}
$$

Derivando $\mathrm{L}(\boldsymbol{\pi})$ com respeito a $\pi_{0 i}, \pi_{1 i}, \pi_{2 i}$ e igualando essas derivadas a zero, chega-se às estimativas de máxima verossimilhança saturadas, dadas por

$$
\tilde{\pi}_{0 i}=\frac{a_{0 i}}{n_{i}}, \quad \tilde{\pi}_{1 i}=\frac{a_{1 i}}{n_{i}} \text { e } \tilde{\pi}_{2 i}=\frac{a_{2 i}}{n_{i}}
$$


Note que $a_{0 i}+a_{1 i}+a_{2 i}=n_{i}$ e $\tilde{\pi}_{0 i}+\tilde{\pi}_{1 i}+\tilde{\pi}_{2 i}=1$. Considere a parte sistemática do modelo dada por (1.1) e (1.2) cujas estimativas de máxima verossimilhança para $\pi_{0 i}, \pi_{1 i}$ e $\pi_{2 i}$ serão, respectivamente, dadas por $\hat{\pi}_{0 i}, \hat{\pi}_{1 i}$ e $\hat{\pi}_{2 i}$. A função desvio que pode ser interpretada como uma distância entre o logaritmo da função de verossimilhança do modelo saturado e o logaritmo da função de verossimilhança do modelo sob investigação fica dada por

$D(\hat{\boldsymbol{\pi}} ; \mathbf{z})=2\{\mathrm{~L}(\tilde{\boldsymbol{\pi}})-\mathrm{L}(\hat{\boldsymbol{\pi}})\}=2 \sum_{i=1}^{k}\left\{a_{0 i} \times \log \left(\tilde{\boldsymbol{\pi}}_{0 i} / \hat{\boldsymbol{\pi}}_{0 i}\right)+a_{1 i} \times \log \left(\tilde{\boldsymbol{\pi}}_{1 i} / \hat{\boldsymbol{\pi}}_{1 i}\right)+a_{2 i} \times \log \left(\tilde{\boldsymbol{\pi}}_{2 i} / \hat{\boldsymbol{\pi}}_{2 i}\right)\right\}$.

Se ocorrer

$$
\left(a_{0 i}=n_{i}, a_{1 i}=0, a_{2 i}=0\right), \quad\left(a_{0 i}=0, a_{1 i}=n_{i}, a_{2 i}=0\right) \quad \text { ou } \quad\left(a_{0 i}=0, a_{1 i}=0, a_{2 i}=n_{i}\right)
$$

então o i-ésimo termo de $D(\hat{\boldsymbol{\pi}} ; \mathbf{z})$ fica, respectivamente, dado por $-2 n_{i} \log \left(\hat{\pi}_{0 i}\right), \quad-2 n_{i} \log \left(\hat{\pi}_{1 i}\right)$ ou $-2 n_{i} \log \left(\hat{\pi}_{2 i}\right)$.

Supondo que $\frac{n_{i}}{n} \rightarrow \gamma_{i}>0, \forall_{i}$, conforme $n \rightarrow \infty$, tem-se que $D(\hat{\boldsymbol{\pi}} ; \mathbf{z})$ segue sob a hipótese de que o modelo sob investigação é correto, uma distribuição qui-quadrado com $(2 k-2 p)$ graus de liberdade. Logo, rejeita-se o modelo postulado se $D(\hat{\boldsymbol{\pi}} ; \mathbf{z}) \geq \chi_{(2 k-2 p)}^{2}(1-$ $\alpha)$, sendo $\chi_{(2 k-2 p)}^{2}(1-\alpha)$ o percentil $(1-\alpha)$ de uma qui-quadrado com $(2 k-2 p)$ graus de liberdade. Esse resultado assintótico não continua valendo quando $k \rightarrow \infty, n_{i} \pi_{0 i}\left(1-\pi_{0 i}\right)$ e $n_{i} \pi_{1 i}\left(1-\pi_{1 i}\right)$ permanecem limitados. Neste caso pode-se utilizar a estatística de Hosmer e Lemeshow (1989) que será descrita na próxima seção.

A função escore para $\boldsymbol{\beta}$ neste caso de $\mathrm{k}$ grupos fica dada por

$$
\mathbf{U}(\boldsymbol{\beta})=\left[\begin{array}{c}
\mathbf{U}_{1}(\boldsymbol{\beta}) \\
\mathbf{U}_{2}(\boldsymbol{\beta})
\end{array}\right]=\left[\begin{array}{l}
\mathbf{X}^{T}\left(\mathbf{a}_{1}-\boldsymbol{\mu}_{1}\right) \\
\mathbf{X}^{T}\left(\mathbf{a}_{2}-\boldsymbol{\mu}_{2}\right)
\end{array}\right]
$$

sendo que $\mathbf{a}_{1}=\left(a_{11}, a_{12}, \ldots, a_{1 k}\right)^{T}, \mathbf{a}_{2}=\left(a_{21}, a_{22}, \ldots, a_{2 k}\right)^{T}, \boldsymbol{\mu}_{1}=\left(n_{1} \pi_{11}, n_{1} \pi_{12} \ldots, n_{1} \pi_{1 k}\right)^{T}$ e $\boldsymbol{\mu}_{2}=\left(n_{2} \pi_{21}, n_{2} \pi_{22} \ldots, n_{2} \pi_{2 k}\right)^{T}$. 
A matriz de informação de Fisher para $\boldsymbol{\beta}$ assume a mesma forma dada em (1.8), porém com as mesmas matrizes $\mathbf{V}_{1}, \mathbf{V}_{2}$ e $\mathbf{V}_{3}$ dadas por

$$
\begin{aligned}
& \mathbf{V}_{1}=\operatorname{diag}\left\{n_{1} \pi_{11}\left(1-\pi_{11}\right), \ldots, n_{k} \pi_{1 k}\left(1-\pi_{1 k}\right)\right\}, \\
& \mathbf{V}_{2}=\operatorname{diag}\left\{n_{1} \pi_{21}\left(1-\pi_{21}\right), \ldots, n_{k} \pi_{2 k}\left(1-\pi_{2 k}\right)\right\} \\
& \mathbf{V}_{3}=\operatorname{diag}\left\{-n_{1} \pi_{11} \pi_{21}, \ldots,-n_{k} \pi_{1 k} \pi_{2 k}\right\} .
\end{aligned}
$$

O processo iterativo para estimar $\boldsymbol{\beta}$ assume no caso de réplicas a forma dada abaixo

$$
\boldsymbol{\beta}^{(m+1)}=\boldsymbol{\beta}^{(m)}+\left[\mathbf{S}^{T} \mathbf{V}^{(m)} \mathbf{S}\right]^{-1} \mathbf{S}^{T}\left(\mathbf{a}-\boldsymbol{\mu}^{(m)}\right),
$$

em que

$$
\mathbf{V}=\left[\begin{array}{ll}
\mathbf{V}_{1} & \mathbf{V}_{3} \\
\mathbf{V}_{3} & \mathbf{V}_{2}
\end{array}\right], \quad \mathbf{a}=\left(\begin{array}{c}
\mathbf{a}_{1} \\
\mathbf{a}_{2}
\end{array}\right) \quad \text { e } \boldsymbol{\mu}=\left(\begin{array}{l}
\boldsymbol{\mu}_{1} \\
\boldsymbol{\mu}_{2}
\end{array}\right)
$$

com $\mathrm{m}=0,1,2, \ldots$

\subsection{Estatística de Hosmer e Lemeshow}

O teste qui-quadrado proposto por Hosmer e Lemeshow (1989) para avaliar a qualidade do ajuste de um modelo logístico binomial com dados não agrupados pode ser estendido com pequenas adaptações para cada um dos sub-modelos logísticos que compõe o modelo trinomial. Assim, para o sub-modelo que explica as probabilidades $P\left(Y_{i}=1\right)=\pi_{1 i}$ através da relação dada abaixo

$$
\pi_{1 i}=\frac{e^{\eta_{1 i}}}{1+e^{\eta_{1 i}}+e^{\eta_{2 i}}}
$$

podemos desenvolver uma estatística tipo qui-quadrado para avaliar a qualidade do ajuste.

Sejam as probabilidades ajustadas nessa categoria denotadas por $\hat{\pi}_{11}, \ldots, \hat{\pi}_{1 n}$. Essas probabilidades serão colocadas em ordem não decrescente, tais que

$$
\hat{\pi}_{1(1)} \leq \hat{\pi}_{1(2)} \leq \ldots \leq \hat{\pi}_{1(n)}
$$


em que $\hat{\pi}_{1(1)}$, por exemplo, denota a menor probabilidade ajustada dessa categoria e $\hat{\pi}_{1(n)}$ a maior probabilidade ajustada. Supor ainda que os $n$ indivíduos são agrupados em $g$ grupos definidos conforme as probabilidades ajustadas acima, de modo que o primeiro grupo tenha $n_{11}$ elementos e assim por diante até o último grupo com $n_{1 g}$ elementos. Para o l-ésimo grupo formado tem-se o valor observado de $O_{1 l}$ sucessos, ou seja, $O_{1 l}$ indivíduos (dentre os $n_{1 l}$ indivíduos) com resposta $(\mathrm{Y}=1)$. O valor esperado para esse grupo será dado por $n_{1 l} \bar{\pi}_{1 l}$, em que $\bar{\pi}_{1 l}$ denota o valor médio das probabilidades estimadas do grupo. Assim, a estatística de Hosmer-Lemeshow para esse sub-modelo ajustado fica dada por

$$
\mathrm{C}_{1}^{H L}=\sum_{l=1}^{g} \frac{\left(O_{1 l}-n_{1 l} \bar{\pi}_{1 l}\right)^{2}}{n_{1 l} \bar{\pi}_{1 l}\left(1-\bar{\pi}_{1 l}\right)} .
$$

A sugestão é comparar $\mathrm{C}_{1}^{H L}$ com os percentis da distribuição qui-quadrado com $(g-2)$ graus de liberdade, desde que $n_{1 l}, \forall_{l}$, não seja pequeno. O ajuste não será adequado se

$$
\mathrm{C}_{1}^{H L} \geq \chi_{(g-2)}^{2}(1-\alpha)
$$

em que $\chi_{(g-2)}^{2}(1-\alpha)$ denota o percentil $(1-\alpha)$ de uma distribuição qui-quadrado com $(g-2)$ graus de liberdade. A sugestão é usar aproximadamente $g=10$ grupos.

Similarmente, para o sub-modelo que explica as probabilidades $P\left(Y_{i}=2\right)=\pi_{2 i}$ através da relação dada abaixo

$$
\pi_{2 i}=\frac{e^{\eta_{2 i}}}{1+e^{\eta_{1 i}}+e^{\eta_{2 i}}}
$$

podemos definir a estatística

$$
\mathrm{C}_{2}^{H L}=\sum_{l=1}^{g} \frac{\left(O_{2 l}-n_{2 l} \bar{\pi}_{2 l}\right)^{2}}{n_{2 l} \bar{\pi}_{2 l}\left(1-\bar{\pi}_{2 l}\right)} .
$$

O modelo logístico trinomial estará bem ajustado se as duas estatísticas $\mathrm{C}_{1}^{H L}$ e $\mathrm{C}_{2}^{H L}$ apresentarem valores não significativos.

\subsection{Resultados Inferenciais}

Supondo que $\frac{n_{i}}{n} \rightarrow \gamma_{i}>0, \forall_{i}$, conforme $n \rightarrow \infty$, o estimador de máxima verossimilhança $\hat{\boldsymbol{\beta}}$ segue uma distribuição normal $(2 p)$-variada de média $\boldsymbol{\beta}$ e matriz de variância-covariância 
$\hat{\mathbf{K}}^{-1}(\boldsymbol{\beta})$.

Para testar hipóteses suponha, por exemplo, que o interesse seja testar $H_{0}: \mathbf{C} \boldsymbol{\beta}=0$ contra $H_{1}: \mathbf{C} \boldsymbol{\beta} \neq 0$, em que $\mathbf{C}$ é uma matriz $r \times(2 p)$ de posto completo $(r<2 p)$. Denote por $\hat{\boldsymbol{\beta}}^{0}$ a estimativa de máxima verossimilhança sob $H_{0}$. Assim, a estatística da razão de verossimilhanças para testar $H_{0}$ contra $H_{1}$ fica dada por

$$
\begin{aligned}
\xi_{R V} & =2\left\{\mathrm{~L}(\hat{\boldsymbol{\pi}})-\mathrm{L}\left(\hat{\boldsymbol{\pi}}^{0}\right)\right\} \\
& =2 \sum_{i=1}^{k}\left\{a_{0 i} \times \log \left(\hat{\boldsymbol{\pi}}_{0 i} / \hat{\boldsymbol{\pi}}_{0 i}^{0}\right)+a_{1 i} \times \log \left(\hat{\boldsymbol{\pi}}_{1 i} / \hat{\boldsymbol{\pi}}_{1 i}^{0}\right)+a_{2 i} \times \log \left(\hat{\boldsymbol{\pi}}_{2 i} / \hat{\boldsymbol{\pi}}_{2 i}^{0}\right)\right\}
\end{aligned}
$$

sendo $\hat{\boldsymbol{\pi}}^{0}$ a estimativa de $\boldsymbol{\pi}$ sob $H_{0}$.

Para $\frac{n_{i}}{n} \rightarrow \gamma_{i}>0, \forall_{i}$, conforme $n \rightarrow \infty$, a estatística $\xi_{R V}$ segue sob $H_{0}$ uma distribuição qui-quadrado com $(2 p-r)$ graus de liberdade. É interessante notar que (1.12) pode ser expressa como a diferença entre dois desvios

$$
\xi_{R V}=D\left(\hat{\boldsymbol{\pi}}^{0} ; \mathbf{z}\right)-D(\hat{\boldsymbol{\pi}} ; \mathbf{z})
$$

Uma situação de interesse prático seria testar a homogeneidade dos coeficientes dos dois sub-modelos logísticos (1.1) e (1.2), ou seja, testar $H_{0}: \beta_{1 j}=\beta_{2 j} \quad(j=1,2, \ldots, p)$ contra $H_{1}: \beta_{1 j} \neq \beta_{2 j}$, para algum $\mathbf{j}$. Neste caso a matriz $\mathbf{C}$ assume a forma

$$
\mathbf{C}=\left(\begin{array}{rrrrrrrr}
1 & 0 & \ldots & 0 & -1 & 0 & \ldots & 0 \\
0 & 1 & \ldots & 0 & 0 & -1 & \ldots & 0 \\
& & \ldots & & & & & \\
0 & 0 & \ldots & 1 & 0 & 0 & \ldots & -1
\end{array}\right)
$$

ou seja, $\mathbf{C}$ é uma matriz $(r \times 2 p)$ sendo $r=p$. Sob $H_{0}$, a estatística $\xi_{R V}$ segue uma distribuição qui-quadrado com $p$ graus de liberdade. 


\subsection{Exemplos}

\subsubsection{Exemplo 1}

Vamos considerar para uma primeira análise os dados de um estudo conduzido (vide

Hosmer e Lemeshow, 1989) para avaliar fatores associados com conhecimento, atitude e comportamento de mulheres em relação a mamografia. Neste estudo temos o seguinte:

Variável resposta

Experiência: experiência com mamografia, codificada como

0: nunca realizou o exame de mamografia (referência),

1: submeteu-se ao exame de mamografia nos últimos 12 meses e

2: submeteu-se ao exame de mamografia há mais de um ano.

Variáveis explicativas

Sintoma: você não precisa de mamografia a menos que desenvolva os sintomas?

1: concordo totalmente (referência),

2: concordo,

3: discordo e

4: discordo totalmente.

Benefício: benefício percebido (soma de 5 variáveis com escala de 4 pontos cada, ou seja, assume valores de 5 a 20). Um valor baixo indica que a mulher tem forte concordância com os benefícios da mamografia.

Histórico: você tem mãe ou irmã com hitórico de câncer de mama?

0: não e

1: sim. 
Autoexame: alguém ensinou a você como se auto-examinar?

0: não e

1: sim.

Detectar: você acha provável que a mamografia possa detectar novos casos de câncer de mama?

1: pouco provável (referência),

2: provável e

3: muito provável.

\section{Modelagem}

Denote por $Y_{i}$ a experiência da i-ésima mulher entrevistada $(i=1,2, \ldots, n)$, em que $n=412$. Será assumido que $Y_{i}$ segue distribuição trinomial $\left(Y_{i}=0\right.$ nunca realizou o exame, $Y_{i}=1$ realizou o exame nos últimos 12 meses e $Y_{i}=2$ realizou o exame há mais de um ano) com probabilidades dadas por $\pi_{0 i}, \pi_{1 i}$ e $\pi_{2 i}$, respectivamente.

\section{VARIÁvel ExPlicativa BinÁRIA}

A título de ilustração vamos propor uma modelagem considerando apenas a variável explicativa binária Histórico. A parte sistemática do modelo fica dada por

$$
\log \left\{\frac{\pi_{1 i}}{\pi_{0 i}}\right\}=\beta_{10}+\beta_{1} \times \text { Historico }_{i} \quad \text { e } \quad \log \left\{\frac{\pi_{2 i}}{\pi_{0 i}}\right\}=\beta_{20}+\beta_{2} \times \text { Historico }_{i} .
$$

O subescrito nas razões de chances abaixo indica que a resposta está sendo comparada ao nível de referência $(Y=0)$. Isto é, a razão de chances da resposta $j$ versus a resposta 0 para os valores da variável explicativa $X=a$ versus $X=b$ é

$$
\psi_{j}(a, b)=\frac{P(Y=j \mid X=a) / P(Y=0 \mid X=a)}{P(Y=j \mid X=b) / P(Y=0 \mid X=b)} .
$$


Tabela 1.1

\begin{tabular}{ccccc}
\multicolumn{5}{c}{ Classificação cruzada experiência versus histórico familiar. } \\
\hline \hline Experiência & Histórico & familiar & & \\
com mamografia & 0 & 1 & Total & $\hat{\psi}_{j}$ \\
\hline 0 & 220 & 14 & 234 & 1,00 \\
1 & 85 & 19 & 104 & 3,51 \\
2 & 63 & 11 & 74 & 2,74 \\
\hline Total & 368 & 44 & 412 & \\
\hline \hline
\end{tabular}

Usando a notação adotada, as duas razões de chances estimadas da Tabela 1.1 são:

$$
\hat{\psi}_{1}=\frac{19 \times 220}{85 \times 14}=3,51 \quad \text { e } \quad \hat{\psi}_{2}=\frac{11 \times 220}{63 \times 14}=2,74 .
$$

As estatísticas acima informam que a chance de uma mulher com histórico de câncer na família ter se submetido ao exame nos últimos 12 meses é 3,51 vezes a de uma mulher que ainda não teve esta experiência, além disso a chance de ter realizado o exame há mais de um ano para uma mulher que já teve a experiência na família é 2,74 vezes a de uma mulher que nunca teve a experiência familiar. O resultado do ajuste do modelo trinomial aos dados (vide apêndice A) é descrito na Tabela 1.2.

\section{Tabela 1.2}

Resultados do modelo de regressão logística ajustado à variável histórico.

\begin{tabular}{clrrrrr}
\hline \hline \multirow{2}{*}{ Logito } & Variável & Coeficiente & Erro padrão & Estatística & \multicolumn{2}{c}{ Nível } \\
Estimado & Estimado & de Wald & Descritivo & $\hat{\psi}_{j}$ \\
\hline \multirow{2}{*}{2} & Historico & 1,2564 & 0,3747 & 11,2448 & 0,0008 & 3,51 \\
& Intercepto & $-0,9510$ & 0,1277 & 55,4474 & 0,0001 & \\
& Historico & 1,0093 & 0,4275 & 5,5744 & 0,0182 & 2,74 \\
& Intercepto & $-1,2505$ & 0,1429 & 76,5842 & 0,0001 & \\
\hline \hline
\end{tabular}

$-2 \times \log ($ verossimilhança $)=792,340$

Valores ajustados de $\hat{\psi}_{j}$ podem ser também obtidos exponenciando o coeficiente angular estimado (coluna $\hat{\psi}_{j}$ na Tabela 1.2). As estimativas para $\psi_{j}$ das duas tabelas coincidem. Isso ocorre devido à propriedade de invariância da estimativa de máxima verossimilhança. O mesmo modelo está sendo ajustado em ambos os casos com parametrizações diferentes. 
Da Tabela 1.2 temos as seguintes equações estimadas:

$\log \left\{\frac{\hat{\pi}_{1 i}}{\hat{\pi}_{0 i}}\right\}=-0,951+1,256 \times$ Historico $_{i}$ e $\log \left\{\frac{\hat{\pi}_{2 i}}{\hat{\pi}_{0 i}}\right\}=-1,251+1,009 \times$ Historico $_{i}$.

Usando os resultados da Tabela 1.2 podemos construir intervalos de confiança aproximados para os coeficientes, que exponenciando nos levam a intervalos de confiança aproximados para as razões de chances. Por exemplo, um intervalo de confiança aproximado de 95\% para a razão de chances de $Y=1$ versus $Y=0$ fica dado por

$$
\exp (1,256 \pm 1,96 \times 0,375)=[1,69 ; 7,32] .
$$

O logaritmo da razão de chances para $Y=2$ versus $Y=1$ pode ser obtido da diferença entre os dois coeficientes angulares estimados no modelo de regressão logística correspondente. Ilustrando com os resultados da Tabela 1.2 obtemos

$$
\log \left(\frac{19 \times 63}{11 \times 85}\right)=1,256-1,009=0,247
$$

mostrando que ao comparar mulheres que já fizeram o exame, a chance de ter realizado nos últimos 12 meses é maior (1,28 vezes) que ter realizado a mais de um ano quando a mulher já teve a experiência na família do que quando nunca teve histórico de câncer familiar.

Para uma indicação preliminar da importância da variável, vamos utilizar o teste da razão de verossimilhanças (1.12) para avaliar a significância dos coeficientes:

$$
\xi_{R V}=2\left\{\mathrm{~L}(\hat{\boldsymbol{\pi}})-\mathrm{L}\left(\hat{\boldsymbol{\pi}}^{0}\right)\right\}=805,198-792,340=12,8581
$$

Como foi visto em (1.12), $\xi_{R V}$ segue sob $H_{0}$ (os coeficientes são nulos) uma distribuição qui-quadrado com $(2 p-r)$ graus de liberdade, quando $n$ é grande. Para esse caso, temos 2 graus de liberdade o que produz um nível descritivo de 0,0016. Assim, do ponto de vista inferencial, o fato da mulher ter um histórico de câncer na família está fortemente associado com a decisão de submeter-se a um exame de mamografia. 


\section{VÁRIVEl Explicativa Policotômica}

Para uma variável explicativa policotômica expandimos o número de razões de chances para incluir comparações de cada nível ao nível de referência para cada logito possível. Para ilustrar, vamos considerar a variável Detectar que será substituída por duas variáveis binárias Detectar ${ }_{2}(=1$ provável, $=0$ caso contrário $)$ e Detecta 3 ( $=1$ muito provável, $=0$ caso contrário).

A parte sistemática do modelo fica dado por

$$
\begin{aligned}
& \log \left\{\frac{\pi_{1 i}}{\pi_{0 i}}\right\}=\beta_{10}+\beta_{11} \times \operatorname{detectar}_{2 i}+\beta_{12} \times \operatorname{detectar}_{3 i} \\
& \mathrm{e} \\
& \log \left\{\frac{\pi_{2 i}}{\pi_{0 i}}\right\}=\beta_{20}+\beta_{21} \times \text { detectar }_{2 i}+\beta_{22} \times \text { detectar }_{3 i} .
\end{aligned}
$$

Tabela 1.3

Classificação cruzada experiência versus detectar.

\begin{tabular}{ccccc}
\hline \hline Experiência & \multicolumn{4}{c}{ Detectar } \\
com mamografia & 1 & 2 & 3 & Total \\
\hline 0 & 13 & 77 & 144 & 234 \\
1 & 1 & 12 & 91 & 104 \\
2 & 4 & 16 & 54 & 74 \\
\hline Total & 18 & 105 & 289 & 412 \\
\hline \hline
\end{tabular}

As estimativas das razões de chances obtidas da Tabela 1.3 são dadas abaixo

$$
\begin{array}{ll}
\hat{\psi}_{1}(2,1)=\frac{12 \times 13}{77 \times 1}=2,03, & \hat{\psi}_{1}(3,1)=\frac{91 \times 13}{144 \times 1}=8,22, \\
\hat{\psi}_{2}(2,1)=\frac{16 \times 13}{77 \times 4}=0,68 \quad \text { e } \quad \hat{\psi}_{2}(3,1)=\frac{54 \times 13}{144 \times 4}=1,22 .
\end{array}
$$

Por exemplo, a chance de uma mulher ter feito o exame nos últimos 12 meses é 2,03 vezes a de nunca ter feito quando a mesma considera provável que um exame possa detectar o problema frente a mulheres que acham pouco provável a detecção. A chance cresce para 8,22 vezes se a mulher acreditar que seja muito provável que um mamograma 
detecte o problema. Para os casos em que os exames foram realizados há mais de um ano as chances mudam pouco. Os resultados do ajuste do modelo estão resumidos na Tabela 1.4 .

Tabela 1.4

Resultados do modelo de regressão logística ajustado à variável detectar.

\begin{tabular}{clrrrrr}
\hline \hline \multirow{2}{*}{ Logito } & & Coeficiente & Erro padrão & Estatística & \multicolumn{2}{c}{ Nível } \\
Eariável & Estimado & Estimado & de Wald & Descritivo & $\hat{\psi}_{j}$ \\
\hline 1 & Detectar $_{2}$ & 0,706 & 1,083 & 0,425 & 0,515 & 2,02 \\
& Detectar $_{3}$ & 2,106 & 1,046 & 4,051 & 0,044 & 8,22 \\
& Intercepto $_{2} 2$ & $-2,565$ & 1,038 & 6,109 & 0,013 & \\
& Detectar $_{2}$ & $-0,393$ & 0,634 & 0,383 & 0,536 & 0,68 \\
& Detectar $_{3}$ & 0,198 & 0,594 & 0.111 & 0,739 & 1,22 \\
& Intercepto $^{2}$ & $-1,179$ & 0,572 & 4,249 & 0,039 & \\
\hline \hline
\end{tabular}

As equações estimadas são dadas abaixo

$$
\begin{aligned}
& \log \left\{\frac{\hat{\pi}_{1 i}}{\hat{\pi}_{0 i}}\right\}=-2,565+0,706 \times \text { detectar }_{2 i}+2,106 \times \operatorname{detectar}_{3 i} \\
& \log \left\{\frac{\hat{\pi}_{2 i}}{\hat{\pi}_{0 i}}\right\}=-1,179-0,393 \times \text { detectar }_{2 i}+0,198 \times \text { detectar }_{3 i} .
\end{aligned}
$$

Usaremos a razão de verossimilhanças para avaliar a significância da variável Detectar. O valor da estatística é $\xi_{R V}=805,198-778,401=26,797$ com 4 graus de liberdade, produzindo um nível descrítivo menor que 0,001. Assim, podemos concluir que a crença da mulher na capacidade da mamografia detectar um novo caso de câncer de mama está associado com sua decisão de fazer um exame de mamografia.

Examinando-se os coeficientes estimados e suas estatística de Wald, notamos que a associação é mais forte quando comparamos mulheres que fizeram o exame nos últimos 12 meses com mulheres que nunca se submeteram ao exame, e quando comparando respostas pouco provável a muito provável. Todos os demais coeficientes são não significantes. 


\section{EstratéGia PARA SELEÇÃo DE MODELOS}

Em principio, as estratégias e métodos para seleção de modelos com variável resposta trinomial são os mesmos do caso binomial. Por exemplo, a metodologia para seleção de variáveis stepwise funciona bem no caso binomial, entretanto para o caso trinomial a analogia não é direta, uma vez que trabalhamos com duas funções simultaneamente. Para o nosso caso vamos ultilizar a estatística de Wald e o teste da razão de verossimilhanças para a seleção do modelo. Um outro método de seleção que poderia ser estendido para o modelo logístico trinomial é o método de Akaike que no caso consistiria em encontrar o modelo com $2 p$ parâmetros tal que

$$
\mathrm{AIC}=\frac{1}{2} \mathrm{D}_{2 p}+2 p
$$

seja mínimo, em que $D_{2 p}$ denote o desvio do modelo ajustado.

Na análise dos dados do estudo de mamografia existem apenas cinco variáveis independentes e 412 observações (vide Apêndice A). A variável Sintoma está codificada em quatro níveis, no processo de construção do modelo vamos modelá-la em três variáveis binárias, usando o primeiro nível concordo totalmente como referência. A variável Detectar está codificada em três níveis, será modelada usando duas variáveis binárias, usando o primeiro nível pouco provável como referência. Apesar dessas duas variáveis serem ordinais é razoável codificá-las em variáveis binárias ao invés de tratá-las como contínuas, pois dessa forma os coeficientes para as variáveis binárias podem fornecer uma interpretação direta de cada nível da varável Detectar. As variávies Historico e Autoexame são binárias, o primeiro nível será usado como referência nos dois casos. Inicialmente, vamos tratar a variável Beneficio como se fosse contínua e linear nos logitos. Os resultados para o ajuste considerando todas as variáveis (apenas efeitos principais) são apresentados na Tabela 1.5 
Tabela $\mathbf{1 . 5}$

Resultados do modelo de regressão logística ajustado aos dados do Apêndice A.

\begin{tabular}{|c|c|c|c|c|c|}
\hline Logito & Variável & $\begin{array}{c}\text { Coeficiente } \\
\text { Estimado }\end{array}$ & $\begin{array}{c}\text { Erro padrão } \\
\text { Estimado }\end{array}$ & $\begin{array}{c}\text { Estatística } \\
\text { de Wald }\end{array}$ & $\begin{array}{c}\text { Nível } \\
\text { Descritívo }\end{array}$ \\
\hline \multirow[t]{9}{*}{1} & Sintoma $_{2}$ & 0,1098 & 0,9226 & 0,0142 & 0,9052 \\
\hline & Sintoma $_{3}$ & 1,9242 & 0,7774 & 6,1258 & 0,0133 \\
\hline & Sintoma $_{4}$ & 2,4565 & 0,7752 & 10,0422 & 0,0015 \\
\hline & Beneficio & $-0,2194$ & 0,0755 & 8,4444 & 0,0037 \\
\hline & Historico & 1,3662 & 0,4375 & 9,7512 & 0,0018 \\
\hline & Autoexame & 1,2916 & 0,5299 & 5,9416 & 0,0148 \\
\hline & Detectar $_{2}$ & 0,0161 & 1,1615 & 0,0002 & 0,9889 \\
\hline & Detectar $_{3}$ & 0,9032 & 1,1264 & 0,6429 & 0,4226 \\
\hline & Intercepto & $-2,9973$ & 1,5389 & 3,7937 & 0,0514 \\
\hline \multirow[t]{9}{*}{2} & Sintoma $_{2}$ & $-0,2901$ & 0,6441 & 0,2029 & 0,6524 \\
\hline & Sintoma $_{3}$ & 0,8173 & 0,5398 & 2,2925 & 0,1300 \\
\hline & Sintoma $_{4}$ & 1,1322 & 0,5477 & 4,2739 & 0,0387 \\
\hline & Beneficio & $-0,1482$ & 0,0764 & 3,7662 & 0,0523 \\
\hline & Historico & 1,0654 & 0,4594 & 5,3786 & 0,0204 \\
\hline & Autoexame & 1,0521 & 0,5150 & 4,1739 & 0,0411 \\
\hline & Detectar $_{2}$ & $-0,9244$ & 0,7138 & 1,6774 & 0,1953 \\
\hline & Detectar $_{3}$ & $-0,6906$ & 0,6871 & 1,0100 & 0,3149 \\
\hline & Intercepto & $-0,9861$ & 1,1118 & 0,7865 & 0,3751 \\
\hline
\end{tabular}

De acordo com a estatística de Wald, os dois coeficientes estimados para a variável binária Sintoma 2 , que estima o logaritmo da razão de chances para resposta concordo versus o valor de referência concordo totalmente, sugere que essas duas categorias são similares. O sinal e magnitude dos coeficientes estimados para as variáveis Sintoma 3 e Sintoma Su- $^{-}$ gere que as respostas discordo e discordo totalmente diferem da referência concordo totalmente e são similares dentro de cada uma das duas funções logito. Este tipo de estrutura nos coeficientes estimados sugere que devemos dicotomizar Sintoma em apenas dois níveis, $0=$ concordo totalmente ou concordo e $1=$ discordo ou discordo totalmente, e em seguida reajustar o modelo. Essa nova variável dicotômica será rotulada como SintomaD. O novo ajuste considerando essa nova codificação é resumido na Tabela 1.6. 
Tabela 1.6

Resultados do modelo de regreesão logística ajustado aos dados do Apêndice A.

\begin{tabular}{clcccc}
\hline \hline \multirow{2}{*}{ Logito } & Variável & $\begin{array}{c}\text { Coeficiente } \\
\text { Estimado }\end{array}$ & $\begin{array}{c}\text { Erro padrão } \\
\text { Estimado }\end{array}$ & $\begin{array}{c}\text { Estatística } \\
\text { de Wald }\end{array}$ & $\begin{array}{c}\text { Nível } \\
\text { Descritívo }\end{array}$ \\
\hline 1 & SintomaD & 2,0950 & 0,4573 & 20,9842 & 0,0001 \\
& Beneficio & $-0,2510$ & 0,0729 & 11,8454 & 0,0006 \\
& Historico & 1,2933 & 0,4335 & 8,8990 & 0,0029 \\
& Autoexame & 1,2439 & 0,5263 & 5,5863 & 0,0181 \\
& Detectar $_{2}$ & 0,0893 & 1,1606 & 0,0059 & 0,9386 \\
& Detectar $_{3}$ & 0,9719 & 1,1259 & 0,7451 & 0,3880 \\
& Intercepto $_{2} 2$ & $-2,7024$ & 1,4341 & 3,5510 & 0,0595 \\
& SintomaD & 1,1214 & 0,3572 & 9,8552 & 0,0017 \\
& Beneficio & $-0,1681$ & 0,0742 & 5,1366 & 0,0234 \\
& Historico & 1,0140 & 0,4538 & 4,9932 & 0,0254 \\
& Autoexame & 1,0286 & 0,5140 & 4,0049 & 0,0454 \\
& Detectar $_{2}$ & $-0,9022$ & 0,7146 & 1,5936 & 0,2068 \\
& Detectar $_{3}$ & $-0,6698$ & 0,6876 & 0,9490 & 0,3300 \\
& Intercepto & $-0,9987$ & 1,0720 & 0,8680 & 0,3515 \\
\hline \hline
\end{tabular}

$-2 \times \log ($ verossimilhança $)=697,496$

Resta agora avaliar a contribuição ao modelo da variável Detectar. Na Tabela 1.6 observamos que nenhuma das estatísticas de Wald para as variáveis binárias Detectar são significativas marginalmente, porém acreditava-se que no total, a variável ainda poderia ser significativa e também uma confundidora para alguma outra variável. Para avaliar sua significância e seu papel como confundidora ajustamos o modelo excluindo a variável Detectar. O valor da estatística do teste da razão de verossimilhanças para a significância da variável Detectar é 8,54 com 4 graus de liberdade, produzindo um nível descritivo de 0,074. Nota-se Tabela 1.7 nas variáveis restantes no modelo uma sensível mudança no coeficiente estimado da variável SintomaD no primeiro logito. A mudança foi de cerca de $8 \%$ no valor do coeficiente estimado, indicando que a variável Detectar exerce um papel de confundidora da associação da variável SintomaD com a variável resposta. Devido a esse papel e sua proximidade à significância estatística, decidimos mantê-la no modelo. 
As estimativas para os modelos sem a variável Detectar estão resumidas na Tabela 1.7.

Tabela 1.7

Resultado do modelo de regresão excluindo a variável Detectar.

\begin{tabular}{clcccc}
\hline \hline Logito & Variável & $\begin{array}{c}\text { Coeficiente } \\
\text { Estimado }\end{array}$ & $\begin{array}{c}\text { Erro padrão } \\
\text { Estimado }\end{array}$ & $\begin{array}{c}\text { Estatística } \\
\text { de Wald }\end{array}$ & $\begin{array}{c}\text { Nível } \\
\text { Descritívo }\end{array}$ \\
\hline \multirow{2}{*}{1} & SintomaD & 2,2302 & 0,4519 & 24,3538 & 0,0001 \\
& Beneficio & $-0,2825$ & 0,0713 & 15,6819 & 0,0001 \\
& Historico & 1,2966 & 0,4293 & 9,1223 & 0,0025 \\
& Autoexame & 1,2209 & 0,5210 & 5,4910 & 0,0191 \\
& Intercepto & $-1,7885$ & 0,8470 & 4,4584 & 0,0347 \\
2 & SintomaD & 1,1531 & 0,3514 & 10,7697 & 0,0010 \\
& Beneficio & $-0,1578$ & 0,0712 & 4,9144 & 0,0266 \\
& Historico & 1,0613 & 0,4527 & 5,4969 & 0,0191 \\
& Autoexame & 0,9604 & 0,5072 & 3,5853 & 0,0583 \\
& Intercepto & $-1,7421$ & 0,8087 & 4,6410 & 0,0312 \\
\hline \hline
\end{tabular}

$-2 \times \log ($ verossimilhança $)=706,038$

O próximo passo no desenvolvimento do modelo é avaliar a necessidade de incluir os termos de interação de $1^{a}$ ordem no modelo. Neste modelo cada par de variável cria uma interação biologicamente plausível.

Nenhuma das 10 possíveis interações contribuiram significativamente para o modelo. Assim, as equações estimadas para o modelo selecionado, são as seguintes:

$$
\begin{aligned}
\log \left\{\frac{\hat{\pi}_{1 i}}{\left.\hat{\pi}_{0 i}\right\}=}\right. & -2,7024+2,0950 \times \text { SintomaD }_{i}-0,2510 \times \text { Beneficio }_{i}+ \\
& 1,2933 \times \text { Historico }_{i}+1,2439 \times \text { Autoexame }_{i}+0,0893 \times \text { Detectar }_{2 i}+ \\
& 0,9719 \times \text { Detectar }_{3 i}
\end{aligned}
$$

e

$$
\begin{aligned}
\log \left\{\frac{\hat{\pi}_{2 i}}{\left.\hat{\pi}_{0 i}\right\}=}\right. & -0,9987+1,1214 \times \text { SintomaD }_{i}-0,1681 \times \text { Beneficio }_{i}+ \\
& 1,0140 \times \text { Historico }_{i}+1,0286 \times \text { Autoexame }_{i}-0,9022 \times \text { Detectar }_{2 i}- \\
& 0,6698 \times \text { Detectar }_{3 i} .
\end{aligned}
$$




\subsubsection{Exemplo 2}

Este estudo foi realizado (vide Forthofer and Lehnen, 1981 e Apêndice B) para investigar a associação entre resultado no teste respiratório, hábito de fumar e idade. A amostra consistiu de 2219 trabalhadores da indústria de Houston, Texas, durante os anos de 1974 e 1975. Neste estudo são consideradas as seguintes variáveis.

Variável resposta

Resultado: resultado obtido no teste respiratório:

1: normal (referência),

2: indefinido e

3: anormal.

Variáveis explicativas

Status: condição atual do pesquisado:

1: nunca fumou (referência),

2: ex-fumante e

3: fumante.

Idade: idade do pesquisado:

1: menos de 40 anos e

2: 40 a 59 anos.

\section{Modelagem}

Denote por $Y_{i}$ o resultado do i-ésimo exame respiratório $(i=1,2, \ldots, n)$, em que $n=$ 2219. Novamente assumiremos que $Y_{i}$ segue distribuição trinomial $\left(Y_{i}=1\right.$ normal, $Y_{i}=2$ indefinido e $Y_{i}=3$ anormal) com probabilidades dadas por $\pi_{o i}, \pi_{1 i}$ e $\pi_{2 i}$, respectivamente. 
Vamos iniciar nossa modelagem considerando primeiramente a variável explicativa idade.

A parte sistemática do modelo fica dada por

$$
\begin{aligned}
& \log \left\{\frac{\pi_{1 i}}{\pi_{0 i}}\right\}=\beta_{10}+\beta_{1} \times \text { idade }_{i} \\
& \log \left\{\frac{\pi_{2 i}}{\pi_{0 i}}\right\}=\beta_{20}+\beta_{2} \times \text { idade }_{i} .
\end{aligned}
$$

Os coeficientes e razões de chances estimadas estão dadas na Tabela 1.9

\begin{tabular}{|c|c|c|c|c|c|c|}
\hline Logito & Variável & $\begin{array}{r}\text { Coeficiente } \\
\text { Estimado }\end{array}$ & $\begin{array}{c}\text { Erro padrão } \\
\text { Estimado }\end{array}$ & $\begin{array}{r}\text { Estatística } \\
\text { de Wald }\end{array}$ & $\begin{array}{c}\text { Nível } \\
\text { Descritivo }\end{array}$ & $\hat{\psi}_{j}$ \\
\hline \multirow[t]{2}{*}{1} & Idade & 0,6199 & 0,1685 & 13,5315 & 0,0002 & 1,86 \\
\hline & Intercepto & $-3,3673$ & 0,2504 & 180,7722 & 0,0000 & \\
\hline \multirow[t]{2}{*}{2} & Idade & 1,4447 & 0,2820 & 26,2446 & 0,0000 & 4,24 \\
\hline & Intercepto & $-5,6802$ & 0,4738 & 143,7485 & 0,0000 & \\
\hline
\end{tabular}

Tabela 1.9

Resultados do modelo de regressão logística ajustado à variável idade.

As razões de chances acima informam que a chance de um indivíduo com idade entre 40 e 59 anos ter o resultado de seu teste respiratório anormal é 4,2 vezes a de um resultado normal. Além disso, a chance de ter o resultado indefinido para o seu teste respiratório é 1,9 vezes a de um resultado normal.

A razão de chances para $Y=3$ versus $Y=2$ pode ser obtida da razão entre as duas razões de chances estimadas no modelo de regressão logística correspondente. Da Tabela 1.9 obtemos $\hat{\psi}_{3}=\hat{\psi}_{2} / \hat{\psi}_{1}=4,241 / 1,859=2,28$. Mostrando que ao compararmos pessoas com resultados não normais no teste respiratório, a chance de ter resultado anormal é maior (2,28 vezes) do que ter um resultado indefinido quando o pesquisado faz parte do grupo na faixa etária de 40 a 59 anos.

Para uma indicação preliminar da importância da variável, vamos avaliar a significância dos coeficientes. O teste da razão de verossimilhanças nos fornece um nível descritivo 
menor que 0,0001, dando indicação de que esta variável é significativa quando avaliada isoladamente.

Vamos agora considerar a variável explicativa status. A parte sistemática do modelo fica dada por

$$
\begin{aligned}
& \log \left\{\frac{\pi_{1 i}}{\pi_{0 i}}\right\}=\beta_{10}+\beta_{1} \times \text { status }_{2_{i}}+\beta_{2} \times \text { status }_{3_{i}} \\
& \mathrm{e} \\
& \log \left\{\frac{\pi_{2 i}}{\pi_{0 i}}\right\}=\beta_{20}+\beta_{1} \times \text { status }_{2_{i}}+\beta_{2} \times \text { status }_{3_{i}} .
\end{aligned}
$$

A Tabela 1.10 exibe os coeficientes e razões de chances estimadas

Tabela 1.10

Resultados do modelo de regressão logística ajustado à variável status.

\begin{tabular}{clrcrrr}
\hline \hline \multirow{2}{*}{ Logito } & Coeficiente & Erro padrão & Estatística & \multicolumn{2}{c}{ Nível } & \\
\hline \multirow{2}{*}{1} & Variável & Estimado & Estimado & de Wald & Descritivo & $\hat{\psi}$ \\
& status $_{2}$ & 0,9093 & 0,2552 & 12,6912 & 0,0004 & 2,48 \\
& status $_{3}$ & 0,8747 & 0,2132 & 16,8364 & 0,0001 & 2,40 \\
& Intercepto $_{2}^{2}$ & $-3,1740$ & 0,1833 & 299,7644 & 0,0001 & \\
& status $_{2}$ & 1,1446 & 0,4972 & 5,3003 & 0,0213 & 3,14 \\
& status $_{3}$ & 1,4677 & 0,4142 & 12,5536 & 0,0004 & 4,34 \\
& Intercepto & $-4,6621$ & 0,3797 & 150,7219 & 0,0001 & \\
\hline \hline
\end{tabular}

Analisando as razões de chances podemos afirmar que a chance de um indivíduo ter o resultado de seu teste respiratório anormal é 4,3 vezes a de um resultado normal se o mesmo for fumante. Esta chance decresce para 3,1 se o indivíduo for ex-fumante. Avaliando a chance de ter o resultado do teste indefinido contra normal, podemos afirmar que é praticamente indiferente para um ex-fumante ou fumante, sendo que a chance é aproximadamente $150 \%$ maior. Da Tabela 1.10 obtemos:

$$
\hat{\psi}_{5}=\hat{\psi}_{22} / \hat{\psi}_{12}=3,141 / 2,483=1,27 \quad \text { e } \quad \hat{\psi}_{6}=\hat{\psi}_{23} / \hat{\psi}_{13}=4,339 / 2,398=1,81 \text {. }
$$


As estatísticas acima mostram que ao compararmos pessoas com resultados não normais no teste respiratório verifica-se que, a chance do resultado ser anormal é $81 \%$ maior se o indivíduo é fumante e $27 \%$ maior se é um ex-fumante.

Para verificar a importância da variável, vamos avaliar a significância dos coeficientes. O teste da razão de verossimilhanças nos fornece um nível descritivo menor que 0,0001, indicando que a variável status está associada ao resultado no teste respiratório.

Consideramos agora as duas variáveis explicativas conjuntamente, usando apenas os efeitos principais, uma vez que a interação idade $\times$ status mostrou-se não significativa. A parte sistemática do modelo fica dada por

$$
\begin{gathered}
\log \left\{\frac{\pi_{1 i}}{\pi_{0 i}}\right\}=\beta_{10}+\beta_{1} \times \text { idade }_{i}+\beta_{2} \times \text { status }_{2_{i}}+\beta_{3} \times \text { status }_{3_{i}} \\
\log \left\{\frac{\pi_{2 i}}{\pi_{0 i}}\right\}=\beta_{20}++\beta_{1} \times \text { idade }_{i}+\beta_{2} \times \text { status }_{2_{i}}+\beta_{3} \times \text { status }_{3_{i}} .
\end{gathered}
$$

As estimativas para o modelo acima são apresentados na Tabela 1.11.

\begin{tabular}{|c|c|c|c|c|c|}
\hline Logito & Variável & $\begin{array}{r}\text { Coeficiente } \\
\text { Estimado }\end{array}$ & $\begin{array}{c}\text { Erro padrão } \\
\text { Estimado }\end{array}$ & $\begin{array}{r}\text { Estatística } \\
\text { de Wald }\end{array}$ & $\begin{array}{c}\text { Nível } \\
\text { Descritivo }\end{array}$ \\
\hline \multirow[t]{4}{*}{1} & Idade & 0,5380 & 0,1713 & 9,8597 & 0,0017 \\
\hline & status $_{2}$ & 0,7881 & 0,2588 & 9,2723 & 0,0023 \\
\hline & status $_{3}$ & 0,8319 & 0,2140 & 15,1194 & 0,0001 \\
\hline & Intercepto & $-3,8508$ & 0,2894 & 177,0264 & 0,0001 \\
\hline \multirow[t]{4}{*}{2} & Idade & 1,3786 & 0,2855 & 23,3130 & 0,0001 \\
\hline & status $_{2}$ & 0,8221 & 0,5031 & 2,6702 & 0,1022 \\
\hline & status $_{3}$ & 1,3463 & 0,4164 & 10,4549 & 0,0012 \\
\hline & Intercepto & $-6,5231$ & 0,5745 & 128,9185 & 0,0001 \\
\hline
\end{tabular}

\section{Tabela 1.11}

Resultados do modelo de regressão logística ajustado aos dados do apêndice $B$.

Ao considerar as duas variáveis explicativas temos que o teste de razão de verossimilhanças produz um valor de 68,8545 com 6 graus de liberdade, o que fornece um nível descritívo 
menor que 0,0001. Portanto, podemos afirmar que essas duas variáveis são altamente significativas, ou seja,tanto idade como status de fumo estão associadas ao resultado de um teste respiratório.

Assim, os sub-modelos ajustados ficam dados por:

$$
\begin{aligned}
& \log \left\{\frac{\hat{\pi}_{1 i}}{\hat{\pi}_{0 i}}\right\}=-3,8508+0,5380 \times \text { idade }_{i}+0,7881 \times \text { status }_{2_{i}}+0,8319 \times \text { status }_{3_{i}} \\
& \log \left\{\frac{\hat{\pi}_{2 i}}{\hat{\pi}_{0 i}}\right\}=-6,5231+1,3786 \times \text { idade }_{i}+0,8221 \times \text { status }_{2_{i}}+1,3463 \times \text { status }_{3_{i}} .
\end{aligned}
$$

Nota-se pelos coeficientes estimados acima que a variável binária idade é mais importante nas estimativas da probabilidade de $\pi_{2}$ de resultado anormal do que na estimativa da probabilidade $\pi_{1}$ de resultado indiferente. O mesmo ocorre para a variável binária status 3 enquanto a outra variável binária status ${ }_{2}$ tem a mesma importância na estimativa das duas probabilidades. 
Capítulo 2 


\section{Capítulo 2}

\section{Métodos de Diagnóstico}

\subsection{Introdução}

Uma etapa importante na análise de um ajuste de regressão é a verificação de possíveis afastamentos das suposições feitas para o modelo, especialmente para a parte aleatória e para a parte sistemática do modelo, bem como a existência de observações extremas com alguma interferência desproporcional nos resultados do ajuste. Tal etapa, conhecida como análise de diagnóstico, tem longa data, e iniciou-se com a análise de resíduos para detectar a presença de pontos extremos e avaliar a adequação da distribuição proposta para a variável resposta. Uma referência importante nesse tópico é o artigo de Cox e Snell (1968) em que é apresentada uma forma bastante geral de definir resíduos, usada até os dias atuais.

Belsley, Kuh e Welsch (1980) e Cook e Weisberg (1982) discutem a padronização de resíduos para o caso normal linear. Pregibon (1981) propõe o componente do desvio como resíduo na classe dos modelos lineares generalizados (MLGs) e sugere uma padronização que é mais tarde comprovada matematicamente por McCullagh (1987) que usa as aproximações propostas por Cox e Snell (1968). Nesse mesmo trabalho McCullagh apresenta uma outra forma de padronização para o componente do desvio em que procura corrigir os efeitos de assimetria e curtose. Atkinson (1981) propõe a construção por simulação 
de Monte Carlo de uma banda de confiança para os resíduos de regressão normal linear, a qual denominou envelopes, e que permite uma melhor comparação entre os resíduos e os percentis da distribuição normal padrão. Williams $(1984,1987)$ discute, com base em estudos de simulação de Monte Carlo, a aproximação da forma padronizada proposta por Pregibon (1981) encontrando fortes evidências de concordância entre a distribuição empírica do componente do desvio padronizado e a distribuição normal padrão para vários MLGs. Williams (1987) também discute a construção de envelopes em MLGs. Davison e Gigli (1989) estendem a proposta de Cox e Snell (1968) e definem uma forma geral de padronização para o componente do desvio para distribuições contínuas, mesmo quando a função de distribuição acumulada não é expressa em forma fechada. Fahrmeir e Tutz (1994) estendem o trabalho de McCullagh (1987) para modelos mais gerais. Paula (1995) apresenta uma forma padronizada para o componente do desvio em MLGs com parâmetros restritos na forma de desigualdades lineares $\mathbf{C} \boldsymbol{\beta} \geq 0$ e verifica através de simulação, forte concordância na maioria dos modelos estudados entre a distribuição empírica do resíduo padronizado e a distribuição normal padrão, generalizando os resultados de Williams para parâmetros restritos. De Souza e Paula (2002) usam o método proposto por Davison e Gigli (1989) a fim de obterem uma forma padronizada para o componente do desvio em modelos de regressão von Mises, os quais têm sido aplicados na análise de dados circulares. A construção de envelopes com o resíduo proposto é também discutida no trabalho.

Neste capítulo serão apresentadas algumas técnicas usuais de diagnóstico que têm sido largamente aplicadas no modelo logístico binomial e que podem ser diretamente estendidas para o modelo logístico trinomial.

\subsection{Pontos de Alavanca}

Um tópico importante de análise de diagnóstico é a detecção de observações influentes, isto é, pontos que exercem um peso desproporcional nas estimativas dos parâmetros do modelo. Durante a década de 70 surgiram várias propostas relacionadas com a influência 
das observações nas estimativas dos coeficientes do modelo normal linear. O estudo da diagonal principal da matriz de projeção $\mathbf{H}=\mathbf{X}\left(\mathbf{X}^{T} \mathbf{X}\right)^{-1} \mathbf{X}^{T}$ apresentada por Hoaglin e Welsch (1978), em que $\mathbf{X}$ denota a matriz modelo, motivou a definição de pontos de alavanca que receberam este nome por terem peso desproporcional no próprio valor ajustado. Esses pontos em geral são remotos no subespaço gerado pelas colunas da matriz X, ou seja, têm um perfil diferente dos demais pontos no que tange aos valores das variáveis explicativas. Dependendo da localização, tais pontos podem exercer forte influência nas estimativas dos coeficientes de regressão. Extensões da definição de pontos de alavanca para modelos normais não-lineares são dadas em St. Laurent e Cook (1992). Recentemente, Wei, Hu e Fung (1998) generalizaram a definição de pontos de alavanca para modelos bastante gerais cuja variável resposta seja contínua. Nessa generalização incluem-se outros métodos de estimação além de máxima verossimilhança, e outros enfoques tais como enfoque Bayesiano. Paula (1999) discute pontos de alavanca em modelos de regressão com parâmetros restritos na forma $\mathbf{C} \boldsymbol{\beta} \geq 0$, com extensões para os MLGs. Em modelos de regressão em que a resposta é contínua pontos de alavanca são aqueles cuja derivada $\frac{\partial \hat{y}_{i}}{\partial y_{i}}$ assume um valor desproporcional, isto é, uma perturbação infinitesimal em $y_{i}$ causa uma grande variação em $\hat{y}_{i}$. Expressões gerais para essa derivada podem ser encontradas em Wei, Hu e Fung (1998). Todavia, quando a resposta é discreta, como é o caso da distribuição trinomial, não faz sentido o cálculo da derivada. Nesses casos, outros conceitos de pontos de alavanca são utilizados. Em particular, no caso da regressão logística binomial usa-se os elementos da diagonal principal da matriz de projeção correspondente ao modelo linear com solução de mínimios quadrados dada por

$$
\hat{\boldsymbol{\beta}}=(\mathbf{X} \hat{\mathbf{V}} \mathbf{X})^{-1} \mathbf{X}^{T} \hat{\mathbf{V}} \mathbf{z}
$$

em que $\hat{\mathbf{V}}=\operatorname{diag}\left\{\mathrm{n}_{1} \pi_{1}\left(1-\pi_{1}\right), \ldots, \mathrm{n}_{\mathrm{k}} \pi_{\mathrm{k}}\left(1-\pi_{\mathrm{k}}\right)\right\}, \mathbf{X}$ é a matriz modelo, $\mathbf{z}=\left(z_{1}, \ldots, z_{k}\right)^{T}$ com $z_{i}=\hat{n}_{1}+\left(y_{i}-n_{i} \hat{\pi}_{i}\right) / n_{i} \hat{\pi}_{i}\left(1-\hat{\pi}_{i}\right)$. A estimativa (2.1) coincide com a solução de 
máxima verrosimilhança do modelo logístico binomial com parte sistemática dada por

$$
\log \left\{\frac{\pi_{\mathrm{i}}}{1-\pi_{\mathrm{i}}}\right\}=\eta_{\mathrm{i}}=\mathbf{x}_{\mathrm{i}}^{\mathrm{T}} \boldsymbol{\beta} .
$$

Essa matriz de projeção assume a forma

$$
\mathbf{H}=\mathbf{V}^{1 / 2} \mathbf{X}\left(\mathbf{X}^{T} \mathbf{V X}\right)^{-1} \mathbf{X}^{T} \mathbf{V}^{1 / 2}
$$

cujo i-ésimo elemento da diagonal principal fica dado por

$$
h_{i i}=n_{i} \pi_{i}\left(1-\pi_{i}\right) \mathbf{x}_{i}^{T}\left(\mathbf{X}^{T} \mathbf{V X}\right)^{-1} \mathbf{x}_{i} .
$$

Logo, $\hat{h}_{i i}$ que detecta pontos de alavanca no modelo linear correspondente é sugerido também para o modelo logístico binomial, embora não tenha a mesma interpretação nesse modelo. Como a função $\pi_{i}\left(1-\pi_{i}\right)$ é quadrática alguns cuidados devem ser tomados no uso de $h_{i i}$. Hosmer e Lemeshow (1989, Cap. 5) mostram através de estudos numéricos que $h_{i i}$ deve ser pequeno quando $\pi_{i}$ está próximo de zero ou um, deve ser alto para valores de $\pi_{i}$ entre 0,1 e 0,3 ou entre 0,7 e 0,9 e deve ser moderado ou pequeno para valores de $\pi_{i}$ entre 0,3 e 0,7 . Assim, observações com valores para $\hat{h}_{i i}$ maiores do que essas tendências esperadas devem ser olhados com mais atenção. O gráfico de $\hat{h}_{i i}$ contra $\hat{\pi}_{i}$ deve ser usado por ser mais informativo do que o gráfico de $\hat{h}_{i i}$ contra a ordem das observações.

No caso do modelo logístico trinomial tem-se dois conjuntos de valores para $\hat{h}_{i i}$, os quais serão denotados por $\hat{h}_{i i}^{(1)}$ e $\hat{h}_{i i}^{(2)}$. O primeiro desses conjuntos será formado pelos elementos da diagonal principal de

$$
\hat{\mathbf{H}}^{(1)}=\hat{\mathbf{V}}_{1}^{1 / 2} \mathbf{X}\left(\mathbf{X}^{T} \hat{\mathbf{V}}_{1} \mathbf{X}\right)^{-1} \mathbf{X}^{T} \hat{\mathbf{V}}_{1}^{1 / 2}
$$

e assume a forma

$$
\hat{h}_{i i}^{(1)}=n_{1 i} \hat{\pi}_{1 i}\left(1-\hat{\pi}_{1 i}\right) \mathbf{x}_{i}^{T}\left(\mathbf{X}^{T} \hat{\mathbf{V}}_{1} \mathbf{X}\right)^{-1} \mathbf{x}_{i},
$$

em que

$$
\mathbf{V}_{1}=\operatorname{diag}\left\{\mathrm{n}_{1 \mathrm{i}} \pi_{1 \mathrm{i}}\left(1-\pi_{1 \mathrm{i}}\right), \ldots, \mathrm{n}_{\mathrm{ki}} \pi_{\mathrm{ki}}\left(1-\pi_{\mathrm{ki}}\right)\right\}
$$


O outro conjunto deve ser formado pelos elementos

$$
\hat{h}_{i i}^{(2)}=n_{2 i} \hat{\pi}_{2 i}\left(1-\hat{\pi}_{2 i}\right) \mathbf{x}_{i}^{T}\left(\mathbf{X}^{T} \hat{\mathbf{V}}_{1} \mathbf{X}\right)^{-1} \mathbf{x}_{i}
$$

da diagonal principal da matriz

$$
\hat{\mathbf{H}}^{(2)}=\hat{\mathbf{V}}_{2}^{1 / 2} \mathbf{X}\left(\mathbf{X}^{T} \hat{\mathbf{V}}_{2} \mathbf{X}\right)^{-1} \mathbf{X}^{T} \hat{\mathbf{V}}_{2}^{1 / 2} .
$$

Gráficos de $\hat{h}_{i i}^{(1)}$ e $\hat{h}_{i i}^{(2)}$ contra os valores ajustados $\hat{\pi}_{1 i}$ e $\hat{\pi}_{2 i}$, respectivamente, devem ser construídos. Aqueles pontos que se destacarem nos gráficos devem ser olhados com mais atenção. Note que a mesma observação pode ser detectada nos dois gráficos.

Podemos para o modelo logístico trinomial fazer uma analogia com o modelo logístico binomial e tentar encontrar uma matriz de projeção com base na expressão da convergência do processo iterativo para $\hat{\boldsymbol{\beta}}$. Note que na convergência, supondo o caso de réplicas, tem-se o seguinte:

$$
\hat{\boldsymbol{\beta}}=\left(\mathbf{S}^{T} \hat{\mathbf{V}} \mathbf{S}\right)^{-1} \mathbf{S}^{T} \hat{\mathbf{V}} \boldsymbol{\xi}
$$

em que

$$
\boldsymbol{\xi}=\mathbf{S} \hat{\boldsymbol{\beta}}+\hat{\mathbf{V}}^{-1}(\mathbf{a}-\boldsymbol{\mu}) .
$$

Assim, podemos propor a matriz de projeção

$$
\mathbf{M}=\mathbf{S}\left(\mathbf{S}^{T} \mathbf{V S}\right)^{-1} \mathbf{S}^{T} \mathbf{V} .
$$

Note que essa matriz $\mathbf{M}$ tem dimensão $(2 n \times 2 n)$ o que significa dizer que teremos para cada observação duas medidas de alavanca, uma para cada sub-modelo logístico. Para a $i$-ésima observação teremos as seguintes medidas:

$$
\hat{m}_{i i}^{(1)}=\mathbf{s}_{0 i}^{T}\left(\mathbf{S}^{T} \hat{\mathbf{V}} \mathbf{S}\right)^{-1} \hat{\mathbf{V}} \mathbf{s}_{1 i} \quad \text { e } \quad \hat{m}_{i i}^{(2)}=\mathbf{s}_{0 i}^{T}\left(\mathbf{S}^{T} \hat{\mathbf{V}} \mathbf{S}\right)^{-1} \hat{\mathbf{V}} \mathbf{s}_{2 i}
$$

em que

$$
\mathbf{s}_{0 i}^{T}=\left[\mathbf{x}_{i}^{T}, \mathbf{x}_{i}^{T}\right], \quad \mathbf{s}_{1 i}^{T}=\left[\mathbf{x}_{i}^{T} \hat{\pi}_{1 i}\left(1-\hat{\pi}_{1 i}\right),-\mathbf{x}_{i}^{T} \hat{\pi}_{1 i} \hat{\pi}_{2 i}\right] \quad \text { e } \mathbf{s}_{2 i}^{T}=\left[-\mathbf{x}_{i}^{T} \hat{\pi}_{1 i} \pi_{2 i}, \mathbf{x}_{i}^{T} \hat{\pi}_{2 i}\left(1-\hat{\pi}_{2 i}\right)\right] .
$$


Obviamente que os gráficos de $\hat{m}_{i i}^{(1)}$ e $\hat{m}_{i i}^{(2)}$ contra os valores ajustados $\hat{\pi}_{i}$ podem revelar observações influentes nas estimativas $\hat{\boldsymbol{\beta}}_{1}$ e $\hat{\boldsymbol{\beta}}_{2}$, respectivamente. Contudo, como pouco se conhece a respeito do comportamento dessas medidas em relação aos valores ajustados, fica difícil estabelecer critérios para usá-las. É muito razoável esperar que $\hat{m}_{i i}^{(1)}$ e $\hat{m}_{i i}^{(2)}$ sejam mais informativas que $\hat{h}_{i i}^{(1)}$ e $\hat{h}_{i i}^{(2)}$ uma vez que esses últimos são construídos com base nos modelos logísticos marginais enquanto os dois primeiros são construídos para o modelo logístico trinomial.

\subsection{Deleção de Pontos}

A deleção de pontos talvez seja a técnica mais conhecida para avaliar o impacto da retirada de uma observação particular nas estimativas da regressão. A distância de Cook (1977), originalmente desenvolvida para modelos normais lineares, foi rapidamente assimilada e estendida para diversas classes de modelos. Por exemplo, Moolgavkar, Lustbader e Verzon (1984) estendem a metodologia para regressão não-linear com aplicações em estudos emparelhados, Ross (1987) discute a geometria da deleção de casos em regressão nãolinear, Cook, Peña e Weisberg (1988) comparam o afastamento da verossimilhança com medidas tradicionais de deleção de pontos tais como a distância de Cook e o DFFITS $_{i}$, esse último proposto por Belsley, Kuh e Welsch (1980) e Paula e Peres (1988) discutem a deleção de pontos em MLGs com parâmetros restritos na forma $\mathbf{C} \boldsymbol{\beta} \geq 0$. Davison e Tsai (1992) e Cordeiro e Paula (1992) estendem a metodologia para modelos cuja distribuição não pertence à família exponencial de distribuições. Recentemente, Galea, Riquelme e Paula (2000) investigam a metodologia em modelos elípticos multivariados. Referências importantes nesse tópico são, dentre outras, os livros de Cook e Weisberg (1982), Atkinson (1985) e Chatterjee e Hadi (1988). Um problema que pode ocorrer na deleção individual de pontos é o que se denomina masking effect ou seja, deixar de detectar pontos conjuntamente discrepantes. Embora esse procedimento de deleção múltipla de pontos não seja muito popular, provavelmente em virtude do custo computacional envolvido, existem 
vários procedimentos robustos para detecção de pontos discrepantes, muitos dos quais com um custo computacional relativamente baixo (ver, por exemplo, Fung, 1993; Peña e Yohai, 1999). Como em geral esses procedimentos têm sido desenvolvidos para modelos lineares, abre-se uma perspectiva de pesquisas em classes mais abrangentes.

Uma das medidas mais populares de deleção de pontos é conhecida como afastamento da verossimilhança (likelihood displacement) definida abaixo

$$
\mathrm{LD}_{i}=2\left\{\mathrm{~L}(\hat{\boldsymbol{\beta}})-\mathrm{L}\left(\hat{\boldsymbol{\beta}}_{(i)}\right\}\right.
$$

em que $\hat{\boldsymbol{\beta}}_{(i)}$ denota a estimativa de máxima verossimilhança de $\boldsymbol{\beta}=\left(\boldsymbol{\beta}_{1}^{T}, \boldsymbol{\beta}_{2}^{T}\right)^{T}$ sem a i-ésima observação. Uma aproximação quadrática por série de Taylor leva à seguinte aproximação:

$$
\mathrm{LD}_{i}=\left(\hat{\boldsymbol{\beta}}-\hat{\boldsymbol{\beta}}_{(i)}\right)^{T} \mathbf{K}(\hat{\boldsymbol{\beta}})\left(\hat{\boldsymbol{\beta}}-\hat{\boldsymbol{\beta}}_{(i)}\right) .
$$

A fim de obter-se uma forma fechada para $\mathrm{LD}_{i}$ é usual considerar alguma aproximação para $\hat{\boldsymbol{\beta}}_{(i)}$. A aproximação de um passo proposta por Pregibon (1981) no caso de regressão logística binomial poderia ser estendida para o caso trinomial. Assim, a medida de diagnóstico fica dada por

$$
\mathrm{LD}_{i}=\left(\hat{\boldsymbol{\beta}}-\hat{\boldsymbol{\beta}}_{(i)}^{1}\right)^{T} \mathbf{K}(\hat{\boldsymbol{\beta}})\left(\hat{\boldsymbol{\beta}}-\hat{\boldsymbol{\beta}}_{(i)}^{1}\right),
$$

em que $\hat{\boldsymbol{\beta}}_{(i)}^{1}$ denota a aproximação de um passo do processo iterativo para estimar $\boldsymbol{\beta}$ sem a $i$-ésima observação e iniciando em $\hat{\boldsymbol{\beta}}$.

Uma outra possibilidade mais simples seria considerar a aproximação para o afastamento da verossimilhança para cada um dos sub-modelos logísticos que compõe o modelo logístico trinomial. Assim, teremos dois conjuntos de medidas de influência

$$
\operatorname{LD}_{i}^{(1)}=\left(\hat{\boldsymbol{\beta}}_{1}-\hat{\boldsymbol{\beta}}_{1(i)}^{1}\right)^{T} \mathbf{K}_{1}(\hat{\boldsymbol{\beta}})\left(\hat{\boldsymbol{\beta}}_{1}-\hat{\boldsymbol{\beta}}_{1(i)}^{1}\right)
$$




$$
\operatorname{LD}_{i}^{(2)}=\left(\hat{\boldsymbol{\beta}}_{2}-\hat{\boldsymbol{\beta}}_{2(i)}^{1}\right)^{T} \mathbf{K}_{2}(\hat{\boldsymbol{\beta}})\left(\hat{\boldsymbol{\beta}}_{2}-\hat{\boldsymbol{\beta}}_{2(i)}^{1}\right)
$$

em que

$$
\mathbf{K}_{1}(\hat{\boldsymbol{\beta}})=\mathbf{X}^{T} \mathbf{V}_{1} \mathbf{X} \quad \text { e } \quad \mathbf{K}_{2}(\hat{\boldsymbol{\beta}})=\mathbf{X}^{T} \mathbf{V}_{2} \mathbf{X}
$$

A aproximação de um passo proposta por Pregibon (1981) para o modelo logístico binomial com parte sistemática

$$
\log \left\{\frac{\pi_{i}}{1-\pi_{i}}\right\}=\eta_{i}=\mathbf{x}_{i}^{T} \boldsymbol{\beta}
$$

fica dada por

$$
\boldsymbol{\beta}_{(i)}^{1}=\hat{\boldsymbol{\beta}}-\left\{\frac{y_{i}-n_{i} \hat{\pi}_{i}}{1-\hat{h}_{i i}}\right\}\left(\mathbf{X}^{T} \hat{\mathbf{V}} \mathbf{X}\right)^{-1} \mathbf{x}_{i}
$$

em que $\mathbf{V}=\operatorname{diag}\left\{n_{1} \pi_{1}\left(1-\pi_{1}\right), \ldots, n_{k} \pi_{k}\left(1-\pi_{k}\right)\right\}$. Usando (2.4) nas expressões (2.2) e (2.3) chega-se às medidas de influência

$$
\operatorname{LD}_{i}^{(1)}=\left\{\frac{\hat{h}_{i i}^{(1)}}{1-\hat{h}_{i i}^{(1)}}\right\}\left\{t_{S_{i}}^{(1)}\right\}^{2} \quad \text { e } \quad \operatorname{LD}_{i}^{(2)}=\left\{\frac{\hat{h}_{i i}^{(2)}}{1-\hat{h}_{i i}^{(2)}}\right\}\left\{t_{S_{i}}^{(2)}\right\}^{2},
$$

em que

$$
t_{S_{i}}^{(1)}=\frac{\left(a_{1 i}-n_{1 i} \hat{\pi}_{1 i}\right)}{\sqrt{n_{1 i} \hat{\pi}_{1 i}\left(1-\hat{\pi}_{1 i}\right)\left(1-\hat{h}_{i i}^{(1)}\right)}} \quad \text { e } \quad t_{S_{i}}^{(2)}=\frac{\left(a_{2 i}-n_{2 i} \hat{\pi}_{2 i}\right)}{\sqrt{n_{2 i} \hat{\pi}_{2 i}\left(1-\hat{\pi}_{2 i}\right)\left(1-\hat{h}_{i i}^{(2)}\right)}}
$$

são os respectivos resíduos de Pearson padronizados, em que $a_{1 i}$ e $a_{2 i}$ representam o número de sucessos nos níveis 1 e 2 , respectivamente, do $i$-ésimo grupo (vide seção 1.6).

Assim, são sugeridos dois conjuntos de gráficos de diagnóstico. O gráfico de $\operatorname{LD}_{i}^{(1)}$ contra $\hat{\pi}_{1 i}$ e o gráfico de $\operatorname{LD}_{i}^{(2)}$ contra $\hat{\pi}_{2 i}$. Aquelas observações que destoarem com valores muito acima dos demais devem ser retiradas para avaliação do impacto das mesmas nas estimativas. Se a variação for muito desproporcional pode-se dizer que esses pontos são influentes em determinadas estimativas. Porém, deve-se verificar também se a eliminação desses pontos leva a mudanças inferenciais, por exemplo, deixar algum coeficiente não 
significativo. A eliminação definitiva de um ponto somente deve ser feita em último caso após várias tentativas de acomodá-lo no ajuste através, por exemplo, de transformações de variáveis explicativas, inclusão ou eliminação de variáveis explicativas ou interação, ou mesmo mudanças no modelo.

\subsection{Influência Local}

Uma das propostas mais inovadoras na área de diagnóstico em regressão foi apresentada por Cook (1986) que propõe avaliar a influência conjunta das observações sob pequenas mudanças (perturbações) no modelo, ao invés da avaliação pela retirada individual ou conjunta de pontos. Essa metodologia, denominada influência local, teve uma grande receptividade entre os usuários e pesquisadores de regressão, havendo inúmeras publicações no assunto em que se aplica a metodologia em classes particulares de modelos ou em que se propõe extensões da técnica. Por exemplo, na classe de erros normais, Lawrence (1988) investiga a aplicação de influência local em modelos lineares com parâmetros na transformação da resposta, Beckman, Nachtsheim e Cook (1987) apresentam estudos de influência em modelos de análise de variância com efeito misto, Tsai e Wu (1992) investigam influência local em modelos auto-regressivos de $1^{a}$ ordem e modelos heterocedásticos e Paula (1993) aplica influência local em modelos lineares com restrições nos parâmetros na forma de desigualdades lineares. Saindo da classe de erros normais tem-se, por exemplo, o trabalho de Pettitt e Bin Daud (1989) que investigam influência local em modelos de Cox com riscos proporcionais, Escobar e Meeker (1992) adaptam influência local numa classe paramétrica de modelos para análise de sobrevivência, O'Hara Hines, Lawless e Carter (1992), Kim (1995) e Pan, Fang e von Resen (1997) aplicam métodos de influência local em regressão multivariada. Mais recentemente, Galea, Paula e Bolfarine (1997), Liu (2000) e Galea, Paula e Uribe-Opazo (2003) apresentam estudos de

influência local em modelos elípticos lineares, enquanto Kwan e Fung (1998) aplicam a metodologia em análise fatorial, Gu e Fung (1998) em análise de correlação canônica 
e Paula (1996) em modelos próprios de dispersão. Svetliza e Paula (2003) discutem influência local em modelos de regressão com resposta binomial negativa. Esses últimos modelos têm sido muito usados para corrigir problemas de superdispersão, freqüentemente encontrados em modelos com resposta de Poisson. Ortega, Bolfarine e Paula (2003) aplicam influência local em modelos log-gama generalizados com dados censurados. Uma discussão interessante a respeito do uso de influência local é apresentada por Fung e Kwan (1997). Os autores mostram que o afastamento da verossimilhança neste caso é uma medida de influência invariante com mudanças de escala nos dados, fato que não ocorre com outras medidas de influência propostas.

Para formalizar o método de influência local nos modelos logísticos trinomiais considere $\mathrm{L}(\boldsymbol{\beta})$ como sendo o logaritmo da função de verossimilhança do modelo postulado, em que $\boldsymbol{\beta}=\left(\boldsymbol{\beta}_{1}^{T}, \boldsymbol{\beta}_{2}^{T}\right)^{T}$, com $\boldsymbol{\beta}_{1}$ e $\boldsymbol{\beta}_{2}$ denotando os parâmetros da parte sistemática dos dois sub-modelos logísticos formados. Seja $\boldsymbol{\omega}$ um vetor $q \times 1$ de perturbações, restritas a um conjunto aberto $\Omega \subset \Re^{q}$. As perturbações são feitas no logaritmo da verossimilhança de modo que a mesma tome a forma $\mathrm{L}(\boldsymbol{\beta} \mid \boldsymbol{\omega})$. Denotando o vetor de não perturbação por $\boldsymbol{\omega}_{0}$, assume-se que $\mathrm{L}\left(\boldsymbol{\beta} \mid \boldsymbol{\omega}_{0}\right)=\mathrm{L}(\boldsymbol{\beta})$. A fim de verificar-se a influência das perturbações na estimativa de máxima verossimilhança $\hat{\boldsymbol{\beta}}$, considera-se o afastamento da verossimilhança

$$
\mathrm{LD}(\boldsymbol{\omega})=2\left\{\mathrm{~L}(\hat{\boldsymbol{\beta}})-\mathrm{L}\left(\hat{\boldsymbol{\beta}}_{\omega}\right)\right\}
$$

em que $\hat{\boldsymbol{\beta}}_{\omega}$ denota a estimativa de máxima verossimilhança sob o modelo $\mathrm{L}(\boldsymbol{\beta} \mid \boldsymbol{\omega})$.

A idéia de influência local consiste basicamente em estudar o comportamento da função $\operatorname{LD}(\boldsymbol{\omega})$ em torno de $\boldsymbol{\omega}_{0}=0$. O procedimento procura selecionar uma direção unitária $\boldsymbol{\ell},\|\boldsymbol{\ell}\|=1$, e então estudar o gráfico de $\operatorname{LD}\left(\boldsymbol{\omega}_{0}+\mathrm{a} \boldsymbol{\ell}\right)$ contra $a$, em que $a \in \Re$. Esse gráfico é conhecido como linha projetada. Note que, desde que $\operatorname{LD}\left(\boldsymbol{\omega}_{0}\right)=0$, então $\operatorname{LD}\left(\boldsymbol{\omega}_{0}+\mathrm{a} \boldsymbol{\ell}\right)$ tem um mínimo local em $a=0$. Cada linha projetada pode ser caracterizada por uma curvatura normal $\mathrm{C}_{\ell}(\boldsymbol{\beta})$ em torno de $a=0$. Essa curvatura é interpretada como sendo o inverso do raio de melhor círculo ajustado em $a=0$. A sugestão é considerar a direção 
$\boldsymbol{\ell}_{\max }$ que corresponde à maior curvatura $\mathrm{C}_{\ell_{\max }}(\boldsymbol{\beta})$. O gráfico de $\boldsymbol{\ell}_{\max }$ contra a ordem das observações pode revelar quais observações que sob pequenas perturbações exercem uma influência desproporcional em $\operatorname{LD}(\boldsymbol{\omega})$. Cook (1986) mostra que a curvatura normal na direção $\boldsymbol{\ell}$ toma a forma $\mathrm{C}_{\ell}(\boldsymbol{\beta})=2\left|\boldsymbol{\ell}^{\mathrm{T}} \boldsymbol{\Delta}^{\mathrm{T}}(\ddot{\mathbf{L}})^{-1} \boldsymbol{\Delta} \boldsymbol{\ell}\right|$, em que $-\ddot{\mathbf{L}}$ é a matriz de informação observada de Fisher enquanto que $\boldsymbol{\Delta}$ é uma matriz $2 p \times q$ definida no modelo logístico trinomial por

$$
\boldsymbol{\Delta}=\left[\begin{array}{c}
\frac{\partial^{2} L(\beta)}{\partial \beta_{1} \partial \omega^{T}} \\
\frac{\partial^{2} L(\beta)}{\partial \beta_{2} \partial \omega^{T}}
\end{array}\right],
$$

ambas avaliadas em $\boldsymbol{\beta}=\hat{\boldsymbol{\beta}}$ e $\boldsymbol{\omega}=\boldsymbol{\omega}_{0}, i=1, \ldots, p$ e $j=1, \ldots, q$. Portanto, $\mathrm{C}_{\ell_{\max }}$ é o maior autovalor da matriz $\mathbf{B}=-\boldsymbol{\Delta}^{T}(\ddot{\mathbf{L}})^{-1} \boldsymbol{\Delta}$ e $\boldsymbol{\ell}_{\max }$ denota o autovetor correspondente.

O gráfico de $\boldsymbol{\ell}_{\max }$ contra a ordem das observações pode revelar quanto deve ser perturbado no modelo de modo a se obter uma variação desproporcional na estimativa $\hat{\boldsymbol{\beta}}$. Em particular se o interesse é apenas com relação ao subvetor $\boldsymbol{\beta}_{1}$, a curvatura normal na direção $\boldsymbol{\ell}$ fica dada por $\mathrm{C}_{\boldsymbol{\ell}}\left(\boldsymbol{\beta}_{1}\right)=2\left|\boldsymbol{\ell}^{T} \boldsymbol{\Delta}^{T}\left(\ddot{\mathbf{L}}^{-1}-\mathbf{B}_{1}\right) \boldsymbol{\Delta} \boldsymbol{\ell}\right|$, sendo

$$
\mathbf{B}_{1}=\left[\begin{array}{ll}
0 & 0 \\
0 & \ddot{\mathbf{L}}_{22}
\end{array}\right]
$$

com $-\ddot{\mathbf{L}}_{22}=\mathbf{X}^{T} \mathbf{V}_{2} \mathbf{X}$ sendo a matriz de informação de Fisher para $\boldsymbol{\beta}_{2}$. O gráfico do maior autovetor de $-\boldsymbol{\Delta}^{T}\left(\ddot{\mathbf{L}}^{-1}-\mathbf{B}_{1}\right) \boldsymbol{\Delta}$ contra a ordem das observações pode revelar os pontos com maior influência em $\hat{\boldsymbol{\beta}}_{1}$. De forma similar, se o interesse é apenas com respeito ao sub-vetor $\boldsymbol{\beta}_{2}$, a curvatura na direção $\boldsymbol{\ell}$ fica dada por $\mathrm{C}_{\ell}\left(\boldsymbol{\beta}_{2}\right)=2\left|\ell^{T} \boldsymbol{\Delta}^{T}\left(\ddot{\mathbf{L}}^{-1}-\mathbf{B}_{2}\right) \boldsymbol{\Delta} \ell\right|$, em que

$$
\mathbf{B}_{2}=\left[\begin{array}{ll}
\ddot{\mathbf{L}}_{11} & 0 \\
0 & 0
\end{array}\right]
$$

com $-\ddot{\mathbf{L}}_{11}=\mathbf{X}^{T} \mathbf{V}_{1} \mathbf{X}$ sendo a matriz de informação de Fisher para $\boldsymbol{\beta}_{1}$. A influência local das observações em $\boldsymbol{\beta}_{2}$ pode ser avaliada pelo gráfico de $\boldsymbol{\ell}_{\max }$ correspondente ao maior autovalor de $-\boldsymbol{\Delta}^{T}\left(\ddot{\mathbf{L}}^{-1}-\mathbf{B}_{1}\right) \boldsymbol{\Delta}$ contra a ordem das observações. 
Consideraremos aqui o logaritmo da função de verossimilhança perturbada como sendo

$$
\mathrm{L}(\boldsymbol{\beta} \mid \boldsymbol{\omega})=\sum_{i=1}^{n} \omega_{i} \mathrm{~L}\left(z_{i} ; \pi_{i}\right)
$$

em que $0 \leq \omega_{i} \leq 1$ e $\mathrm{L}\left(z_{i} ; \pi_{i}\right)$ denota o logaritmo da função de verossimilhança correspondente à $i$-ésima observação. Note que $\omega_{i}=0$ significa que o $i$-ésimo ponto foi eliminado e $\boldsymbol{\omega}_{0}=(1,1, \ldots, 1)^{T}$. Mostra-se facilmente para esse tipo de perturbação que $\boldsymbol{\Delta}$ é uma matriz $2 p \times n$ dada por

$$
\boldsymbol{\Delta}=\left[\begin{array}{c}
\mathbf{X}^{T} \operatorname{diag}\left(\mathbf{z}_{1}-\hat{\boldsymbol{\pi}}_{1}\right) \\
\mathbf{X}^{T} \operatorname{diag}\left(\mathbf{z}_{2}-\hat{\boldsymbol{\pi}}_{2}\right)
\end{array}\right]
$$

Logo, deveremos diagonalizar a matriz $\mathbf{B}=\boldsymbol{\Delta}^{T} \mathbf{K}^{-1}(\hat{\boldsymbol{\beta}}) \boldsymbol{\Delta}$, em que $\mathbf{K}(\boldsymbol{\beta})$ é dada em (1.8). Portanto, teremos que encontrar o maior autovalor da matriz $\mathbf{B}$ e o correspondente autovetor unitário que será $\boldsymbol{\ell}_{\max }$. O gráfico de $\boldsymbol{\ell}_{\max }$ contra a ordem das observações poderá revelar aqueles pontos com maior influência local em $\operatorname{LD}(\boldsymbol{\omega})$ e consequentemente em $\hat{\boldsymbol{\beta}}$.

Similarmente às seções anteriores poderemos fazer uma analogia com o modelo logístico binomial considerando as expressões das curvaturas desse modelo para cada um dos submodelos que compõe o modelo logístico trinomial. Essas curvaturas serão dadas por

$$
\mathrm{C}_{\ell}^{(1)}\left(\boldsymbol{\beta}_{1}\right)=2\left|\boldsymbol{\ell}^{T} \boldsymbol{\Delta}_{1}^{T}\left(\ddot{\mathbf{L}}_{11}\right)^{-1} \boldsymbol{\Delta}_{1} \boldsymbol{\ell}\right| \quad \text { e } \quad \mathrm{C}_{\ell}^{(2)}\left(\boldsymbol{\beta}_{2}\right)=2\left|\boldsymbol{\ell}^{T} \boldsymbol{\Delta}_{2}^{T}\left(\ddot{\mathbf{L}}_{22}\right)^{-1} \boldsymbol{\Delta}_{2} \boldsymbol{\ell}\right|
$$

em que

$$
\boldsymbol{\Delta}_{1}^{T}=\left.\frac{\partial^{2} \mathrm{~L}(\boldsymbol{\beta})}{\partial \boldsymbol{\beta}_{1} \partial \boldsymbol{\omega}^{T}}\right|_{\hat{\boldsymbol{\beta}}, \omega_{0}}=\mathbf{X}^{T} \operatorname{diag}\left(\mathbf{z}_{1}-\hat{\boldsymbol{\pi}}_{1}\right) \quad \text { e } \quad \boldsymbol{\Delta}_{2}^{T}=\left.\frac{\partial^{2} \mathrm{~L}(\boldsymbol{\beta})}{\partial \boldsymbol{\beta}_{2} \partial \boldsymbol{\omega}^{T}}\right|_{\hat{\beta}, \omega_{0}}=\mathbf{X}^{T} \operatorname{diag}\left(\mathbf{z}_{2}-\hat{\boldsymbol{\pi}}_{2}\right) .
$$

Assim, as matrizes a serem diagonalizadas ficam dadas por

$$
\begin{aligned}
\mathbf{B}^{(1)} & =-\boldsymbol{\Delta}_{1}^{T} \ddot{\mathbf{L}}_{11}^{-1} \boldsymbol{\Delta}_{1}=\operatorname{diag}\left(\mathbf{z}_{1}-\hat{\boldsymbol{\pi}}_{1}\right)^{T} \mathbf{X}\left(\mathbf{X}^{T} \hat{\mathbf{V}}_{1} \mathbf{X}\right)^{-1} \mathbf{X}^{T} \operatorname{diag}\left(\mathbf{z}_{1}-\hat{\boldsymbol{\pi}}_{1}\right) \\
& =\operatorname{diag}\left\{\mathbf{r}_{1}\right\} \hat{\mathbf{H}}^{(1)} \operatorname{diag}\left\{\mathbf{r}_{1}\right\},
\end{aligned}
$$

em que $\mathbf{r}_{1}=\left(r_{11}, \ldots, r_{1 n}\right)^{T} \operatorname{com} r_{1 i}=\left(z_{1 i}-\hat{\pi}_{1 i}\right) / \sqrt{\hat{\pi}_{1 i}\left(1-\hat{\pi}_{1 i}\right)}$ 


$$
\begin{aligned}
\mathbf{B}^{(2)} & =-\boldsymbol{\Delta}_{2}^{T} \ddot{\mathbf{L}}_{22}^{-1} \boldsymbol{\Delta}_{2}=\operatorname{diag}\left(\mathbf{z}_{2}-\hat{\boldsymbol{\pi}}_{2}\right)^{T} \mathbf{X}\left(\mathbf{X}^{T} \hat{\mathbf{V}}_{2} \mathbf{X}\right)^{-1} \mathbf{X}^{T} \operatorname{diag}\left(\mathbf{z}_{2}-\hat{\boldsymbol{\pi}}_{2}\right) \\
& =\operatorname{diag}\left\{\mathbf{r}_{2}\right\} \hat{\mathbf{H}}^{(2)} \operatorname{diag}\left\{\mathbf{r}_{2}\right\},
\end{aligned}
$$

em que $\mathbf{r}_{2}=\left(r_{21}, \ldots, r_{2 n}\right)^{T} \operatorname{com} r_{2 i}=\left(z_{2 i}-\hat{\pi}_{2 i}\right) / \sqrt{\hat{\pi}_{2 i}\left(1-\hat{\pi}_{2 i}\right)}$.

O autovetor correspondente ao maior autovalor de $\mathbf{B}^{(1)}$ será denotado por $\boldsymbol{\ell}_{\max }^{(1)}$ e $\boldsymbol{\ell}_{\max }^{(2)}$ será o autovetor correspondente ao autovalor de maior valor de $\mathbf{B}^{(2)}$. Deve-se proceder os gráficos de $\boldsymbol{\ell}_{\max }^{(1)}$ e $\boldsymbol{\ell}_{\max }^{(2)}$ contra os valores ajustados $\hat{\pi}_{1 i}$ e $\hat{\pi}_{21}$, respectivamente.

\subsection{Influência Local Total}

Lesaffre e Verbeke (1998) segerem ao invés de considerar a direção de maior curvatura, as curvaturas nas direções de cada uma das observações. A curvatura na direção da $i$-ésima observação será dada por

$$
\mathrm{C}_{i}=2\left|\boldsymbol{\ell}_{i}^{T} \boldsymbol{\Delta}^{T}(\ddot{\mathbf{L}})^{-1} \boldsymbol{\Delta} \boldsymbol{\ell}_{i}\right|
$$

em que $\boldsymbol{\ell}_{i}$ é um vetor de zeros com 1 na $i$-ésima posição. Mostra-se facilmente para o modelo logístico trinomial que $\mathrm{C}_{i}=2\left|B_{i i}\right|$, em que $B_{i i}$ é o $i$-ésimo elemento da diagonal principal da matriz B. Note que $\mathbf{B}$ matriz $n \times n$. É sugerido o gráfico de $\mathrm{C}_{i}$ contra a ordem das observações. Deve-se considerar aqueles pontos tais que $\mathrm{C}_{i}>2 \overline{\mathrm{C}}$, em que $\overline{\mathrm{C}}$ é o valor médio dos $\mathrm{C}_{i}$ 's.

Por outro lado, podemos considerar as expressões das curvaturas dos sub-modelos logísticos que compõem o modelo logístico trinomial. Essas curvaturas serão dadas por

$$
\mathrm{C}_{i}^{(1)}=2\left|\boldsymbol{\ell}_{i}^{T} \boldsymbol{\Delta}_{1}^{T}\left(\ddot{\mathbf{L}}_{11}\right)^{-1} \boldsymbol{\Delta}_{1} \boldsymbol{\ell}_{i}\right| \quad \text { e } \quad \mathrm{C}_{i}^{(2)}=2\left|\boldsymbol{\ell}_{i}^{T} \boldsymbol{\Delta}_{2}^{T}\left(\ddot{\mathbf{L}}_{22}\right)^{-1} \boldsymbol{\Delta}_{2} \boldsymbol{\ell}_{i}\right|
$$

Verifica-se facilmente que $\mathrm{C}_{i}^{(1)}=2\left|\mathrm{~B}_{i i}^{(1)}\right|$ e $\mathrm{C}_{i}^{(2)}=2\left|\mathrm{~B}_{i i}^{(2)}\right|$, em que $\mathrm{B}_{i i}^{(1)}$ e $\mathrm{B}_{i i}^{(2)}$ denotam, respectivamente, o $i$-ésimo elemento da diagonal principal de $\mathbf{B}^{(1)}$ e $\mathbf{B}^{(2)}$. A sugestão é construir os gráficos de $\mathrm{C}_{i}^{(1)}$ contra $\hat{\pi}_{1 i}$ e $\mathrm{C}_{i}^{(2)}$ contra $\hat{\pi}_{2 i}$ para avaliar observações influentes em $\hat{\boldsymbol{\beta}}_{1}$ e $\hat{\boldsymbol{\beta}}_{2}$, respectivamente. A sugestão é olhar com atenção aqueles pontos tais que $\mathrm{C}_{i}^{(1)}>2 \overline{\mathrm{C}}^{(1)}$ e $\mathrm{C}_{i}^{(2)}>2 \overline{\mathrm{C}}^{(2)}$. 
Desenvolvendo as expressões para $\mathrm{C}_{i}^{(1)}$ e $\mathrm{C}_{i}^{(2)}$ mostra-se facilmente que

$$
\mathrm{C}_{i}^{(1)}=2 \mathrm{r}_{1 i}^{2} \hat{h}_{i i}^{(1)} \quad \text { e } \quad \mathrm{C}_{i}^{(2)}=2 \mathrm{r}_{2 i}^{2} \hat{h}_{i i}^{(2)}
$$

\subsection{Resíduos}

Tradicionalmente a definição mais intuitiva de resíduo é dada pela diferença $\left(y_{i}-\hat{y}_{i}\right)$, que mede de forma linear quão o valor predito se afasta do valor observado. Essa definição funciona bem no caso linear, embora seja necessário sempre obter a forma padronizada da diferença, uma vez que as variâncias não são as mesmas. Além disso, mesmo que os erros sejam independentes os resíduos são em geral correlacionados, exigindo algum cuidado na hora de interpretá-los. Nesse sentido, Atkinson (1981, 1985) propõe, quando os resíduos são confrontados com alguma distribuição postulada o uso de envelopes, que são bandas de confiança obtidas pelo método de Monte Carlo a partir do modelo ajustado para avaliarem afastamentos sérios da distribuição postulada para os resíduos e de forma indireta da distribuição postulada para os erros.

Os resíduos, contudo, mais utilizados em modelos lineares generalizados são definidos a partir dos componentes da função desvio, que pode ser interpretada como foi visto na Seção 1.4 como sendo uma distância entre o logaritmo da verossimilhança do modelo saturado e o logaritmo da verossimilhança do modelo postulado. A versão padronizada (vide, por exemplo, McCullagh, 1987) é a seguinte:

$$
t_{d_{i}}=\frac{ \pm d\left(y_{i} ; \hat{\mu}_{i}\right)}{\left(1-\hat{h}_{i i}\right)^{1 / 2}}
$$

em que $d^{2}\left(y_{i} ; \hat{\mu}_{i}\right)$ é o i-ésimo componente do desvio, $\hat{h}_{i i}$ é o elemento da diagonal principal da matriz de projeção correspondente e o sinal atribuído é o mesmo de $\left(y_{i}-\hat{y}_{i}\right)$. Williams (1984) verificou através de estudos de simulação que a distribuição empírica está próxima da normalidade para vários MLGs. McCullagh (1987, p. 214) mostra para os MLGs que 
a distribuição de probabilidade de

$$
\frac{d\left(Y_{i} ; \eta_{i}\right)+\rho_{3 i} / 6}{\sqrt{1+\left(14 \rho_{3 i}^{2}-9 \rho_{4 i}\right) / 36}}
$$

é aproximadamente $N(0,1)$, em que $\rho_{3 i}$ e $\rho_{4 i}$ são os coeficientes de assimetria e curtose de $\partial L\left(\eta_{i}\right) / \partial \eta_{i}$, respectivamente, e $\eta_{i}$ é o preditor linear. É possível mostrar para os MLGs usando resultados de Cox e Snell (1968) que $\mathrm{E}\left\{\mathrm{d}\left(\mathrm{Y}_{\mathrm{i}} ; \eta_{\mathrm{i}}\right)\right\}=0$ e $\operatorname{Var}\left\{\mathrm{d}\left(\mathrm{Y}_{\mathrm{i}} ; \eta_{\mathrm{i}}\right)\right\}=1-\mathrm{h}_{\mathrm{ii}}$, em que os termos negligenciados são de $O\left(n^{-1}\right)$. Esses resultados reforçam o uso da padronização acima para $t_{d_{i}}$.

No caso do modelo logístico trinomial o componente do desvio assume a forma:

- $d\left(y_{i} ; \hat{\mu}_{i}\right)=\sqrt{2}\left\{a_{0 i} \log \left(a_{0 i} / n_{i} \hat{\pi}_{0 i}\right)+a_{1 i} \log \left(a_{1 i} / n_{i} \hat{\pi}_{1 i}\right)+a_{2 i} \log \left(a_{2 i} / n_{i} \hat{\pi}_{2 i}\right)\right\}$ se $0<a_{0 i}, a_{1 i}, a_{2 i}<n_{i}$,

- $d\left(y_{i} ; \hat{\mu}_{i}\right)=\sqrt{-2 n_{i} \log \left(\hat{\pi}_{0 i}\right)} \quad$ se $\quad\left(a_{0 i}=n_{i}, a_{1 i}=a_{2 i}=0\right)$,

- $d\left(y_{i} ; \hat{\mu}_{i}\right)=\sqrt{-2 n_{i} \log \left(\hat{\pi}_{1 i}\right)} \quad$ se $\quad\left(a_{1 i}=n_{i}, a_{0 i}=a_{2 i}=0\right) \mathrm{e}$

- $d\left(y_{i} ; \hat{\mu}_{i}\right)=\sqrt{-2 n_{i} \log \left(\hat{\pi}_{2 i}\right)} \quad$ se $\quad\left(a_{2 i}=n_{i}, a_{0 i}=a_{1 i}=0\right)$.

Como fica difícil a atribuição de um sinal para $d\left(y_{i} ; \hat{\mu}_{i}\right)$ uma vez que temos três diferenças para o $i$-ésimo elemento, $\left(a_{0 i}-n_{i} \hat{\pi}_{0 i}\right),\left(a_{1 i}-n_{i} \hat{\pi}_{1 i}\right)$ e $\left(a_{2 i}-n_{i} \hat{\pi}_{2 i}\right)$ deixaremos o resíduo componente do desvio sem sinal.

O principal desafio técnico neste caso é encontrar o fator de correção para a padronização do resíduo componente do desvio. Como isso envolve cálculos complicados abrangendo expansões de Cox e Snell (1968) podemos propor algum tipo de correção empírica envolvendo as quantidades $\hat{h}_{i i}^{(1)}, \hat{h}_{i i}^{(2)}, \hat{m}_{i i}^{(1)}$ e $\hat{m}_{i i}^{(2)}$ propostas ne seção 2.2. Uma sugestão mais simples seria considerar como fator de correção a quantidade $\sqrt{1-\bar{h}_{i i}}$, em que $\bar{h}_{i i}=\left(\hat{h}_{i i}^{(1)}+\hat{h}_{i i}^{(2)}\right) / 2$. 
Assim, o resíduo componente do desvio padronizado ficaria dado por

$$
t_{d_{i}}=\frac{d\left(y_{i} ; \hat{\mu}_{i}\right)}{\sqrt{1-\bar{h}_{i i}}}
$$

Resta saber empiricamente qual seria a distribuição de $t_{d_{i}}$. Contudo, o uso de envelopes conforme sugerido por Atkinson (1981) (vide também Williams, 1987) pode contornar o problema de não se saber a distribuição aproximada de $t_{d_{i}}$. O envelope consiste basicamente numa banda de confiança empírica para $t_{d_{i}}$ construída através do modelo ajustado que pode ajudar na detecção de observações aberrantes segundo $t_{d_{i}}$, bem como afastamentos sérios da distribuição postulada para a resposta ou mesmo superdispersão, variâncias maiores do que aquelas postuladas para o modelo.

Um outro resíduo que poderia ser usado no caso trinomial é o resíduo de Pearson padronizado definido na seção 2.3. Nesse caso seriam calculados dois grupos de resíduos, um para cada sub-modelo logístico. Assim, seriam calculados os resíduos referentes às categorias $Y=1$ e $Y=2$, os quais são, respectivamente, definidos por

$$
t_{S_{i}}^{(1)}=\frac{\left(a_{1 i}-n_{1 i} \hat{\pi}_{1 i}\right)}{\sqrt{n_{1 i} \hat{\pi}_{1 i}\left(1-\hat{\pi}_{1 i}\right)\left(1-\hat{h}_{i i}^{(1)}\right)}} \quad \text { e } \quad t_{S_{i}}^{(2)}=\frac{\left(a_{2 i}-n_{2 i} \hat{\pi}_{2 i}\right)}{\sqrt{n_{2 i} \hat{\pi}_{2 i}\left(1-\hat{\pi}_{2 i}\right)\left(1-\hat{h}_{i i}^{(2)}\right)}} .
$$

Como é mais razoável esperar que $t_{S_{i}}^{(1)}$ e $t_{S_{i}}^{(2)}$ sejam aproximadamente normais de média zero e variância um quando $n_{1 i}$ e $n_{2 i}$ não são pequenos, algumas técnicas usuais de diagnóstico podem ser usadas com mais segurança. Por exemplo, os gráficos de $t_{S_{i}}^{(1)}$ e $t_{S_{i}}^{(2)}$ contra os valores ajustados $\hat{\pi}_{1 i}$ e $\hat{\pi}_{2 i}$, respectivamente, podem revelar quais observações são marginalmente aberrantes em cada sub-modelo. Nesse caso também poderiam ser atribuídos envelopes para cada um dos resíduos $t_{S_{i}}^{(1)}$ e $t_{S_{i}}^{(2)}$. Esses envelopes embora levem em conta resíduos de sub-modelos logísticos devem ser gerados do modelo logístico trinomial ajustado. 


\section{Capítulo 3}

\section{Aplicações}

\subsection{Introdução}

Neste capítulo vamos aplicar as diversas técnicas de diagnóstico desenvolvidas no capítulo 2 aos exemplos vistos no capítulo 1. Iremos ainda exibir um painel comparativo onde serão confrontadas as técnicas que serão aplicadas aos sub-modelos do modelo trinomial com os dignósticos desenvolvidos diretamente para este modelo.

Como acontece com qualquer modelo ajustado, antes de utilizá-lo e fazer inferências devemos avaliar seu ajuste final e examinar sua contribuição para cada um dos indivíduos. Na regressão logística trinomial este passo é bem mais complicado do que no caso binomial. Quando nosso modelo tem respostas binárias temos apenas um valor ajustado, a probabilidade logística da resposta é dada por $\mathrm{P}(\mathrm{Y}=1 \mid \mathbf{x})$. Quando a variável resposta tem três categorias teremos duas probabilidades a serem estimadas, $P(Y=1 \mid \mathbf{x})$ e $P(Y=2$ $\mid \mathbf{x})$.

Para esta avaliação vamos utilizar a abordagem de Hosmer e Lemeshow que recomendam que avaliemos os dois sub-modelos calculando as medidas de diagnósticos usando as regressões logísticas individuais, a seguir os resultados devem ser integrados levando em consideração os efeitos dos pontos influentes em cada modelo. Em particular, os pontos que são influentes em apenas um dos sub-modelos devem ser examinados com mais 
atenção.

\subsection{Exemplo 1}

Este exemplo avalia fatores associados ao conhecimento, atitude e comportamento de mulheres em relação ao exame de mamografia, os ajustes finais são dados abaixo

$$
\begin{aligned}
\log \left\{\frac{\hat{\pi}_{1 i}}{\hat{\pi}_{0 i}}\right\}= & -2,7024+2,0950 \times \text { SintomaD }_{i}-0,2510 \times \text { Beneficio }_{i}+ \\
& 1,2933 \times \text { Historico }_{i}+1,2439 \times \text { Autoexame }_{i}+0,0893 \times \text { Detectar }_{2 i}+ \\
& 0,9719 \times \text { Detectar }_{3 i}
\end{aligned}
$$

$\mathrm{e}$

$$
\begin{aligned}
\log \left\{\frac{\hat{\pi}_{2 i}}{\hat{\pi}_{0 i}}\right\}= & -0,9987+1,1214 \times \text { SintomaD }_{i}-0,1681 \times \text { Beneficio }_{i}+ \\
& 1,0140 \times \text { Historico }_{i}+1,0286 \times \text { Autoexame }_{i}-0,9022 \times \text { Detectar }_{2 i}- \\
& 0,6698 \times \text { Detectar }_{3 i} .
\end{aligned}
$$

O primeiro sub-modelo compara mulheres que realizaram o exame de mamografia nos últimos 12 meses (Experiência=1) ao grupo de mulheres que nunca realizaram o exame (Experiência=0) enquanto que o segundo sub-modelo compara o grupo de mulheres que submeteram-se ao exame a mais de um ano (Experiência=2) ao grupo de mulheres que nunca realizaram o exame (Experiência=0).

As estatísticas de qualidade do ajuste de Hosmer-Lemeshow apresentaram níveis descritivos de 0,55 para o sub-modelo 1 e 0,11 para o sub-modelo 2 , ou seja, $C_{1}^{H L}$ e $C_{2}^{H L}$ apresentam valores não significativos, indicando que ambos os modelos estão satisfatoriamente ajustados.

Ainda sob a ótica de Hosmer e Lemeshow vamos construir os gráficos de diagnóstico para os dois sub-modelos estimados. 
Apresentaremos alguns gráficos de diagnóstico. Inicialmente os gráfico de $\hat{h}_{i i}^{(1)}$ e $\hat{h}_{i i}^{(2)}$ contra as probabilidades ajustadas $\hat{\pi}_{1 i}$ e $\hat{\pi}_{2 i}$, respectivamente (Figuras 3.1 e 3.2). Como podemos notar nesses dois gráficos três mulheres se destacam com um valor relativamente alto para as medidas de alavanca, \#83 e \#12 (Figura 3.1) e \#241 (Figura 3.2). Em seguida são apresentadas as medidas aproximadas dos afastamentos da verossimilhança $\mathrm{LD}_{i}^{(1)}$ e $\mathrm{LD}_{i}^{(2)}$ contra as probabilidades ajustadas $\hat{\pi}_{1 i}$ e $\hat{\pi}_{2 i}$, respectivamente (Figuras 3.3 e 3.4). Destacam-se na primeira figura as mulheres \#80 e \#293 e na segunda figura as mulheres \#90 e \#260. Essas quatro mulheres têm em comum o fato de terem probabilidades ajustadas baixas (menores que 0,20 ) para $\pi_{1}$ e $\pi_{2}$, porém tiveram respostas observadas $\mathrm{Y}=1$ ou $\mathrm{Y}=2$. Depois são apresentados nas Figuras 3.5 e 3.6 os gráficos de resíduos de Pearson padronizados $t_{S_{i}}^{(1)}$ e $t_{S_{i}}^{(2)}$ contra as probabilidades ajustadas $\hat{\pi}_{1 i}$ e $\hat{\pi}_{2 i}$, respectivamente. Podemos notar por essas figuras e principalmente pelos histogramas apresentados nas Figuras 3.7 e 3.8 uma acentuada assimetria na distribuição empírica desses resíduos, dificultando a definição de critérios para classificação de pontos aberrantes. Esse tipo de comportamento já era esperado nesse exemplo uma vez que não se tem réplicas, ou seja, $n_{i}=1, \forall_{i}$. A distribuição de $t_{S_{i}}$ se aproxima de uma normal padrão quando $n_{i}$ não é pequeno, $\forall_{i}$. Partimos então para o desenvolvimento do gráfico do resíduo do componente do desvio $t_{d_{i}}$ (Figura 3.11) contra a ordem das observações. Como não foi atribuído sinal para esse resíduo, olhamos apenas aqueles pontos que se destacaram com os maiores valores que correspondem às mulheres \#80 e \#293 que já haviam sido destacados em figuras anteriores. 
Tabela 3.1

Pontos por sub-modelo para cada medida de diagnóstico.

\begin{tabular}{ccll}
\hline \hline Gráfico & Sub-modelo & Medida de diagnóstico & Pontos a serem observados \\
\hline 3.1 & 1 & Alavanca - binomial & $\# 12$ e \#83 \\
3.3 & 1 & Alavanca - trinomial & $\# 12$ e \#83 \\
3.5 & 1 & Afastamento da verossimilhança & $\# 80$ e \#293 \\
3.11 & 1 & Influência local total - binomial & $\# 83$ e \#234 \\
3.2 & 2 & Alavanca - binomial & $\# 241$ \\
3.4 & 2 & Alavanca - trinomial & $\# 241, \# 279$ e \#382 \\
3.6 & 2 & Afastamento da verossimilhança & $\# 90$ e \#260 \\
3.8 & 2 & Influência local - binomial & $\# 128$ e \#260 \\
3.12 & 2 & Influência local total - binomial & $\# 260$ \\
3.9 & ambos & Influência local - trinomial & $\# 39$ e \#108 \\
3.10 & ambos & Influência local total - trinomial & $\# 80$ e \#293 \\
3.13 & ambos & Componente do desvio - trinomial & $\# 80$ e \#293 \\
\hline \hline
\end{tabular}

A tabela acima mostra os pontos que foram detectados como possíveis observações influentes e/ou aberrantes, em cada um dos gráficos para cada sub-modelo avaliado.

Para auxiliar na análise vamos traçar um perfil de cada uma das mulheres:

Observação \#12 - Nunca realizou o exame, acha necessário o exame mesmo antes de desenvolver os sintomas, tem histórico de câncer familiar, não sabe como se auto-examinar, acha muito provável que o exame venha a detectar a doença e concorda fortemente com o benefício do exame. Probabilidades ajustadas $\hat{\pi}_{1}=0,424$ e $\hat{\pi}_{2}=0,212$.

Observação \#83 - Submeteu-se ao exame nos últimos 12 meses, acha necessário o exame mesmo antes de desenvolver os sintomas, tem histórico de câncer familiar, não sabe como se auto-examinar, acha muito provável que o exame venha a detectar a doença e concorda fortemente com o benefício do exame. Probabilidades ajustadas $\hat{\pi}_{1}=0,378$ e $\hat{\pi}_{2}=0,205$.

Observação \#80 - Submeteu-se ao exame nos últimos 12 meses, não acha necessário o exame antes de desenvolver os sintomas, não tem histórico de câncer familiar, não sabe como se auto-examinar, acha provável que o exame venha a detectar a doença e concorda 
fortemente com o benefício do exame. Probabilidades ajustadas $\hat{\pi}_{1}=0,015$ e $\hat{\pi}_{2}=0,051$.

Observação \#293 - Submeteu-se ao exame nos últimos 12 meses, não acha necessário o exame antes de desenvolver os sintomas, não tem histórico de câncer familiar, sabe como se auto-examinar, acha pouco provável que o exame venha a detectar a doença e concorda parcialmente com o benefício do exame. Probabilidades ajustadas $\hat{\pi}_{1}=0,016$ e $\hat{\pi}_{2}=0,158$.

Observação \#234 - Submeteu-se ao exame nos últimos 12 meses, acha necessário o exame mesmo antes de desenvolver os sintomas, não tem histórico de câncer familiar, não sabe como se auto-examinar, acha muito provável que o exame venha a detectar a doença e concorda fortemente com o benefício do exame. Probabilidades ajustadas $\hat{\pi}_{1}=0,247$ e $\hat{\pi}_{2}=0,150$.

Observação \#241 - Nunca realizou o exame, acha necessário o exame mesmo antes de desenvolver os sintomas, tem histórico de câncer familiar, não sabe como se auto-examinar, acha pouco provável que o exame venha a detectar a doença e concorda parcialmente com o benefício do exame. Probabilidades ajustadas $\hat{\pi}_{1}=0,078$ e $\hat{\pi}_{2}=0,303$.

Observação \#382 - Submeteu-se ao exame a mais de um ano, acha necessário o exame mesmo antes de desenvolver os sintomas, não tem histórico de câncer familiar, sabe como se auto-examinar, acha muito provável que o exame venha a detectar a doença e concorda com o benefício do exame. Probabilidades ajustadas $\hat{\pi}_{1}=0,278$ e $\hat{\pi}_{2}=0,190$.

Observação \#279 - Nunca realizou o exame, não acha necessário o exame antes de desenvolver os sintomas, tem histórico de câncer familiar, sabe como se auto-examinar, acha pouco provável que o exame venha a detectar a doença e concorda parcialmente com o benefício do exame. Probabilidades ajustadas $\hat{\pi}_{1}=0,036$ e $\hat{\pi}_{2}=0,298$.

Observação \#90 - Submeteu-se ao exame a mais de um ano, não acha necessário o exame antes de desenvolver os sintomas, não tem histórico de câncer familiar, não sabe como se auto-examinar, acha pouco provável que o exame venha a detectar a doença e concorda parcialmente com o benefício do exame. Probabilidades ajustadas $\hat{\pi}_{1}=0,006$ e $\hat{\pi}_{2}=0,075$. 
Observação \#260 - Submeteu-se ao exame a mais de um ano, acha necessário o exame antes de desenvolver os sintomas, não tem histórico de câncer familiar, não sabe como se auto-examinar, acha muito provável que o exame venha a detectar a doença e concorda com o benefício do exame. Probabilidades ajustadas $\hat{\pi}_{1}=0,035$ e $\hat{\pi}_{2}=0,168$.

Observação \#128 - Submeteu-se ao exame a mais de um ano, acha necessário o exame antes de desenvolver os sintomas, não tem histórico de câncer familiar, sabe como se autoexaminar, acha pouco provável que o exame venha a detectar a doença e concorda com o benefício do exame. Probabilidades ajustadas $\hat{\pi}_{1}=0,074$ e $\hat{\pi}_{2}=0,308$.

Observação \#39 - Nunca realizou o exame, acha necessário o exame mesmo antes de desenvolver os sintomas, tem histórico de câncer familiar, sabe como se auto-examinar, acha muito provável que o exame venha a detectar a doença e concorda fortemente com o benefício do exame. Probabilidades ajustadas $\hat{\pi}_{1}=0,639$ e $\hat{\pi}_{2}=0,237$.

Observação \#108 - Nunca realizou o exame, acha necessário o exame mesmo antes de desenvolver os sintomas, tem histórico de câncer familiar, sabe como se auto-examinar, acha muito provável que o exame venha a detectar a doença e concorda fortemente com o benefício do exame. Probabilidades ajustadas $\hat{\pi}_{1}=0,639$ e $\hat{\pi}_{2}=0,237$.

Os pontos que se destacaram nos gráficos de alavanca serão considerados possivelmente influentes se os valores de $\hat{h}_{i i}$ destoarem de seus valores esperados, conforme tabela abaixo:

Tabela 3.2

Pontos de alavanca por sub-modelo.

\begin{tabular}{cccc}
\hline \hline Sub-modelo & Observação & Valor de $\hat{\pi}_{i}$ & Valor esperado de $\hat{h}_{i i}$ \\
\hline 1 & $\# 12$ & 0,4239 & Pequeno para $\pi_{i}$ entre 0,3 e 0,7 \\
1 & $\# 83$ & 0,3777 & Pequeno para $\pi_{i}$ entre 0,3 e 0,7 \\
2 & $\# 241$ & 0,3035 & Pequeno para $\pi_{i}$ entre 0,3 e 0,7 \\
\hline \hline
\end{tabular}

Para os três pontos (\#12, \#83 e \#241) que se destacaram o valor de $\hat{h}_{i i}$ esperado deve ser pequeno segundo o critério de Hosmer e Lemeshow (1989), porém os valores 
encontrados podem ser considerados altos uma vez que $\bar{h} \cong 2 p / n=0,034$. Assim, essas três mulheres podem ter alguma influência nos resultados dos modelos. Além desses pontos o gráfico de alavanca detectou tambám no sub-modelo 2 as observações \#279 e \#382.

As observações \#80 e \#293 da Figura 3.5 (sub-modelo 1) e \#90 e \#260 da Figura 3.6 (sub-modelo 2) parecem destoar das demais segundo $\mathrm{LD}_{i}$. Se concluirmos que a variação que essas observações causam nas estimativas são muito desproporcionais, podemos afirmar que esses pontos são influentes. Porém, deve-se verificar ainda se a eliminação dos mesmos leva a mudanças inferenciais, deixando, por exemplo, algum coeficiente não significativo. Note ainda que esses mesmos pontos foram detectados pelo gráfico de Influência local total trinomial Figura 3.10 e pelo gráfico de resíduos de componente do desvio trinomila Figura 3.13.

Iremos apesentar a seguir três tabelas em que retiramos as observações destacadas acima individualmente e verificaremos as variações percentuais nos coeficientes estimados bem como os novos níveis descritivos. Assim, poderemos verificar se as variações ocorridas nas estimativas levam a mudanças inferenciais, ou seja, se algum coeficiente deixa de ser significativo ou passa a ser significativo. Na Tabela 3.3 apresentamos esse estudo para as observações \#12, \#83 e \#241 que se destacaram nos gráficos de pontos de alavanca binomial. Nas Tabelas 3.4 e 3.5 são desenvolvidos estudos similares para as observações (\#80 e \#293) que se destacaram nos gráficos de $\mathrm{LD}_{i}^{(1)}, \mathrm{t}_{d_{i}}$ e $\mathrm{C}$ e ainda para as observações (\#90 e \#260) que se destacaram nos gráficos de $\mathrm{LD}_{i}^{(2)}, \mathrm{C}_{l}^{(2)}$ e $\mathrm{C}_{i}^{(2)}$ para os sub-modelos 1 e 2 , respectivamente.

Antes de avaliarmos as variações das estimativas e níveis descritivos que serão exibidos pelas tabelas a seguir, vale lembrar (como foi visto no capítulo 1) que a variável explicativa Detectar foi avaliada como não-significativa pela estatística de Wald (vide Tabela 1.6). Apesar disso ela permaneceu no modelo devido a sua influência confundidora na variável SintomaD. 
Inicialmente iremos considerar a retirada das observações \#12, \#83 e \#241 (Tabela 3.3). Além das fortes variações nas estimativas observadas nos coeficientes da váriavel explicativa Detectar, notamos também variações nas estimativas dos coeficientes das variáveis Hitorico e Autoexame. Esse comportamente entretanto não implica em mudança inferencial para a variável Hitorico nem na variável Detectar. Já para a variável Autoexame notamos mudança na significância de seu coeficiente, variando o nível descritivo de $\simeq 4 \%$ para $\simeq 10 \%$ no sub-modelo 2 . Isso significa dizer que a retirada das observações \#12, \#83 e \#241 afeta o sub-modelo 2 tornando a variável Autoexame não significativa para o mesmo se considerarmos um nível de significância, por exemplo, de $5 \%$.

Consideramos agora a retirada das observações \#80 e \#293. A Tabela 3.4 exibe as variáveis SintomaD e Autoexame apresentando variações destacadas no sub-modelo 1 principalmente quando retiramos as duas observações (\#80 e \#293) simultaneamente dos dados. Note ainda que a retirada dessas observações pouco influencia nas estimativas para o sub-modelo 2, isso parece fazer sentido, uma vez, que esses pontos mostraram-se influentes apenas no sub-modelo 1. 
Tabela 3.3

Variação nas estimativas e níveis descritivos eliminando as observações \#12, \#83 e \#241.

\begin{tabular}{clrc}
\hline \hline \multirow{3}{*}{ Sub-modelo } & Var. & $\begin{array}{c}\text { Nível } \\
\text { descritivo }\end{array}$ \\
\hline 1 & SintomaD & 0 & 0,0001 \\
& Beneficio & -1 & 0,0005 \\
& Historico & 5 & 0,0032 \\
& Autoexame & 1 & 0,0276 \\
& Detectar $_{2}$ & -166 & 0,9597 \\
& Detectar $_{3}$ & 16 & 0,4707 \\
& Intercepto & -5 & 0,0787 \\
\hline 2 & SintomaD & 3 & 0,0013 \\
& Beneficio & 0 & 0,0236 \\
& Historico & 14 & 0,0145 \\
& Autoexame & -17 & 0,0970 \\
& Detectar $_{2}$ & -12 & 0,1603 \\
& Detectar $_{3}$ & -16 & 0,2652 \\
& Intercepto $^{2}$ & 23 & 0,4719 \\
\hline \hline
\end{tabular}

Apesar das variações desproporcionais observadas não notamos mudanças inferenciais significativas, levando-nos a não considerar a retirada desses pontos.

As Figuras 3.5, 3.10 e 3.13, apresentam as mesmas observações \#80 e \#293 em destaque, esse pontos foram estudados na Tabela 3.4.

Na Tabela 3.5 podemos notar que as variáveis SintomaD, Historico e Autoexame apresentam variações em seus coeficientes, sendo que essas variações ficam mais evidentes no sub-modelo 2. quando as duas observações (\#90, \#260) são retiradas simultaneamente dos dados. 
Tabela 3.4

Variação nas estimativas e níveis descritivos eliminando \#80, \#293 e ambas.

\begin{tabular}{clrcrrrc}
\hline \hline \multirow{3}{*}{ Sub-modelo } & Var. & $\begin{array}{c}\text { Nível } \\
\text { Variável }\end{array}$ & $\%$ & $\begin{array}{r}\text { Var. } \\
\text { descritivo }\end{array}$ & $\begin{array}{c}\text { Nível } \\
\text { descritivo }\end{array}$ & $\begin{array}{r}\text { Var. } \\
\%\end{array}$ & $\begin{array}{c}\text { Nível } \\
\text { descritivo }\end{array}$ \\
\hline \multirow{2}{*}{1} & SintomaD & 10 & 0,0001 & 10 & 0,0001 & 21 & 0,0001 \\
& Beneficio & -4 & 0,0011 & -2 & 0,0005 & 2 & 0,0010 \\
& Historico & 3 & 0,0024 & 5 & 0,0022 & 8 & 0,0019 \\
& Autoexame & 23 & 0,0075 & -3 & 0,0233 & 21 & 0,0095 \\
& Detectar $_{2}$ & -136 & 0,9779 & 11.965 & 0,9547 & 11.809 & 0,9546 \\
& Detectar $_{3}$ & -1 & 0,3942 & 1.098 & 0,9510 & 1.096 & 0,9504 \\
& Intercepto $^{2}$ & -19 & 0,0287 & -399 & 0,9433 & -420 & 0,9400 \\
\hline & SintomaD & 1 & 0,0015 & 0 & 0,0017 & 1 & 0,0015 \\
& Beneficio $^{2}$ & -1 & 0,0255 & 0 & 0,0231 & 1 & 0,0252 \\
& Historico & 2 & 0,0236 & 2 & 0,0232 & 4 & 0,0215 \\
& Autoexame & 3 & 0,0389 & -2 & 0,0486 & 2 & 0,0417 \\
& Detectar & -2 & 0,1979 & -14 & 0,2770 & -12 & 0,2691 \\
& Detectar $_{3}$ & 0 & 0,3319 & -19 & 0,4277 & -20 & 0,4348 \\
& Intercepto & -5 & 0,3279 & -10 & 0,3049 & -16 & 0,2813 \\
\hline \hline
\end{tabular}

Tabela 3.5

Variação nas estimativas e níveis descritivos eliminando \#90, \#260 e ambas.

\begin{tabular}{|c|c|c|c|c|c|c|c|}
\hline Sub-modelo & Variável & $\begin{array}{r}\text { Var. } \\
\%\end{array}$ & $\begin{array}{c}\text { Nível } \\
\text { descritivo }\end{array}$ & $\begin{array}{r}\text { Var. } \\
\%\end{array}$ & $\begin{array}{c}\text { Nível } \\
\text { descritivo }\end{array}$ & $\begin{array}{r}\text { Var. } \\
\%\end{array}$ & $\begin{array}{c}\text { Nível } \\
\text { descritivo }\end{array}$ \\
\hline \multirow[t]{7}{*}{1} & SintomaD & 1 & 0,0001 & 0 & 0,0001 & 0 & 0,0001 \\
\hline & Beneficio & 0 & 0,0006 & 0 & 0,0006 & 0 & 0,0006 \\
\hline & Historico & 2 & 0,0025 & 1 & 0,0026 & 4 & 0,0021 \\
\hline & Autoexame & 3 & 0,0153 & 2 & 0,0163 & 5 & 0,0132 \\
\hline & Detectar $_{2}$ & 167 & 0,8373 & 117 & 0,8676 & 309 & 0,7529 \\
\hline & Detectar $_{3}$ & 12 & 0,3193 & 11 & 0,3392 & 28 & 0,2676 \\
\hline & Intercepto & -7 & 0.0438 & -4 & 0,0505 & -12 & 0,0351 \\
\hline \multirow[t]{7}{*}{2} & SintomaD & 7 & 0,0010 & -3 & 0,0015 & 5 & 0,0015 \\
\hline & Beneficio & 1 & 0,0290 & -1 & 0,0226 & 1 & 0,0277 \\
\hline & Historico & 6 & 0,0194 & 4 & 0,0204 & 11 & 0,0150 \\
\hline & Autoexame & 20 & 0,0273 & 18 & 0,0290 & 46 & 0,0167 \\
\hline & Detectar $_{2}$ & 56 & 0,5110 & 60 & 0,4733 & 950 & 0,9866 \\
\hline & Detectar $_{3}$ & 42 & 0,7031 & 111 & 0,6721 & 130 & 0,8016 \\
\hline & Intercepto & -69 & 0,1420 & -48 & 0,1939 & -140 & 0,0580 \\
\hline
\end{tabular}


Novamente as variações desproporcionais devidas à retirada das observações não levam a mudanças inferenciais significativas, levando-nos a não considerar a retirada destas observações.

Tem-se nas Figuras 3.9 e 3.10 os gráficos de $m_{i i}^{(1)}$ e $m_{i i}^{(2)}$ contra as probabilidades estimadas pelos sub-modelos 1 e 2, respectivamente. Como podemos notar, são as mesmas observações (\#12, \#83 e \#241) destacadas pelas medidas de alavanca (Figuras 3.1 e 3.2) considerando os dois sub-modelos, as quais foram estudadas na Tabela 3.3.

Ao analisarmos as duas estratégias de detecção de pontos aberrantes e/ou influentes, verificamos que as observações em destaque são as mesmas, dando indicação de que é indiferente utilizar a abordagem de Hosmer e Lemeshow que calcula essas medidas para os dois sub-modelos isoladamente ou medidas desenvolvidas neste trabalho diretamente para o modelo logístico trinomial.

Se compararmos as Figuras 3.1 com 3.9 e ainda 3.2 com 3.10, temos a impressão de tratar-se das mesmas medidas. Entretanto, existe uma sutil diferença entre elas, porém isso não altera nossa indicação apontada pela Tabela 3.2.

A Figura 3.11 do resíduo componente do desvio $t_{d_{i}}$ contra a ordem das observações, mostrou-se eficaz na detecção de pontos, embora essas mesmas observações já tivessem sido evidenciadas pela Figura 3.3 que exibe os afastamentos da verossimilhança.

\section{$3.3 \quad$ Exemplo 2}

Este exemplo investiga a associação entre resultado num teste respiratório, hábito de fumar e idade. Os ajustes finais são dados abaixo

$$
\begin{aligned}
& \log \left\{\frac{\hat{\pi}_{1 i}}{\hat{\pi}_{0 i}}\right\}=-3,8508+0,5380 \times \text { idade }_{i}+0,7881 \times \text { status }_{2_{i}}+0,8319 \times \text { status }_{3_{i}} \\
& \log \left\{\frac{\hat{\pi}_{2 i}}{\hat{\pi}_{0 i}}\right\}=-6,5231+1,3786 \times \text { idade }_{i}+0,8221 \times \text { status }_{2_{i}}+1,3463 \times \text { status }_{3_{i}} .
\end{aligned}
$$


O primeiro sub-modelo compara o grupo de trabalhadores que tiveram resultado indefinido no teste respiratório (Resultado=1) ao grupo de trabalhadores que tiveram resultado normal no teste respiratório (Resultado=0) enquanto que o segundo sub-modelo compara o grupo de trabalhadores que tiveram resultado anormal no teste respiratório (Resultado=2) ao grupo de trabalhadores que tiveram resultado normal no teste respiratório (Resultado=0).

As estatísticas de qualidade do ajuste de Hosmer-Lemeshow apresentaram níveis descritivos de 0,011 para o sub-modelo 1 e 0,037 para o sub-modelo 2 , ou seja, $C_{1}^{H L}$ e $C_{2}^{H L}$ apresentam valores significativos para $\alpha=5 \%$, indicando que ambos os modelos não estão satisfatoriamente ajustados.

Uma alternativa seria incluir no modelo a interação idade $\times$ status e avaliar a significância de $C_{1}^{H L}$ e $C_{2}^{H L}$.

Como temos um exemplo de dados agrupados perde-se um pouco o sentido a aplicação de gráficos de diagnóstico para avaliação do impacto individual das observações nas estimativas. Aqui as observações são grupos não necessariamente do mesmo tamanho. Assim, iremos apenas desenvolver gráficos de $t_{S_{i}}^{(1)}$ e $t_{S_{i}}^{(2)}$ contra as probabilidades ajustadas $\hat{\pi}_{1_{i}}$ e $\hat{\pi}_{2_{i}}$, respectivamente, que são apresentados nas Figuras 3.12 e 3.13. Como podemos notar apesar do exemplo contar com 2.219 respondentes, temos apenas seis agrupamentos o que dificulta qualquer estratégia de eliminação de pontos, uma vez que ao eliminarmos um grupo estaremos eliminando um grande número de observações, o que deverá implicar em mudanças não apenas nos coeficientes mas também em suas significâncias. Sendo assim, preferimos manter todos os grupos de observações. As Figuras 3.12 e 3.13 apresentam os resíduos de Pearson para os dois sub-modelos. Pode-se notar a presença de resíduos com valores altos fora dos padrões usuais, confirmando a falta de ajuste apontada pelas estatísticas de Hosmer e Lemeshow. 


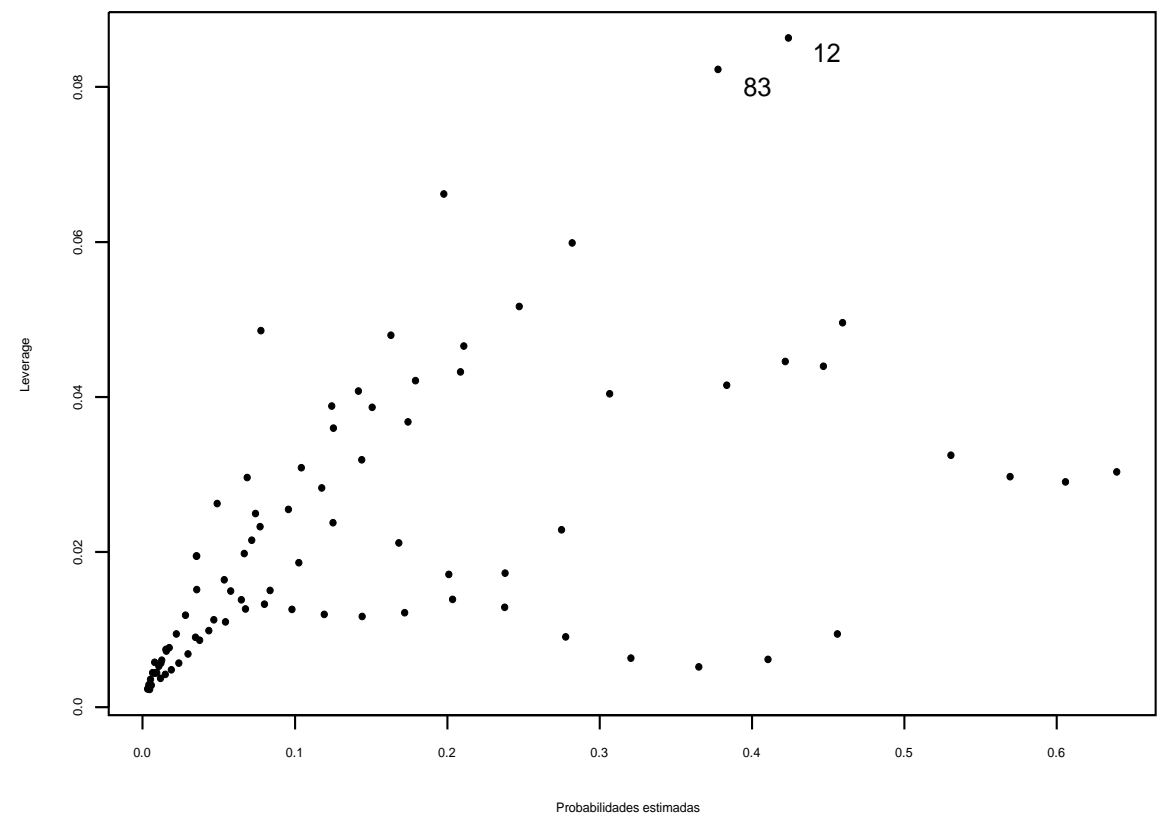

Figura 3.1: Gráfico de $\hat{h}_{i i}^{(1)}$ contra as probabilidades estimadas pelo sub-modelo 1.

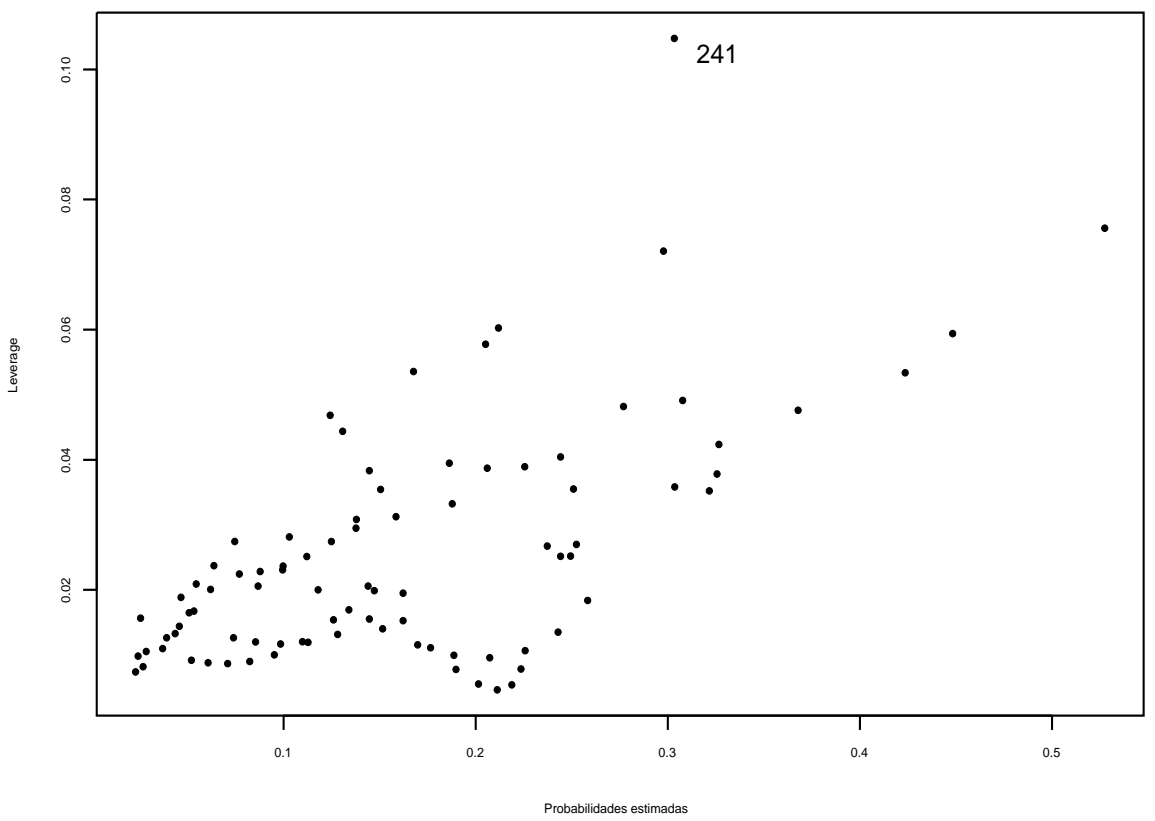

Figura 3.2: Gráfico de $\hat{h}_{i i}^{(2)}$ contra as probabilidades estimadas pelo sub-modelo 2 . 


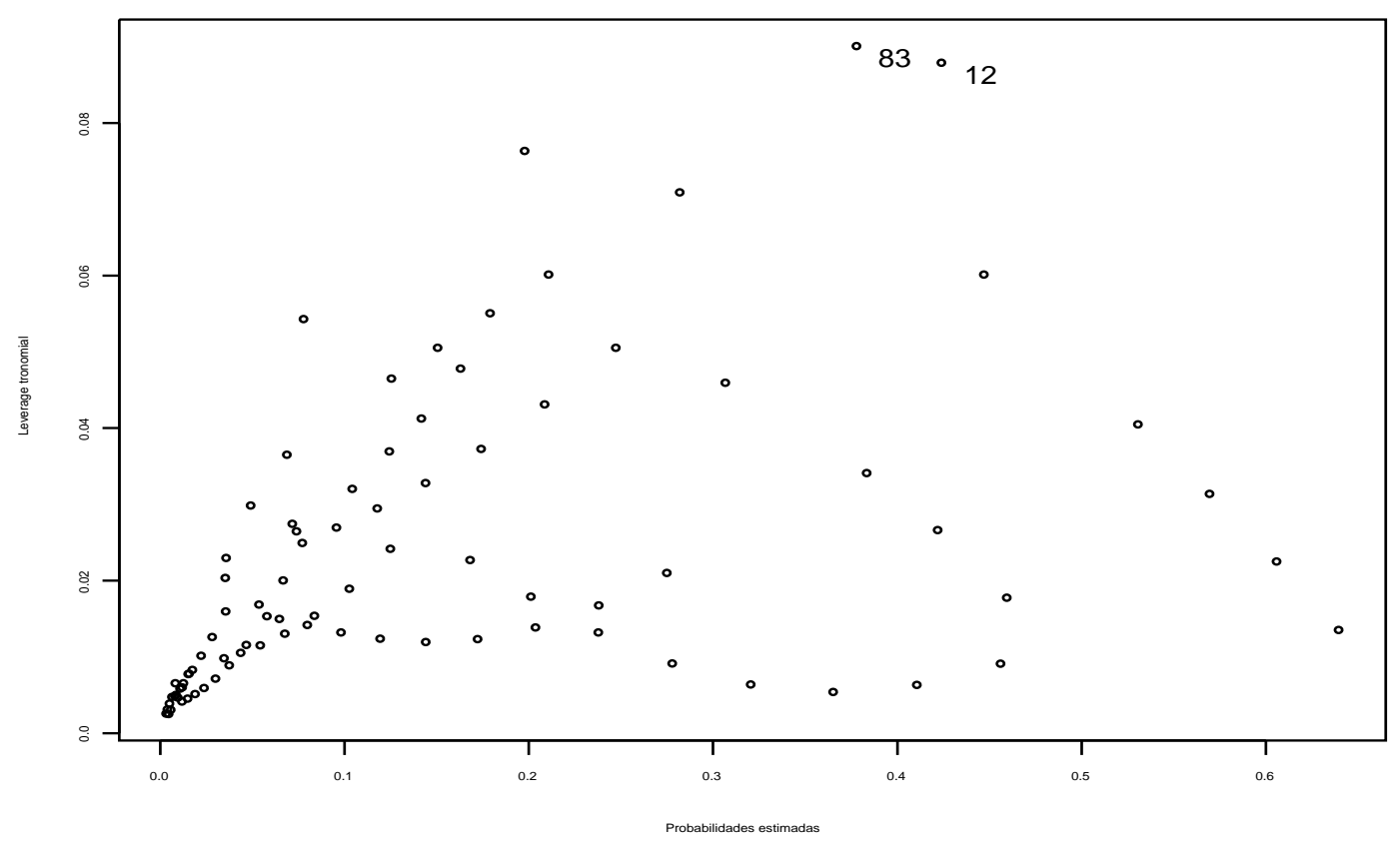

Figura 3.3: Gráfico de $m_{i i}^{(1)}$ contra as probabilidades estimadas do sub-modelo 1.

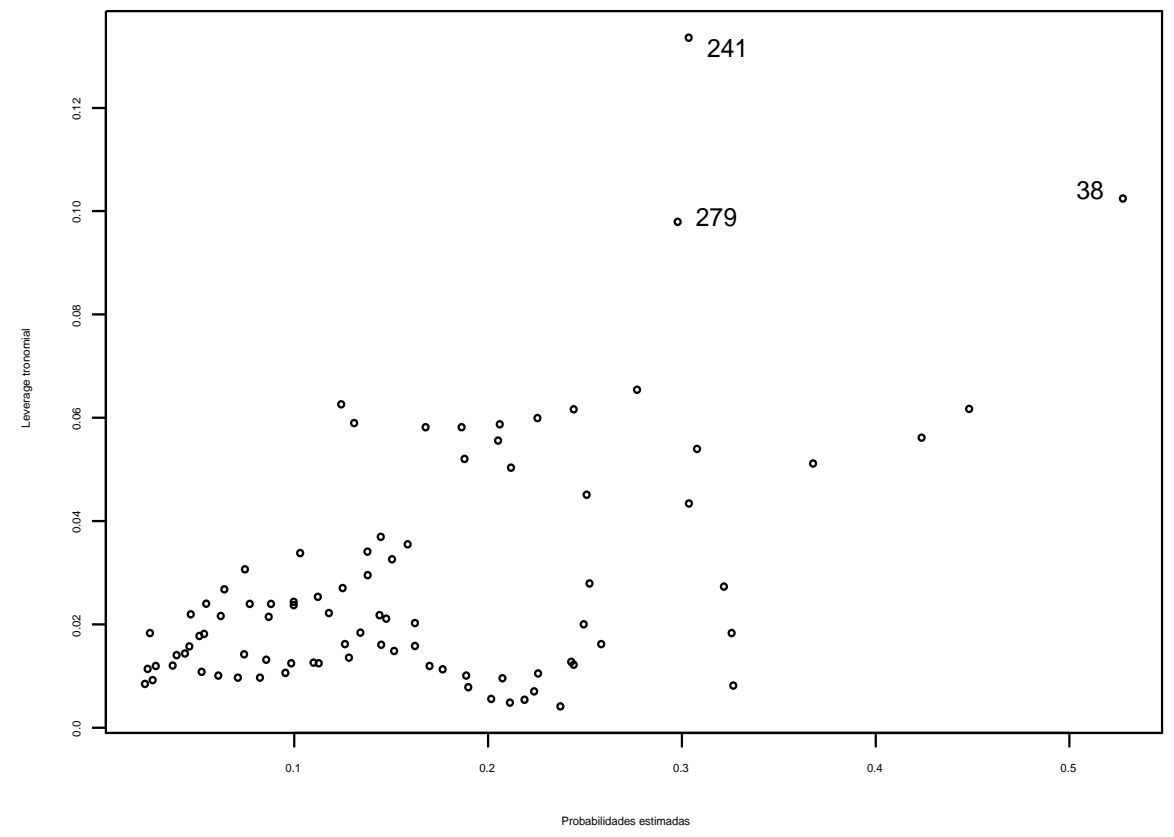

Figura 3.4: Gráfico de $m_{i i}^{(2)}$ contra as probabilidades estimadas do sub-modelo 2. 


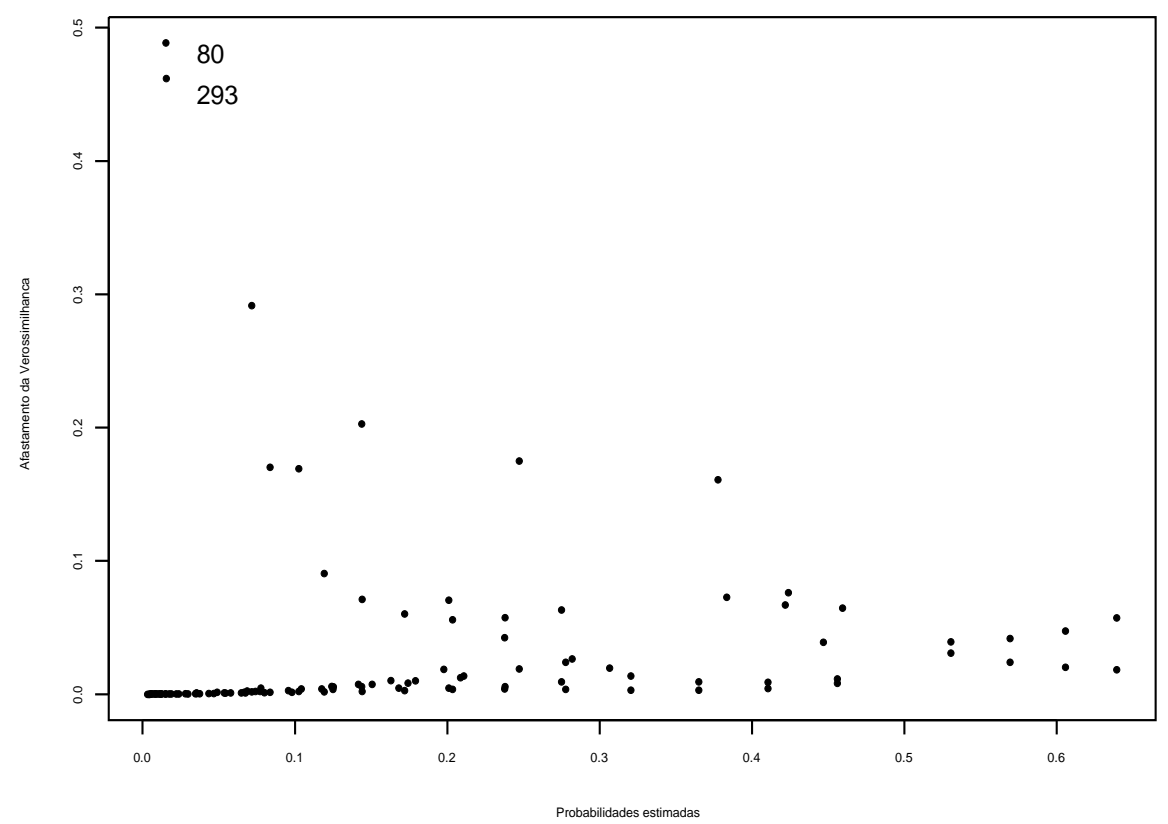

Figura 3.5: Gráfico de $\mathrm{LD}_{i}^{(1)}$ contra as probabilidades estimadas pelo sub-modelo 1.

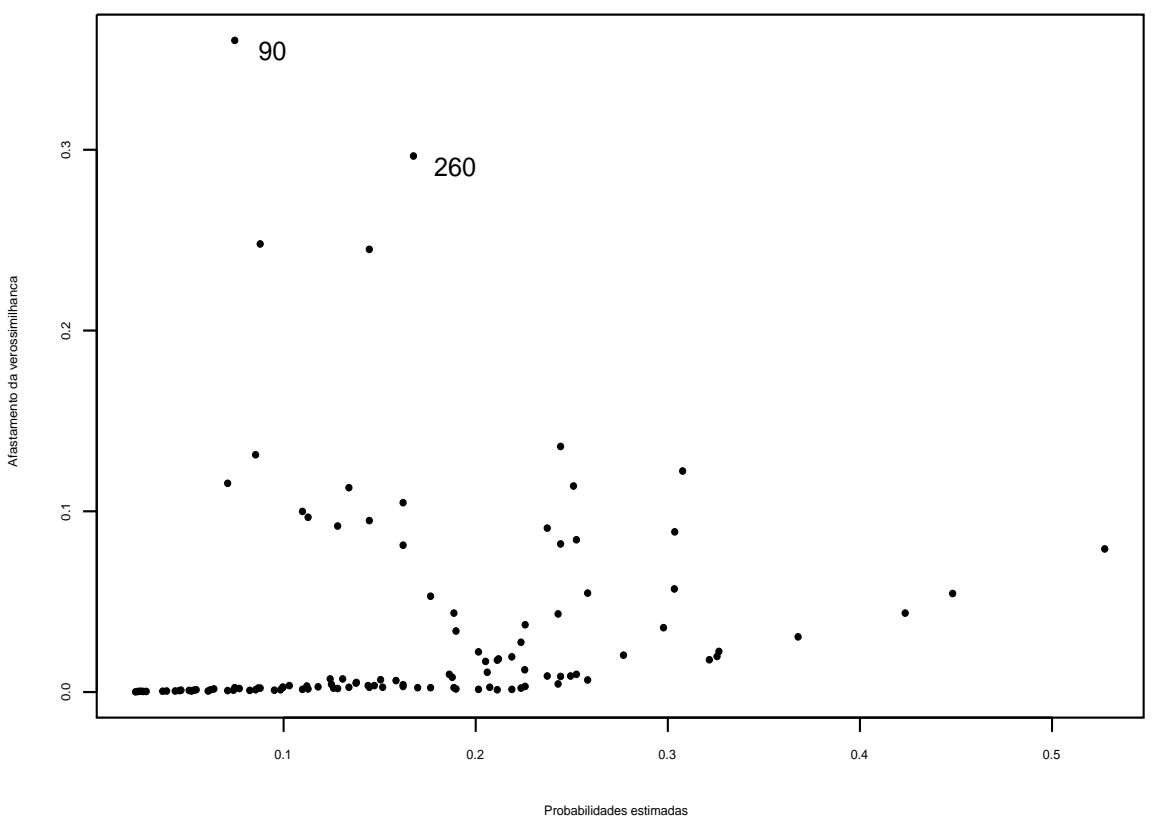

Figura 3.6: Gráfico de $\mathrm{LD}_{i}^{(2)}$ contra as probabilidades estimadas pelo sub-modelo 2. 


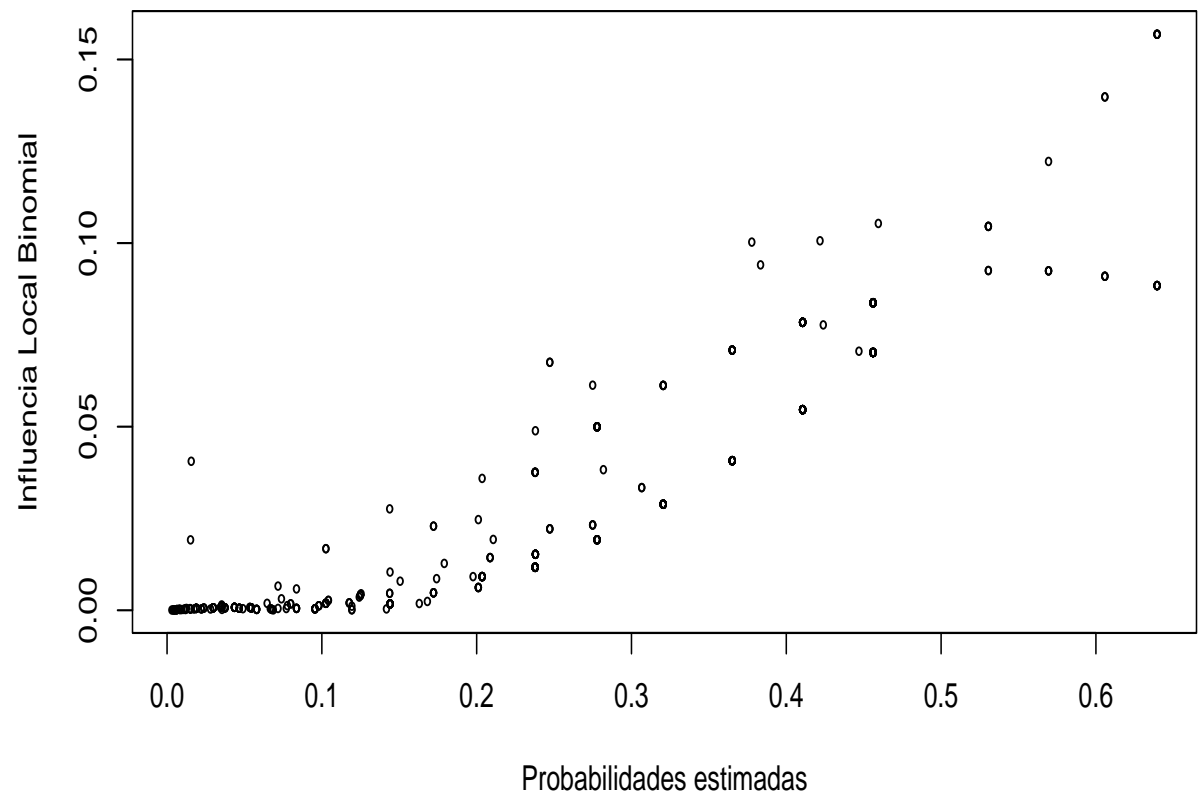

Figura 3.7: Gráfico de $\mathrm{C}_{\ell}^{(1)}$ contra as probabilidades estimadas pelo sub-modelo 1 .

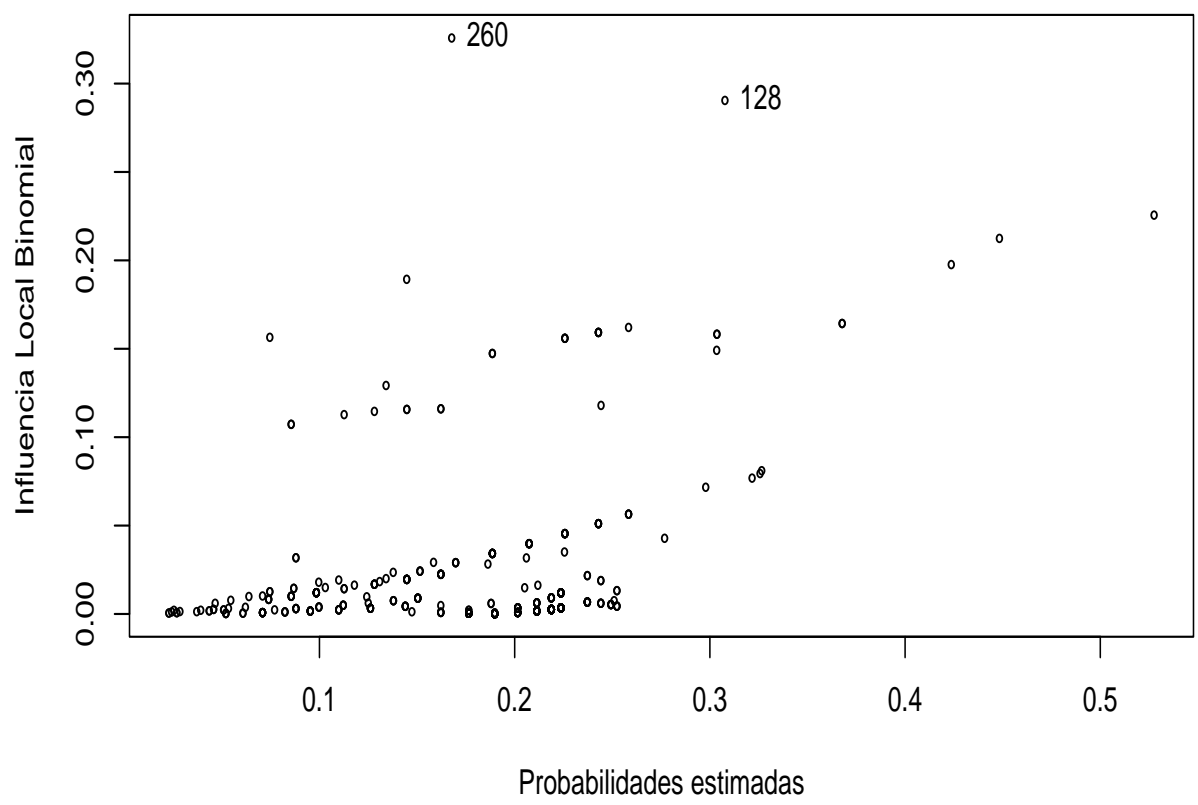

Figura 3.8: Gráfico de $\mathrm{C}_{\ell}^{(2)}$ contra as probabilidades estimadas pelo sub-modelo 2. 


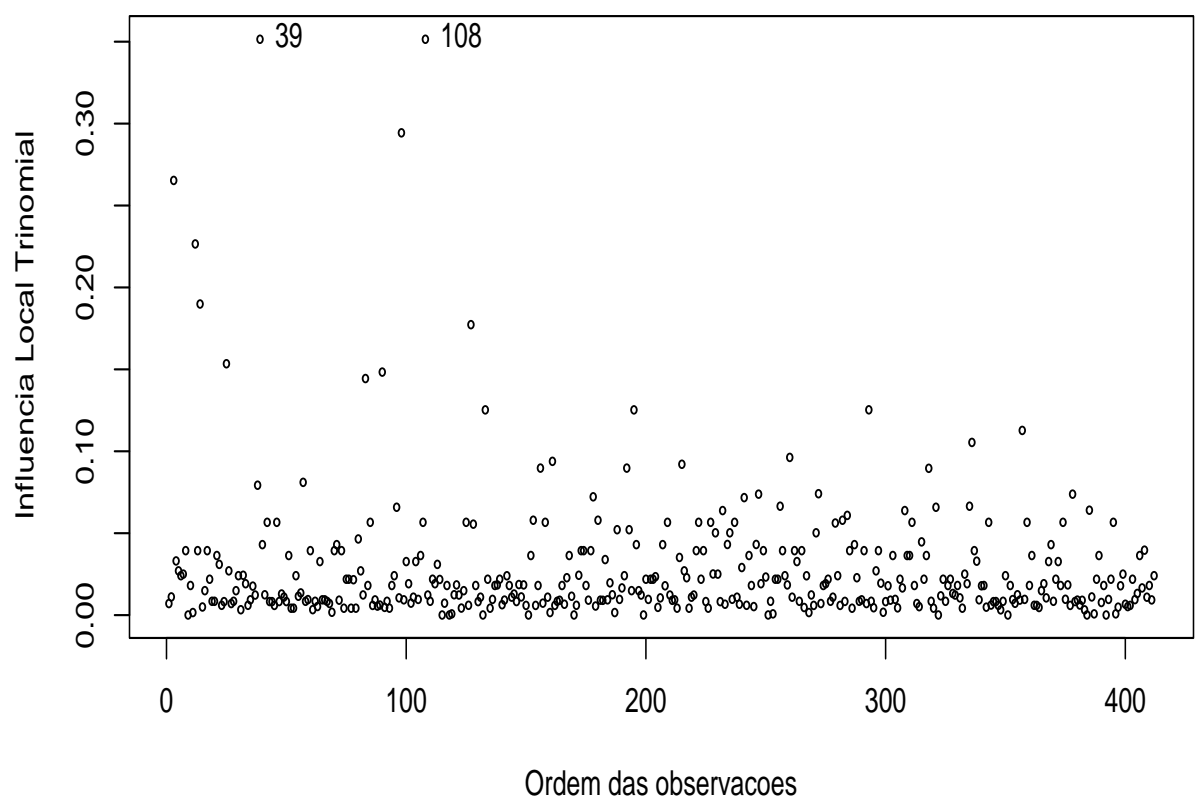

Figura 3.9: Gráfico de $\ell_{\max }$ contra a ordem das observações.

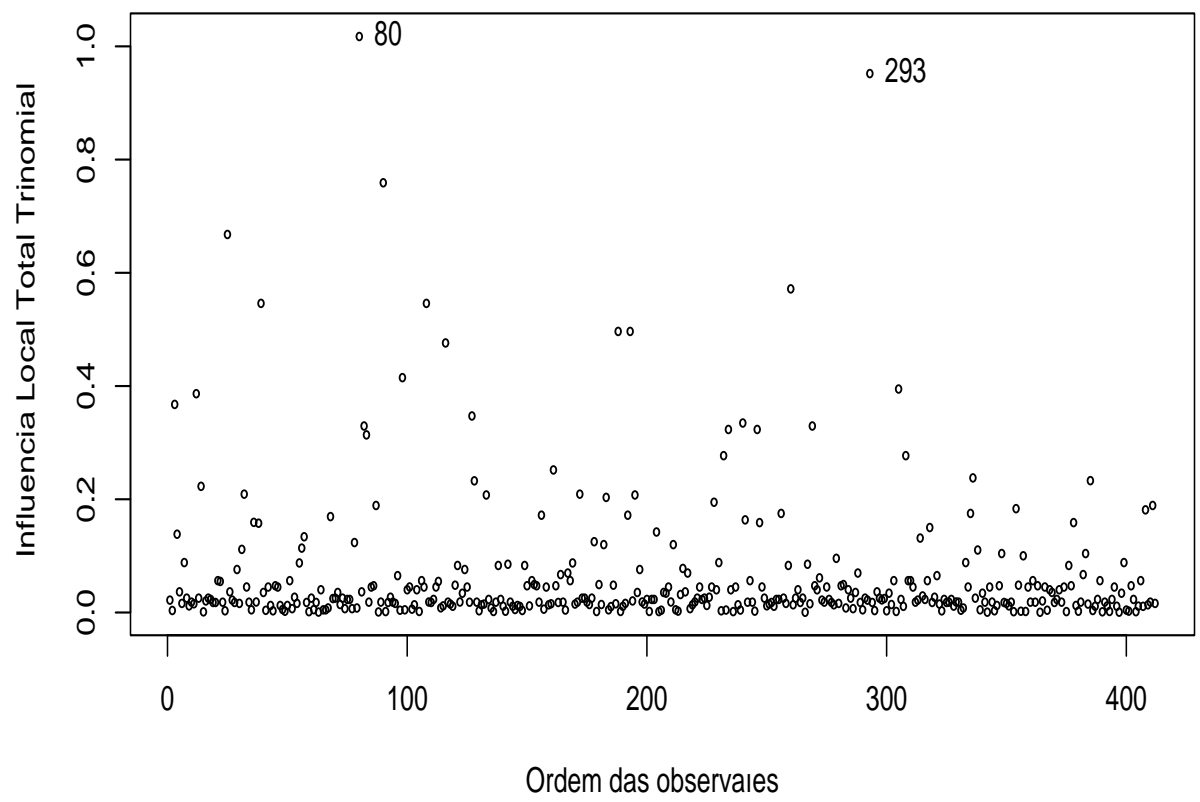

Figura 3.10: Gráfico de $\mathrm{C}_{i}$ contra a ordem das observações. 


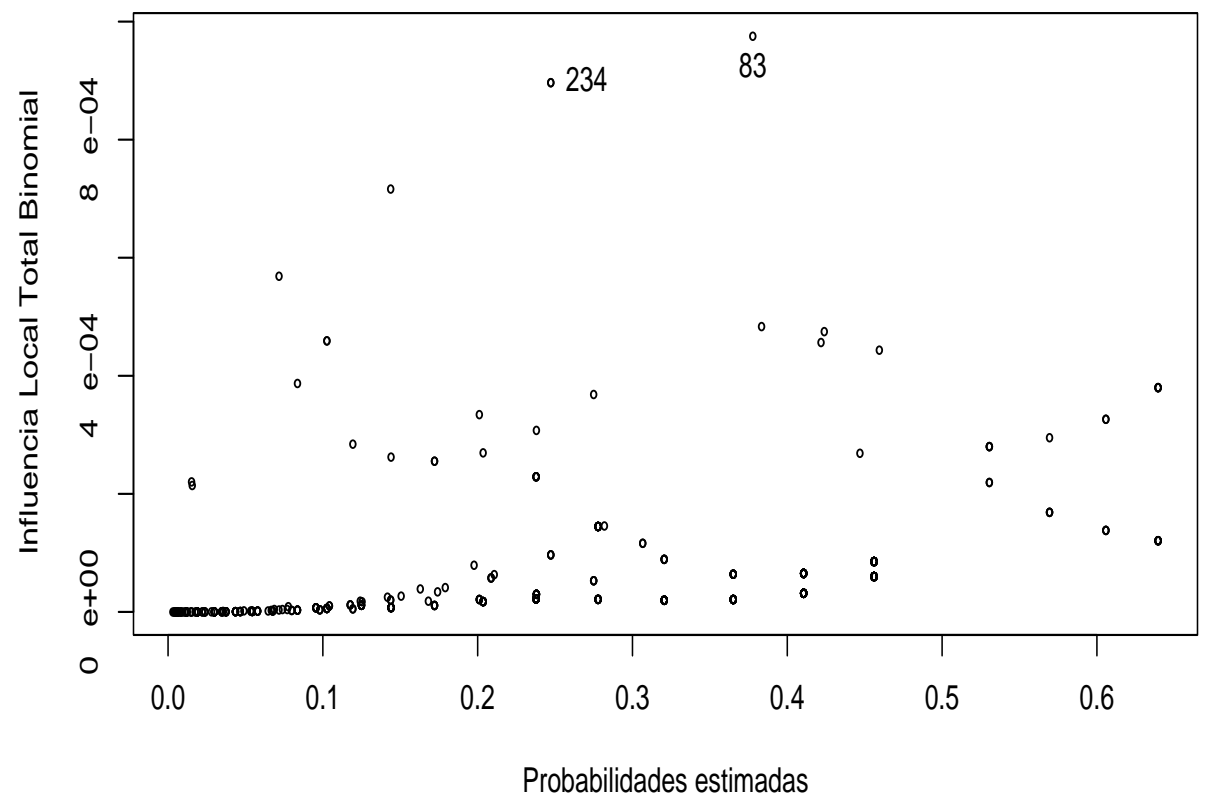

Figura 3.11: Gráfico de $\mathrm{C}_{i}^{(1)}$ contra as probabilidades estimadas pelo sub-modelo 1.

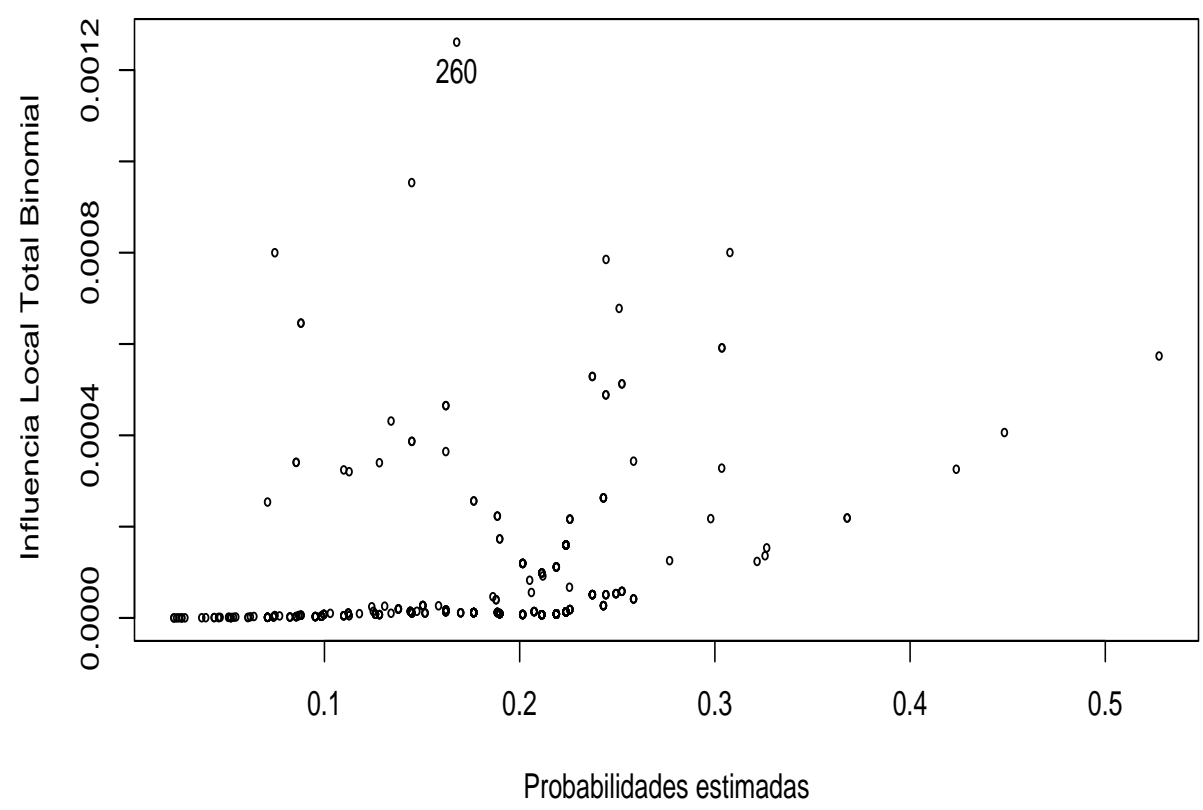

Figura 3.12: Gráfico de $\mathrm{C}_{i}^{(2)}$ contra as probabilidades estimadas pelo sub-modelo 2. 


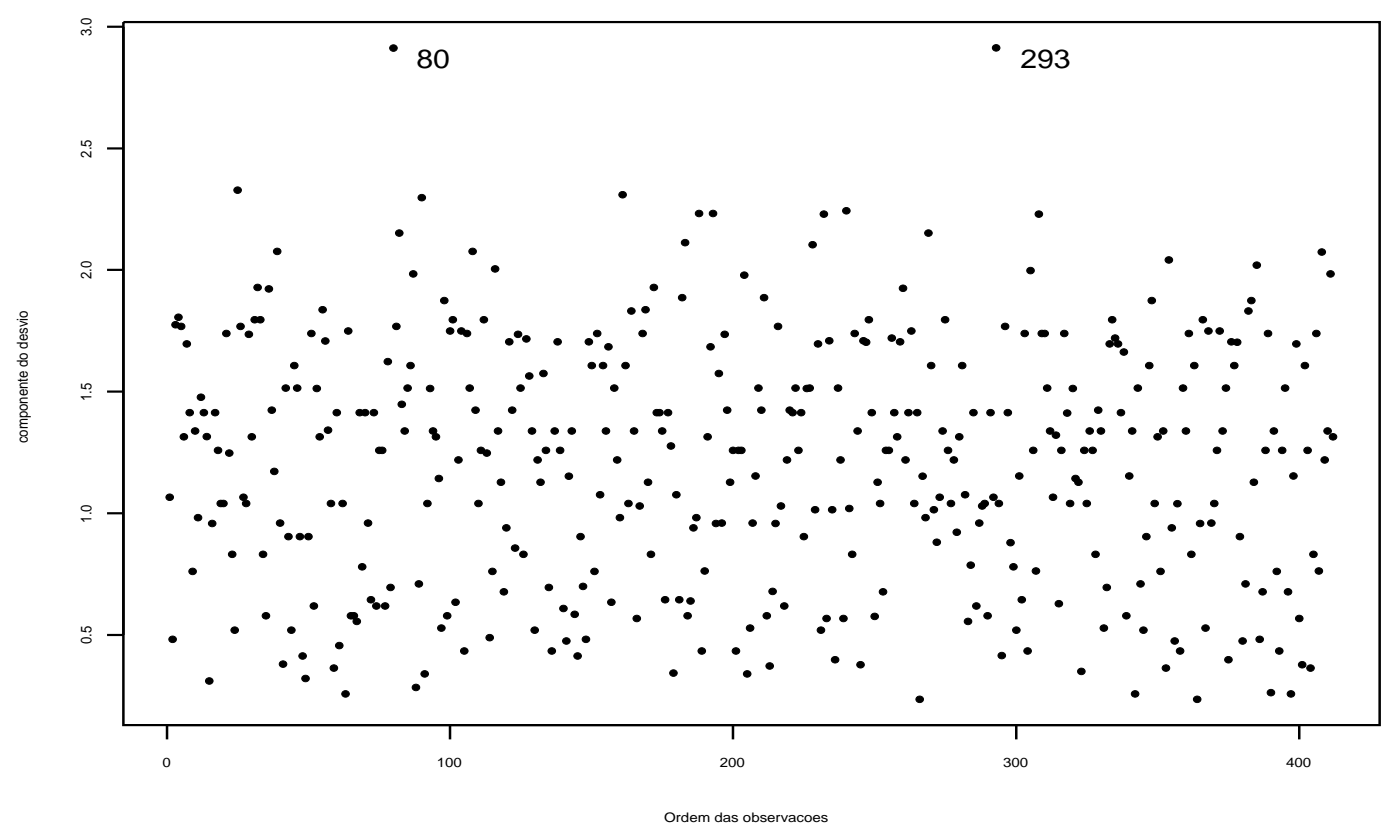

Figura 3.13: Gráfico de $t_{d_{i}}$ contra a ordem das observações.

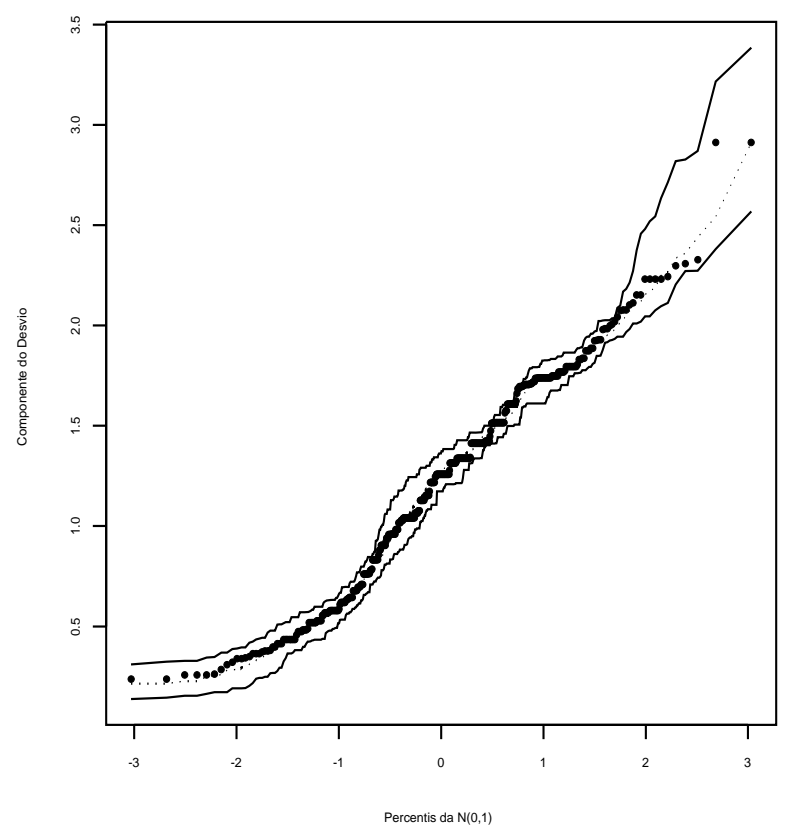

Figura 3.14: Gráfico de envelope $t_{d_{i}}$. 


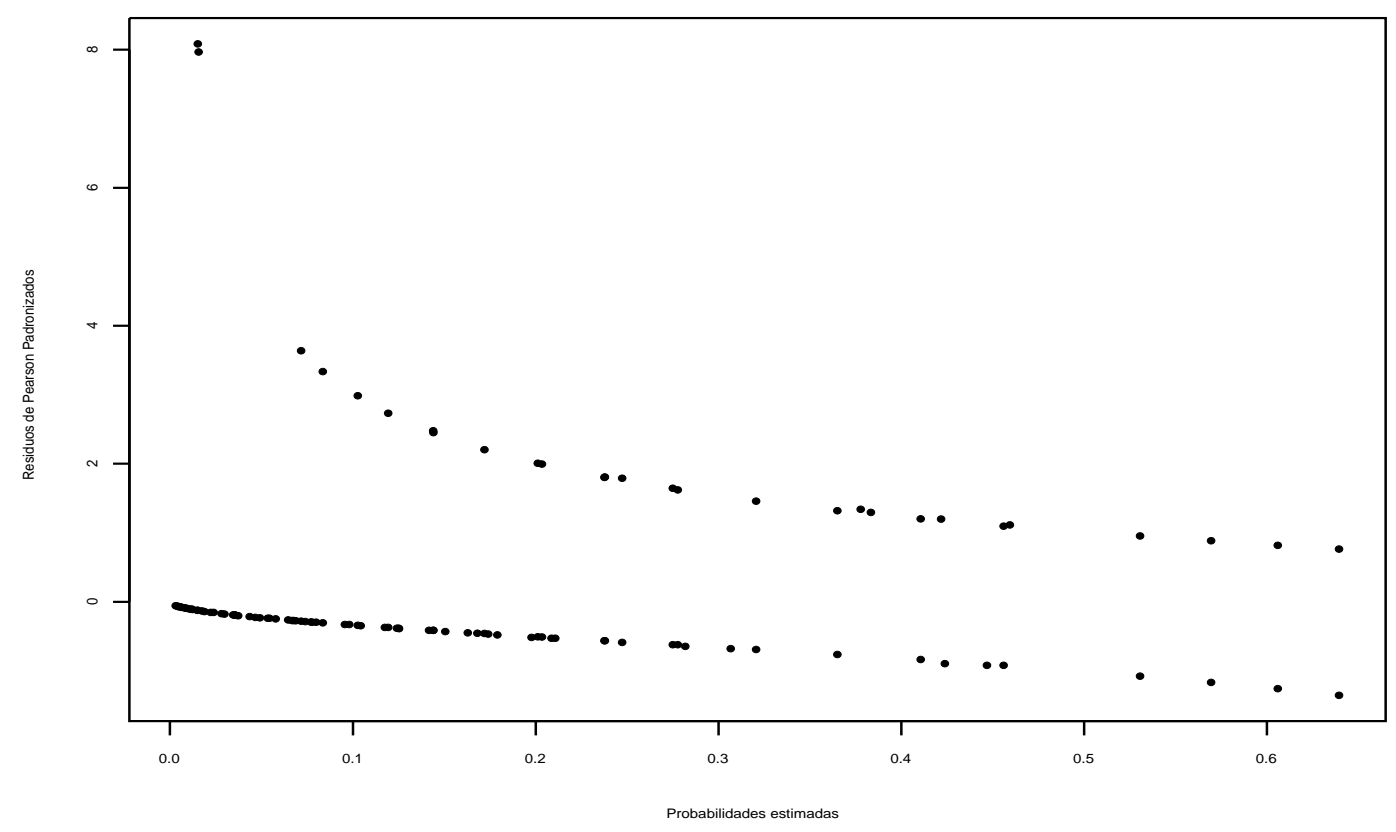

Figura 3.15: Gráfico de $t_{S_{i}}^{(1)}$ contra as probabilidades estimadas pelo sub-modelo 1.

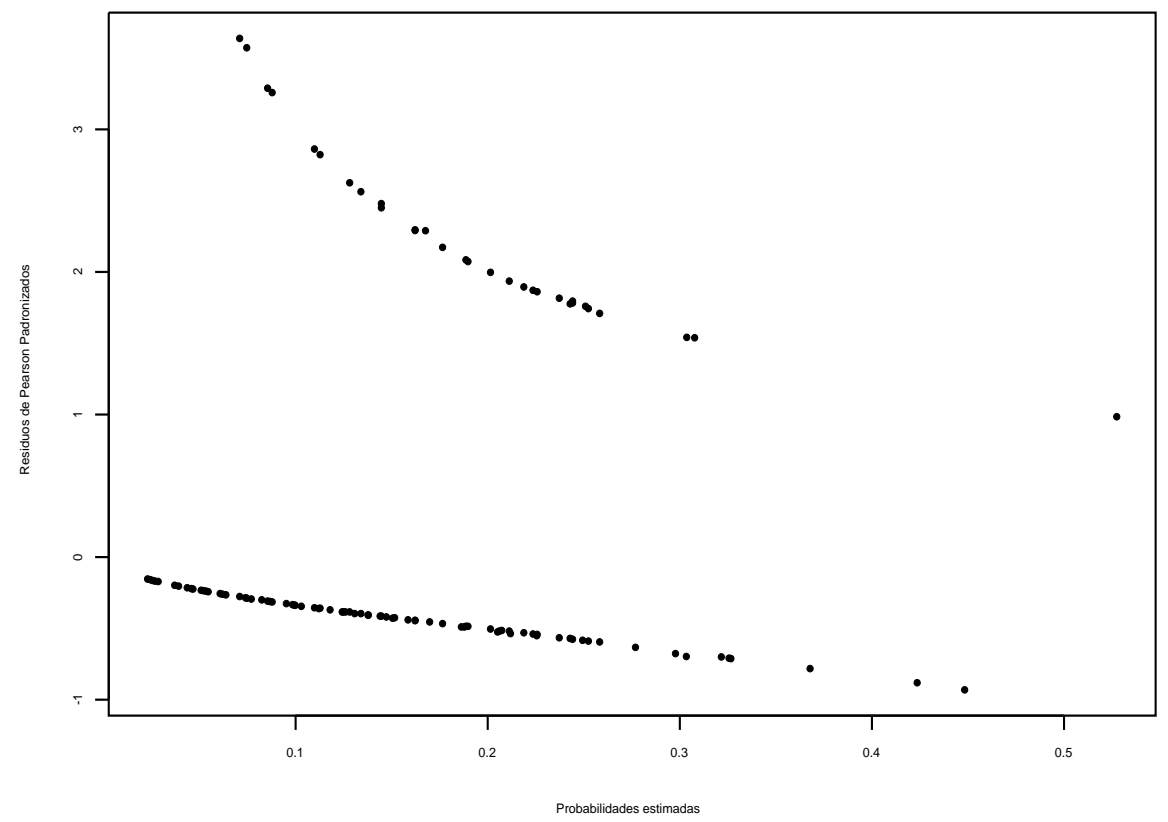

Figura 3.16: Gráfico de $t_{S_{i}}^{(2)}$ contra as probabilidades estimadas pelo sub-modelo 2. 


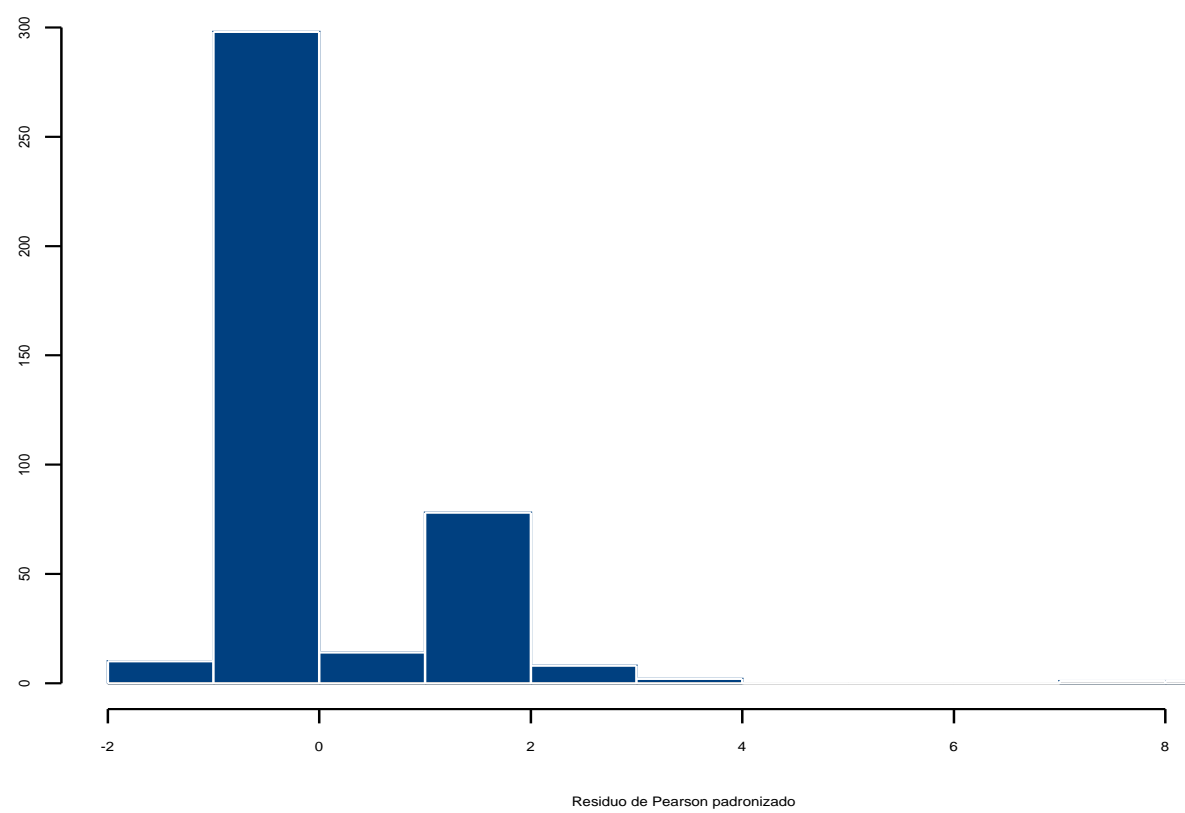

Figura 3.17: Histograma de $t_{S_{i}}^{(1)}$ para o sub-modelo 1.

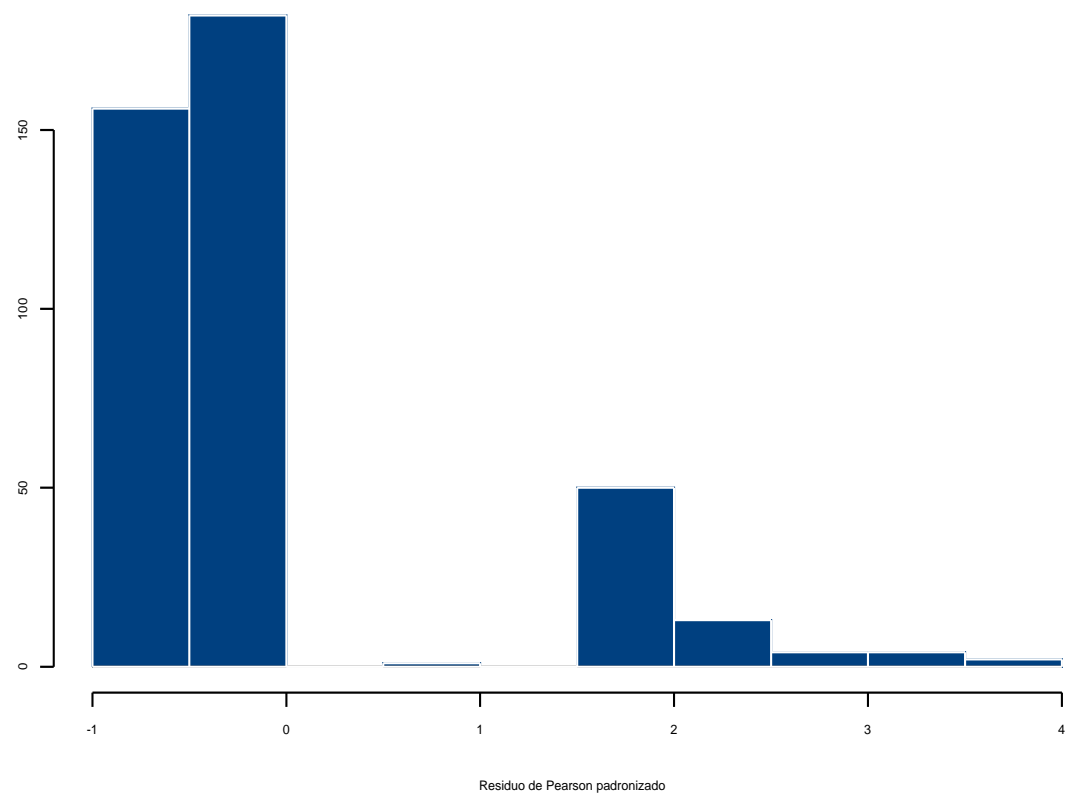

Figura 3.18: Histograma de $t_{S_{i}}^{(2)}$ para o sub-modelo 2. 


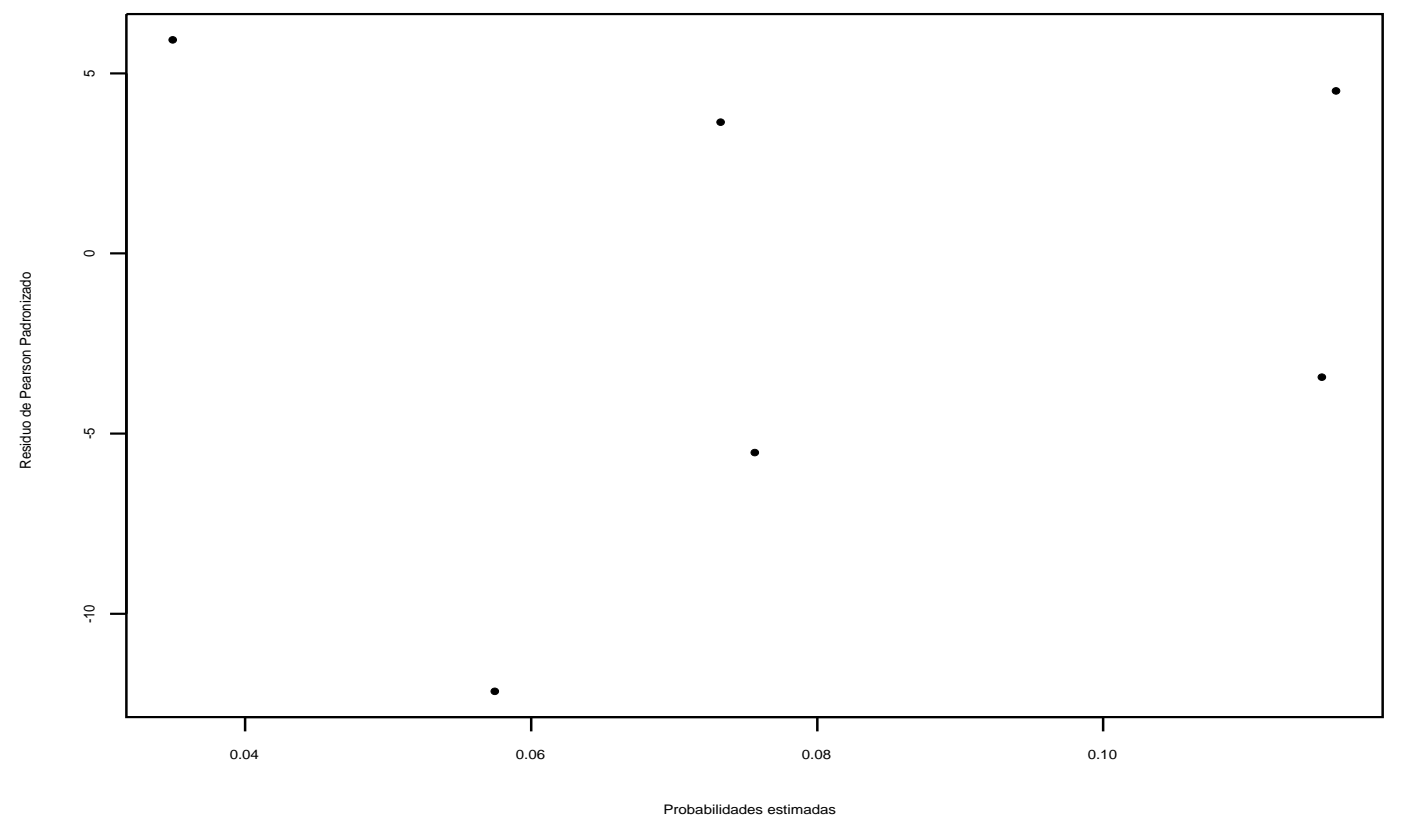

Figura 3.19: Gráfico de $t_{S_{i}}^{(1)}$ contra as probabilidades estimadas pelo sub-modelo 1.

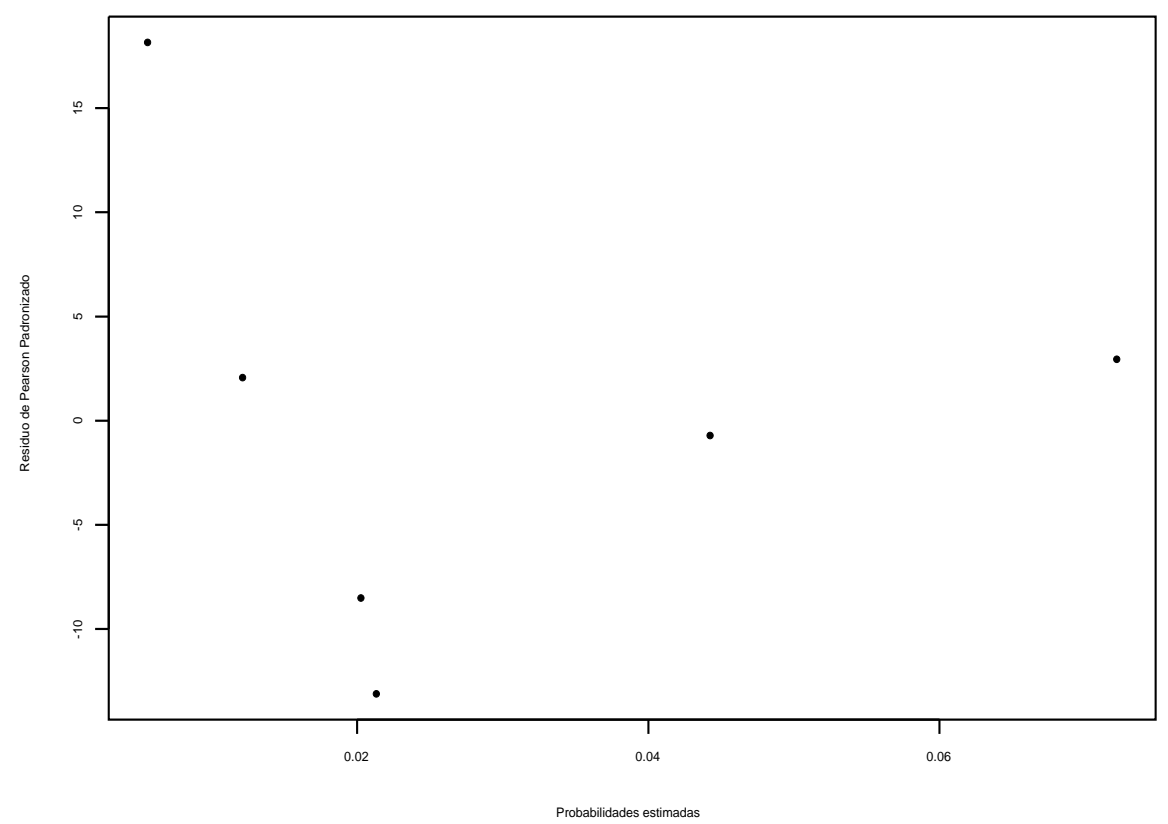

Figura 3.20: Gráfico de $t_{S_{i}}^{(2)}$ contra as probabilidades estimadas pelo sub-modelo 2. 


\section{Capítulo 4}

\section{Considerações Finais}

Neste trabalho procuramos estudar o modelo logístico trinomial que em princípio pode ser olhado como uma extensão natural do modelo logístico binomial. Porém, como foi visto ao longo do texto e principalmente no capítulo 2 onde discutimos os métodos de diagnóstico, as extensões podem ficar bastante complexas do ponto de vista computacional, sugerindo o uso de adaptações do modelo logístico binomial. Essas adaptações montraram-se satisfatórias, como vimos nas análise dos exemplos no capítulo 3.

Contundo, o desenvolvimento de técnicas mais recentes de diagnóstico, tais como influência local e influência local total, vistas no capítulo 2, que aparentemente não parecem tão complicadas como as técnicas de deleção e alavanca, abrem uma perspectiva de trabalhos futuros com aplicações diretas dessas novas técnicas em modelos logísticos trinomiais em substituição às técnicas adaptadas do modelo logístico binomial. 


\section{Apêndice}

\section{Apêndice A}

Tabela 1

Dados do Exemplo 1 descrito na seção 1.9.1.

\begin{tabular}{|c|c|c|c|c|c|c|}
\hline Observação & $\begin{array}{l}\text { Experiência } \\
\text { mamografia }\end{array}$ & Sintoma & $\begin{array}{l}\text { Benefício } \\
\text { percebido }\end{array}$ & $\begin{array}{l}\text { Hitórico } \\
\text { Familiar }\end{array}$ & Autoexame & Detectar \\
\hline 1 & 0 & 3 & 7 & 0 & 1 & 2 \\
\hline 2 & 0 & 2 & 11 & 0 & 1 & 3 \\
\hline 3 & 0 & 3 & 8 & 1 & 1 & 3 \\
\hline 4 & 1 & 3 & 11 & 0 & 1 & 3 \\
\hline 5 & 2 & 4 & 7 & 0 & 1 & 3 \\
\hline 6 & 0 & 3 & 7 & 0 & 1 & 3 \\
\hline 7 & 2 & 4 & 6 & 0 & 1 & 2 \\
\hline 8 & 0 & 4 & 6 & 0 & 1 & 3 \\
\hline 9 & 0 & 2 & 6 & 0 & 1 & 3 \\
\hline 10 & 1 & 4 & 6 & 0 & 1 & 3 \\
\hline 11 & 0 & 4 & 8 & 0 & 1 & 2 \\
\hline 12 & 0 & 3 & 6 & 1 & 0 & 3 \\
\hline 13 & 0 & 4 & 6 & 0 & 1 & 3 \\
\hline 14 & 0 & 1 & 5 & 1 & 1 & 3 \\
\hline 15 & 0 & 2 & 8 & 0 & 0 & 2 \\
\hline 16 & 0 & 4 & 11 & 0 & 1 & 3 \\
\hline 17 & 0 & 3 & 6 & 0 & 1 & 3 \\
\hline 18 & 1 & 4 & 5 & 0 & 1 & 3 \\
\hline 19 & 0 & 3 & 10 & 0 & 1 & 3 \\
\hline 20 & 0 & 3 & 10 & 0 & 1 & 3 \\
\hline 21 & 2 & 3 & 5 & 0 & 1 & 3 \\
\hline 22 & 0 & 4 & 5 & 0 & 1 & 2 \\
\hline 23 & 0 & 1 & 5 & 0 & 1 & 3 \\
\hline 24 & 0 & 2 & $77^{8}$ & 0 & 1 & 2 \\
\hline 25 & 1 & 2 & 9 & 1 & 1 & 2 \\
\hline 26 & 2 & 4 & 7 & 0 & 1 & 3 \\
\hline 27 & 0 & 4 & 7 & 0 & 1 & 2 \\
\hline 28 & 0 & 3 & 10 & 0 & 1 & 3 \\
\hline 29 & 2 & 3 & 7 & 0 & 1 & 2 \\
\hline 30 & 0 & 3 & 7 & 0 & 1 & 3 \\
\hline
\end{tabular}


Tabela 1

Dados do Exemplo 1 descrito na seção 1.9.1.

\begin{tabular}{|c|c|c|c|c|c|c|}
\hline Observação & $\begin{array}{l}\text { Experiência } \\
\text { mamografia }\end{array}$ & Sintoma & $\begin{array}{l}\text { Benefício } \\
\text { percebido }\end{array}$ & $\begin{array}{l}\text { Hitórico } \\
\text { Familiar }\end{array}$ & Autoexame & Detectar \\
\hline 31 & 1 & 4 & 7 & 0 & 1 & 2 \\
\hline 32 & 2 & 2 & 5 & 0 & 1 & 3 \\
\hline 33 & 2 & 3 & 8 & 0 & 1 & 3 \\
\hline 34 & 0 & 1 & 5 & 0 & 1 & 3 \\
\hline 35 & 0 & 2 & 9 & 0 & 1 & 3 \\
\hline 36 & 2 & 3 & 11 & 0 & 1 & 3 \\
\hline 37 & 1 & 3 & 7 & 0 & 1 & 3 \\
\hline 38 & 2 & 3 & 9 & 1 & 1 & 1 \\
\hline 39 & 0 & 3 & 5 & 1 & 1 & 3 \\
\hline 40 & 1 & 3 & 5 & 1 & 1 & 3 \\
\hline 41 & 0 & 2 & 10 & 0 & 0 & 1 \\
\hline 42 & 0 & 4 & 5 & 0 & 1 & 3 \\
\hline 43 & 0 & 3 & 9 & 0 & 1 & 2 \\
\hline 44 & 0 & 2 & 8 & 0 & 1 & 2 \\
\hline 45 & 1 & 3 & 9 & 0 & 1 & 3 \\
\hline 46 & 0 & 3 & 5 & 0 & 1 & 3 \\
\hline 47 & 0 & 3 & 9 & 0 & 1 & 2 \\
\hline 48 & 0 & 2 & 9 & 0 & 0 & 1 \\
\hline 49 & 0 & 1 & 12 & 0 & 0 & 1 \\
\hline 50 & 0 & 4 & 9 & 0 & 1 & 2 \\
\hline 51 & 2 & 4 & 5 & 0 & 1 & 3 \\
\hline 52 & 0 & 2 & 6 & 0 & 1 & 2 \\
\hline 53 & 1 & 4 & 8 & 0 & 1 & 3 \\
\hline 54 & 0 & 4 & 7 & 0 & 1 & 3 \\
\hline 55 & 2 & 3 & 9 & 0 & 1 & 2 \\
\hline 56 & 1 & 4 & 6 & 0 & 1 & 2 \\
\hline 57 & 1 & 4 & 6 & 1 & 1 & 2 \\
\hline 58 & 0 & 3 & 10 & 0 & 1 & 3 \\
\hline 59 & 0 & 2 & 12 & 0 & 1 & 2 \\
\hline 60 & 0 & 4 & 6 & 0 & 1 & 3 \\
\hline 61 & 0 & 1 & 6 & 0 & 0 & 3 \\
\hline 62 & 0 & 3 & 10 & 0 & 1 & 3 \\
\hline 63 & 0 & 2 & 12 & 0 & 0 & 3 \\
\hline 64 & 2 & 4 & 6 & 0 & 1 & 3 \\
\hline 65 & 0 & 2 & 9 & 0 & 1 & 3 \\
\hline 66 & 0 & 2 & 9 & 0 & 1 & 3 \\
\hline 67 & 0 & 3 & 9 & 0 & 0 & 2 \\
\hline 68 & 0 & 4 & 6 & 0 & 1 & 1 \\
\hline 69 & 0 & 3 & 8 & 0 & 0 & 3 \\
\hline 70 & 0 & 3 & 6 & 0 & 1 & 3 \\
\hline
\end{tabular}


Tabela 1

Dados do Exemplo 1 descrito na seção 1.9.1.

\begin{tabular}{|c|c|c|c|c|c|c|}
\hline Observação & $\begin{array}{l}\text { Experiência } \\
\text { mamografia }\end{array}$ & Sintoma & $\begin{array}{l}\text { Benefício } \\
\text { percebido }\end{array}$ & $\begin{array}{l}\text { Hitórico } \\
\text { Familiar }\end{array}$ & Autoexame & Detectar \\
\hline 71 & 1 & 4 & 5 & 1 & 1 & 3 \\
\hline 72 & 0 & 3 & 10 & 0 & 0 & 3 \\
\hline 73 & 0 & 4 & 6 & 0 & 1 & 3 \\
\hline 74 & 0 & 1 & 6 & 0 & 1 & 2 \\
\hline 75 & 1 & 4 & 5 & 0 & 1 & 3 \\
\hline 76 & 1 & 4 & 5 & 0 & 1 & 3 \\
\hline 77 & 0 & 2 & 6 & 0 & 1 & 2 \\
\hline 78 & 1 & 3 & 5 & 0 & 1 & 2 \\
\hline 79 & 0 & 2 & 7 & 0 & 1 & 3 \\
\hline 80 & 1 & 1 & 6 & 0 & 0 & 2 \\
\hline 81 & 2 & 4 & 7 & 0 & 1 & 3 \\
\hline 82 & 1 & 1 & 6 & 0 & 1 & 3 \\
\hline 83 & 1 & 4 & 7 & 1 & 0 & 3 \\
\hline 84 & 1 & 4 & 6 & 0 & 1 & 3 \\
\hline 85 & 0 & 4 & 5 & 0 & 1 & 3 \\
\hline 86 & 1 & 3 & 9 & 0 & 1 & 3 \\
\hline 87 & 2 & 2 & 6 & 0 & 1 & 3 \\
\hline 88 & 0 & 2 & 11 & 0 & 0 & 3 \\
\hline 89 & 0 & 4 & 9 & 0 & 0 & 3 \\
\hline 90 & 2 & 1 & 9 & 0 & 0 & 1 \\
\hline 91 & 0 & 2 & 7 & 0 & 0 & 2 \\
\hline 92 & 0 & 3 & 10 & 0 & 1 & 3 \\
\hline 93 & 1 & 4 & 8 & 0 & 1 & 3 \\
\hline 94 & 1 & 3 & 6 & 0 & 1 & 3 \\
\hline 95 & 0 & 3 & 7 & 0 & 1 & 3 \\
\hline 96 & 1 & 3 & 8 & 1 & 1 & 3 \\
\hline 97 & 0 & 2 & 10 & 0 & 1 & 3 \\
\hline 98 & 0 & 3 & 7 & 1 & 1 & 3 \\
\hline 99 & 0 & 1 & 9 & 0 & 1 & 3 \\
\hline 100 & 2 & 4 & 6 & 0 & 1 & 3 \\
\hline 101 & 2 & 4 & 8 & 0 & 1 & 3 \\
\hline 102 & 0 & 2 & 8 & 0 & 1 & 3 \\
\hline 103 & 0 & 4 & 8 & 0 & 1 & 3 \\
\hline 104 & 2 & 4 & 6 & 0 & 1 & 3 \\
\hline 105 & 0 & 2 & 10 & 0 & 1 & 2 \\
\hline 106 & 2 & 4 & 5 & 0 & 1 & 3 \\
\hline 107 & 0 & 4 & 5 & 0 & 1 & 3 \\
\hline 108 & 0 & 3 & 5 & 1 & 1 & 3 \\
\hline 109 & 1 & 3 & 7 & 0 & 1 & 3 \\
\hline 110 & 0 & 3 & 10 & 0 & 1 & 3 \\
\hline
\end{tabular}


Tabela 1

Dados do Exemplo 1 descrito na seção 1.9.1.

\begin{tabular}{|c|c|c|c|c|c|c|}
\hline Observação & $\begin{array}{l}\text { Experiência } \\
\text { mamografia }\end{array}$ & Sintoma & $\begin{array}{l}\text { Benefício } \\
\text { percebido }\end{array}$ & $\begin{array}{l}\text { Hitórico } \\
\text { Familiar }\end{array}$ & Autoexame & Detectar \\
\hline 111 & 1 & 3 & 5 & 0 & 1 & 3 \\
\hline 112 & 2 & 3 & 8 & 0 & 1 & 3 \\
\hline 113 & 0 & 3 & 5 & 0 & 1 & 2 \\
\hline 114 & 0 & 1 & 13 & 0 & 1 & 1 \\
\hline 115 & 0 & 2 & 6 & 0 & 1 & 3 \\
\hline 116 & 2 & 4 & 5 & 0 & 0 & 2 \\
\hline 117 & 1 & 3 & 6 & 0 & 1 & 3 \\
\hline 118 & 0 & 4 & 9 & 0 & 1 & 3 \\
\hline 119 & 0 & 1 & 5 & 0 & 1 & 2 \\
\hline 120 & 0 & 4 & 6 & 0 & 0 & 3 \\
\hline 121 & 1 & 4 & 10 & 0 & 1 & 3 \\
\hline 122 & 1 & 4 & 7 & 0 & 1 & 3 \\
\hline 123 & 0 & 4 & 7 & 0 & 0 & 3 \\
\hline 124 & 2 & 3 & 7 & 0 & 1 & 2 \\
\hline 125 & 0 & 4 & 5 & 0 & 1 & 3 \\
\hline 126 & 0 & 1 & 5 & 0 & 1 & 3 \\
\hline 127 & 2 & 2 & 7 & 1 & 1 & 3 \\
\hline 128 & 2 & 3 & 11 & 0 & 1 & 1 \\
\hline 129 & 1 & 4 & 6 & 0 & 1 & 3 \\
\hline 130 & 0 & 1 & 8 & 0 & 1 & 2 \\
\hline 131 & 0 & 3 & 8 & 0 & 1 & 3 \\
\hline 132 & 0 & 3 & 9 & 0 & 1 & 3 \\
\hline 133 & 2 & 3 & 9 & 1 & 1 & 2 \\
\hline 134 & 1 & 4 & 5 & 0 & 1 & 3 \\
\hline 135 & 0 & 2 & 7 & 0 & 1 & 3 \\
\hline 136 & 0 & 1 & 10 & 0 & 1 & 2 \\
\hline 137 & 1 & 3 & 6 & 0 & 1 & 3 \\
\hline 138 & 1 & 3 & 10 & 0 & 1 & 3 \\
\hline 139 & 1 & 3 & 5 & 0 & 1 & 3 \\
\hline 140 & 0 & 4 & 8 & 0 & 0 & 2 \\
\hline 141 & 0 & 1 & 9 & 0 & 1 & 2 \\
\hline 142 & 0 & 3 & 9 & 0 & 1 & 1 \\
\hline 143 & 1 & 4 & 6 & 0 & 1 & 3 \\
\hline 144 & 0 & 4 & 11 & 0 & 0 & 3 \\
\hline 145 & 0 & 2 & 9 & 0 & 0 & 1 \\
\hline 146 & 0 & 3 & 9 & 0 & 1 & 2 \\
\hline 147 & 0 & 4 & 12 & 0 & 1 & 2 \\
\hline 148 & 0 & 1 & 11 & 0 & 1 & 3 \\
\hline 149 & 1 & 3 & 10 & 0 & 1 & 3 \\
\hline 150 & 1 & 3 & 9 & 0 & 1 & 3 \\
\hline
\end{tabular}


Tabela 1

Dados do Exemplo 1 descrito na seção 1.9.1.

\begin{tabular}{|c|c|c|c|c|c|c|}
\hline Observação & $\begin{array}{l}\text { Experiência } \\
\text { mamografia }\end{array}$ & Sintoma & $\begin{array}{l}\text { Benefício } \\
\text { percebido }\end{array}$ & $\begin{array}{l}\text { Hitórico } \\
\text { Familiar }\end{array}$ & Autoexame & Detectar \\
\hline 151 & 0 & 2 & 6 & 0 & 1 & 3 \\
\hline 152 & 2 & 3 & 5 & 0 & 1 & 3 \\
\hline 153 & 1 & 3 & 7 & 1 & 1 & 3 \\
\hline 154 & 1 & 3 & 9 & 0 & 1 & 3 \\
\hline 155 & 1 & 4 & 6 & 0 & 1 & 3 \\
\hline 156 & 2 & 3 & 8 & 1 & 1 & 3 \\
\hline 157 & 0 & 1 & 8 & 0 & 1 & 3 \\
\hline 158 & 0 & 4 & 5 & 0 & 1 & 3 \\
\hline 159 & 0 & 3 & 8 & 0 & 1 & 3 \\
\hline 160 & 0 & 4 & 8 & 0 & 1 & 2 \\
\hline 161 & 2 & 1 & 10 & 0 & 1 & 2 \\
\hline 162 & 1 & 4 & 9 & 0 & 1 & 3 \\
\hline 163 & 0 & 4 & 10 & 0 & 1 & 3 \\
\hline 164 & 2 & 4 & 9 & 0 & 1 & 3 \\
\hline 165 & 1 & 4 & 6 & 0 & 1 & 3 \\
\hline 166 & 0 & 1 & 7 & 0 & 1 & 2 \\
\hline 167 & 0 & 3 & 5 & 0 & 0 & 3 \\
\hline 168 & 2 & 3 & 5 & 0 & 1 & 3 \\
\hline 169 & 2 & 4 & 9 & 0 & 1 & 2 \\
\hline 170 & 0 & 3 & 9 & 0 & 1 & 3 \\
\hline 171 & 0 & 1 & 5 & 0 & 1 & 3 \\
\hline 172 & 2 & 1 & 5 & 0 & 1 & 3 \\
\hline 173 & 0 & 4 & 6 & 0 & 1 & 3 \\
\hline 174 & 0 & 3 & 6 & 0 & 1 & 3 \\
\hline 175 & 1 & 4 & 6 & 0 & 1 & 3 \\
\hline 176 & 0 & 3 & 10 & 0 & 0 & 3 \\
\hline 177 & 0 & 4 & 6 & 0 & 1 & 3 \\
\hline 178 & 1 & 3 & 5 & 1 & 1 & 2 \\
\hline 179 & 0 & 2 & 9 & 0 & 0 & 3 \\
\hline 180 & 1 & 3 & 7 & 1 & 1 & 3 \\
\hline 181 & 0 & 3 & 10 & 0 & 0 & 3 \\
\hline 182 & 1 & 3 & 8 & 0 & 1 & 2 \\
\hline 183 & 2 & 2 & 7 & 0 & 1 & 2 \\
\hline 184 & 0 & 2 & 9 & 0 & 1 & 3 \\
\hline 185 & 0 & 3 & 13 & 0 & 1 & 2 \\
\hline 186 & 0 & 4 & 6 & 0 & 0 & 3 \\
\hline 187 & 0 & 3 & 8 & 0 & 1 & 2 \\
\hline 188 & 2 & 3 & 10 & 0 & 0 & 3 \\
\hline 189 & 0 & 2 & 10 & 0 & 1 & 2 \\
\hline 190 & 0 & 4 & 11 & 0 & 1 & 2 \\
\hline
\end{tabular}


Tabela 1

Dados do Exemplo 1 descrito na seção 1.9.1.

\begin{tabular}{|c|c|c|c|c|c|c|}
\hline Observação & $\begin{array}{l}\text { Experiência } \\
\text { mamografia }\end{array}$ & Sintoma & $\begin{array}{l}\text { Benefício } \\
\text { percebido }\end{array}$ & $\begin{array}{l}\text { Hitórico } \\
\text { Familiar }\end{array}$ & Autoexame & Detectar \\
\hline 191 & 0 & 4 & 7 & 0 & 1 & 3 \\
\hline 192 & 2 & 3 & 8 & 1 & 1 & 3 \\
\hline 193 & 2 & 4 & 10 & 0 & 0 & 3 \\
\hline 194 & 0 & 3 & 11 & 0 & 1 & 3 \\
\hline 195 & 2 & 3 & 9 & 1 & 1 & 2 \\
\hline 196 & 1 & 4 & 5 & 1 & 1 & 3 \\
\hline 197 & 2 & 3 & 7 & 0 & 1 & 2 \\
\hline 198 & 1 & 4 & 7 & 0 & 1 & 3 \\
\hline 199 & 0 & 3 & 9 & 0 & 1 & 3 \\
\hline 200 & 1 & 3 & 5 & 0 & 1 & 3 \\
\hline 201 & 0 & 2 & 10 & 0 & 1 & 2 \\
\hline 202 & 1 & 4 & 5 & 0 & 1 & 3 \\
\hline 203 & 1 & 3 & 5 & 0 & 1 & 3 \\
\hline 204 & 1 & 3 & 9 & 0 & 1 & 2 \\
\hline 205 & 0 & 2 & 7 & 0 & 0 & 2 \\
\hline 206 & 0 & 1 & 10 & 0 & 1 & 3 \\
\hline 207 & 1 & 4 & 5 & 1 & 1 & 3 \\
\hline 208 & 0 & 4 & 6 & 0 & 1 & 2 \\
\hline 209 & 0 & 3 & 5 & 0 & 1 & 3 \\
\hline 210 & 1 & 3 & 7 & 0 & 1 & 3 \\
\hline 211 & 1 & 3 & 8 & 0 & 1 & 2 \\
\hline 212 & 0 & 2 & 9 & 0 & 1 & 3 \\
\hline 213 & 0 & 1 & 6 & 0 & 0 & 2 \\
\hline 214 & 0 & 2 & 13 & 1 & 1 & 3 \\
\hline 215 & 0 & 2 & 9 & 1 & 1 & 3 \\
\hline 216 & 2 & 4 & 7 & 0 & 1 & 3 \\
\hline 217 & 0 & 4 & 5 & 0 & 0 & 3 \\
\hline 218 & 0 & 1 & 6 & 0 & 1 & 2 \\
\hline 219 & 0 & 3 & 8 & 0 & 1 & 3 \\
\hline 220 & 1 & 4 & 7 & 0 & 1 & 3 \\
\hline 221 & 0 & 3 & 6 & 0 & 1 & 3 \\
\hline 222 & 0 & 3 & 5 & 0 & 1 & 3 \\
\hline 223 & 1 & 4 & 5 & 0 & 1 & 3 \\
\hline 224 & 0 & 3 & 6 & 0 & 1 & 3 \\
\hline 225 & 0 & 4 & 9 & 0 & 1 & 2 \\
\hline 226 & 1 & 3 & 8 & 0 & 1 & 3 \\
\hline 227 & 0 & 3 & 5 & 0 & 1 & 3 \\
\hline 228 & 2 & 2 & 8 & 0 & 1 & 3 \\
\hline 229 & 1 & 4 & 6 & 1 & 1 & 3 \\
\hline 230 & 2 & 4 & 6 & 0 & 1 & 2 \\
\hline
\end{tabular}


Tabela 1

Dados do Exemplo 1 descrito na seção 1.9.1.

\begin{tabular}{|c|c|c|c|c|c|c|}
\hline Observação & $\begin{array}{l}\text { Experiência } \\
\text { mamografia }\end{array}$ & Sintoma & $\begin{array}{l}\text { Benefício } \\
\text { percebido }\end{array}$ & $\begin{array}{l}\text { Hitórico } \\
\text { Familiar }\end{array}$ & Autoexame & Detectar \\
\hline 231 & 0 & 2 & 8 & 0 & 1 & 2 \\
\hline 232 & 2 & 1 & 10 & 0 & 1 & 3 \\
\hline 233 & 0 & 2 & 7 & 0 & 1 & 2 \\
\hline 234 & 1 & 4 & 5 & 0 & 0 & 3 \\
\hline 235 & 1 & 3 & 6 & 1 & 1 & 3 \\
\hline 236 & 0 & 2 & 11 & 0 & 1 & 2 \\
\hline 237 & 0 & 4 & 5 & 0 & 1 & 3 \\
\hline 238 & 0 & 4 & 8 & 0 & 1 & 3 \\
\hline 239 & 0 & 2 & 7 & 0 & 1 & 2 \\
\hline 240 & 1 & 2 & 7 & 0 & 1 & 3 \\
\hline 241 & 0 & 3 & 11 & 1 & 0 & 1 \\
\hline 242 & 0 & 1 & 5 & 0 & 1 & 3 \\
\hline 243 & 2 & 4 & 5 & 0 & 1 & 3 \\
\hline 244 & 1 & 4 & 6 & 0 & 1 & 3 \\
\hline 245 & 0 & 1 & 8 & 0 & 0 & 3 \\
\hline 246 & 1 & 4 & 5 & 0 & 0 & 3 \\
\hline 247 & 2 & 3 & 6 & 1 & 1 & 3 \\
\hline 248 & 2 & 4 & 8 & 0 & 1 & 3 \\
\hline 249 & 0 & 3 & 6 & 0 & 1 & 3 \\
\hline 250 & 0 & 1 & 11 & 0 & 1 & 1 \\
\hline 251 & 0 & 3 & 9 & 0 & 1 & 3 \\
\hline 252 & 0 & 3 & 10 & 0 & 1 & 3 \\
\hline 253 & 0 & 1 & 5 & 0 & 1 & 2 \\
\hline 254 & 1 & 3 & 5 & 0 & 1 & 3 \\
\hline 255 & 1 & 4 & 5 & 0 & 1 & 3 \\
\hline 256 & 2 & 4 & 5 & 1 & 1 & 3 \\
\hline 257 & 0 & 4 & 6 & 0 & 1 & 3 \\
\hline 258 & 0 & 4 & 7 & 0 & 1 & 3 \\
\hline 259 & 1 & 3 & 10 & 0 & 1 & 3 \\
\hline 260 & 2 & 3 & 10 & 0 & 0 & 1 \\
\hline 261 & 0 & 3 & 8 & 0 & 1 & 3 \\
\hline 262 & 0 & 4 & 6 & 0 & 1 & 3 \\
\hline 263 & 2 & 4 & 6 & 0 & 1 & 3 \\
\hline 264 & 0 & 3 & 10 & 0 & 1 & 3 \\
\hline 265 & 0 & 4 & 6 & 0 & 1 & 3 \\
\hline 266 & 0 & 2 & 11 & 0 & 0 & 2 \\
\hline 267 & 0 & 3 & 9 & 0 & 1 & 1 \\
\hline 268 & 0 & 3 & 8 & 0 & 1 & 2 \\
\hline 269 & 1 & 2 & 6 & 0 & 1 & 3 \\
\hline 270 & 1 & 3 & 9 & 0 & 1 & 3 \\
\hline
\end{tabular}


Tabela 1

Dados do Exemplo 1 descrito na seção 1.9.1.

\begin{tabular}{|c|c|c|c|c|c|c|}
\hline Observação & $\begin{array}{l}\text { Experiência } \\
\text { mamografia }\end{array}$ & Sintoma & $\begin{array}{l}\text { Benefício } \\
\text { percebido }\end{array}$ & $\begin{array}{l}\text { Hitórico } \\
\text { Familiar }\end{array}$ & Autoexame & Detectar \\
\hline 271 & 1 & 3 & 6 & 1 & 1 & 3 \\
\hline 272 & 0 & 2 & 10 & 1 & 1 & 3 \\
\hline 273 & 0 & 3 & 7 & 0 & 1 & 2 \\
\hline 274 & 1 & 3 & 6 & 0 & 1 & 3 \\
\hline 275 & 2 & 3 & 8 & 0 & 1 & 3 \\
\hline 276 & 1 & 4 & 5 & 0 & 1 & 3 \\
\hline 277 & 0 & 3 & 10 & 0 & 1 & 3 \\
\hline 278 & 0 & 4 & 8 & 0 & 1 & 3 \\
\hline 279 & 0 & 2 & 11 & 1 & 1 & 1 \\
\hline 280 & 0 & 4 & 7 & 0 & 1 & 3 \\
\hline 281 & 1 & 3 & 9 & 0 & 1 & 3 \\
\hline 282 & 1 & 4 & 7 & 1 & 1 & 3 \\
\hline 283 & 0 & 3 & 9 & 0 & 0 & 2 \\
\hline 284 & 0 & 2 & 9 & 1 & 1 & 2 \\
\hline 285 & 0 & 3 & 6 & 0 & 1 & 3 \\
\hline 286 & 0 & 2 & 6 & 0 & 1 & 2 \\
\hline 287 & 1 & 4 & 5 & 1 & 1 & 3 \\
\hline 288 & 0 & 3 & 5 & 0 & 0 & 3 \\
\hline 289 & 0 & 4 & 10 & 0 & 1 & 3 \\
\hline 290 & 0 & 2 & 9 & 0 & 1 & 3 \\
\hline 291 & 0 & 3 & 6 & 0 & 1 & 3 \\
\hline 292 & 0 & 3 & 7 & 0 & 1 & 2 \\
\hline 293 & 1 & 2 & 10 & 0 & 1 & 1 \\
\hline 294 & 0 & 3 & 10 & 0 & 1 & 3 \\
\hline 295 & 0 & 1 & 7 & 0 & 0 & 3 \\
\hline 296 & 2 & 4 & 7 & 0 & 1 & 3 \\
\hline 297 & 0 & 4 & 6 & 0 & 1 & 3 \\
\hline 298 & 0 & 3 & 12 & 0 & 1 & 3 \\
\hline 299 & 0 & 3 & 8 & 0 & 0 & 3 \\
\hline 300 & 0 & 2 & 8 & 0 & 1 & 2 \\
\hline 301 & 0 & 4 & 6 & 0 & 1 & 2 \\
\hline 302 & 0 & 3 & 10 & 0 & 0 & 3 \\
\hline 303 & 2 & 3 & 5 & 0 & 1 & 3 \\
\hline 304 & 0 & 2 & 10 & 0 & 1 & 2 \\
\hline 305 & 1 & 4 & 8 & 0 & 0 & 3 \\
\hline 306 & 1 & 4 & 5 & 0 & 1 & 3 \\
\hline 307 & 0 & 4 & 11 & 0 & 1 & 2 \\
\hline 308 & 2 & 2 & 10 & 0 & 1 & 3 \\
\hline 309 & 2 & 3 & 5 & 0 & 1 & 3 \\
\hline 310 & 2 & 3 & 5 & 0 & 1 & 3 \\
\hline
\end{tabular}


Tabela 1

Dados do Exemplo 1 descrito na seção 1.9.1.

\begin{tabular}{|c|c|c|c|c|c|c|}
\hline Observação & $\begin{array}{l}\text { Experiência } \\
\text { mamografia }\end{array}$ & Sintoma & $\begin{array}{l}\text { Benefício } \\
\text { percebido }\end{array}$ & $\begin{array}{l}\text { Hitórico } \\
\text { Familiar }\end{array}$ & Autoexame & Detectar \\
\hline 311 & 0 & 3 & 5 & 0 & 1 & 3 \\
\hline 312 & 1 & 4 & 6 & 0 & 1 & 3 \\
\hline 313 & 0 & 3 & 7 & 0 & 1 & 2 \\
\hline 314 & 0 & 3 & 7 & 0 & 1 & 1 \\
\hline 315 & 0 & 2 & 6 & 1 & 0 & 2 \\
\hline 316 & 1 & 4 & 5 & 0 & 1 & 3 \\
\hline 317 & 2 & 4 & 5 & 0 & 1 & 3 \\
\hline 318 & 1 & 4 & 7 & 1 & 1 & 2 \\
\hline 319 & 0 & 3 & 10 & 0 & 1 & 3 \\
\hline 320 & 1 & 4 & 8 & 0 & 1 & 3 \\
\hline 321 & 1 & 4 & 8 & 1 & 1 & 3 \\
\hline 322 & 0 & 3 & 9 & 0 & 1 & 3 \\
\hline 323 & 0 & 2 & 11 & 0 & 0 & 1 \\
\hline 324 & 1 & 4 & 5 & 0 & 1 & 3 \\
\hline 325 & 0 & 3 & 10 & 0 & 1 & 3 \\
\hline 326 & 1 & 4 & 6 & 0 & 1 & 3 \\
\hline 327 & 1 & 4 & 5 & 0 & 1 & 3 \\
\hline 328 & 0 & 3 & 10 & 0 & 1 & 2 \\
\hline 329 & 1 & 3 & 7 & 0 & 1 & 3 \\
\hline 330 & 1 & 4 & 6 & 0 & 1 & 3 \\
\hline 331 & 0 & 2 & 10 & 0 & 1 & 3 \\
\hline 332 & 0 & 2 & 7 & 0 & 1 & 3 \\
\hline 333 & 2 & 4 & 6 & 0 & 1 & 2 \\
\hline 334 & 2 & 4 & 8 & 0 & 1 & 3 \\
\hline 335 & 2 & 4 & 5 & 1 & 1 & 3 \\
\hline 336 & 2 & 3 & 10 & 1 & 1 & 3 \\
\hline 337 & 0 & 3 & 6 & 0 & 1 & 3 \\
\hline 338 & 2 & 4 & 5 & 0 & 1 & 2 \\
\hline 339 & 0 & 1 & 9 & 0 & 1 & 3 \\
\hline 340 & 0 & 4 & 6 & 0 & 1 & 2 \\
\hline 341 & 1 & 3 & 6 & 0 & 1 & 3 \\
\hline 342 & 0 & 2 & 10 & 0 & 0 & 2 \\
\hline 343 & 0 & 3 & 5 & 0 & 1 & 3 \\
\hline 344 & 0 & 3 & 9 & 0 & 0 & 3 \\
\hline 345 & 0 & 1 & 8 & 0 & 1 & 2 \\
\hline 346 & 0 & 3 & 9 & 0 & 1 & 2 \\
\hline 347 & 1 & 4 & 9 & 0 & 1 & 3 \\
\hline 348 & 2 & 3 & 10 & 0 & 1 & 3 \\
\hline 349 & 0 & 4 & 10 & 0 & 1 & 3 \\
\hline 350 & 0 & 3 & 7 & 0 & 1 & 3 \\
\hline
\end{tabular}


Tabela 1

Dados do Exemplo 1 descrito na seção 1.9.1.

\begin{tabular}{|c|c|c|c|c|c|c|}
\hline Observação & $\begin{array}{l}\text { Experiência } \\
\text { mamografia }\end{array}$ & Sintoma & $\begin{array}{l}\text { Benefício } \\
\text { percebido }\end{array}$ & $\begin{array}{l}\text { Hitórico } \\
\text { Familiar }\end{array}$ & Autoexame & Detectar \\
\hline 351 & 0 & 2 & 6 & 0 & 1 & 3 \\
\hline 352 & 1 & 3 & 6 & 0 & 1 & 3 \\
\hline 353 & 0 & 2 & 12 & 0 & 1 & 2 \\
\hline 354 & 2 & 1 & 7 & 0 & 1 & 3 \\
\hline 355 & 0 & 4 & 6 & 0 & 0 & 3 \\
\hline 356 & 0 & 1 & 9 & 0 & 1 & 2 \\
\hline 357 & 0 & 2 & 8 & 1 & 1 & 3 \\
\hline 358 & 0 & 2 & 10 & 0 & 1 & 2 \\
\hline 359 & 0 & 4 & 5 & 0 & 1 & 3 \\
\hline 360 & 1 & 3 & 6 & 0 & 1 & 3 \\
\hline 361 & 2 & 3 & 5 & 0 & 1 & 3 \\
\hline 362 & 0 & 2 & 5 & 0 & 1 & 3 \\
\hline 363 & 1 & 4 & 9 & 0 & 1 & 3 \\
\hline 364 & 0 & 1 & 11 & 0 & 0 & 2 \\
\hline 365 & 0 & 3 & 11 & 0 & 1 & 3 \\
\hline 366 & 2 & 4 & 8 & 0 & 1 & 3 \\
\hline 367 & 0 & 1 & 10 & 0 & 1 & 3 \\
\hline 368 & 2 & 4 & 6 & 0 & 1 & 3 \\
\hline 369 & 1 & 4 & 5 & 1 & 1 & 3 \\
\hline 370 & 0 & 4 & 10 & 0 & 1 & 3 \\
\hline 371 & 1 & 4 & 5 & 0 & 1 & 3 \\
\hline 372 & 2 & 4 & 6 & 0 & 1 & 3 \\
\hline 373 & 1 & 4 & 6 & 0 & 1 & 3 \\
\hline 374 & 0 & 3 & 5 & 0 & 1 & 3 \\
\hline 375 & 0 & 2 & 11 & 0 & 1 & 2 \\
\hline 376 & 1 & 3 & 10 & 0 & 1 & 3 \\
\hline 377 & 1 & 4 & 9 & 0 & 1 & 3 \\
\hline 378 & 2 & 3 & 6 & 1 & 1 & 3 \\
\hline 379 & 0 & 3 & 9 & 0 & 1 & 2 \\
\hline 380 & 0 & 2 & 9 & 0 & 1 & 2 \\
\hline 381 & 0 & 3 & 9 & 0 & 0 & 3 \\
\hline 382 & 2 & 3 & 9 & 0 & 1 & 3 \\
\hline 383 & 2 & 3 & 10 & 0 & 1 & 3 \\
\hline 384 & 0 & 3 & 9 & 0 & 1 & 3 \\
\hline 385 & 2 & 3 & 12 & 0 & 1 & 2 \\
\hline 386 & 0 & 1 & 11 & 0 & 1 & 3 \\
\hline 387 & 0 & 1 & 5 & 0 & 1 & 2 \\
\hline 388 & 1 & 4 & 5 & 0 & 1 & 3 \\
\hline 389 & 2 & 4 & 5 & 0 & 1 & 3 \\
\hline 390 & 0 & 3 & 17 & 0 & 0 & 2 \\
\hline
\end{tabular}


Tabela 1

Dados do Exemplo 1 descrito na seção 1.9.1.

\begin{tabular}{|c|c|c|c|c|c|c|}
\hline Observação & $\begin{array}{l}\text { Experiência } \\
\text { mamografia }\end{array}$ & Sintoma & $\begin{array}{l}\text { Benefício } \\
\text { percebido }\end{array}$ & $\begin{array}{l}\text { Hitórico } \\
\text { Familiar }\end{array}$ & Autoexame & Detectar \\
\hline 391 & 1 & 3 & 6 & 0 & 1 & 3 \\
\hline 392 & 0 & 2 & 6 & 0 & 1 & 3 \\
\hline 393 & 0 & 2 & 10 & 0 & 1 & 2 \\
\hline 394 & 1 & 3 & 5 & 0 & 1 & 3 \\
\hline 395 & 0 & 3 & 5 & 0 & 1 & 3 \\
\hline 396 & 0 & 1 & 5 & 0 & 1 & 2 \\
\hline 397 & 0 & 2 & 10 & 0 & 0 & 2 \\
\hline 398 & 0 & 4 & 6 & 0 & 1 & 2 \\
\hline 399 & 2 & 3 & 6 & 0 & 1 & 2 \\
\hline 400 & 0 & 2 & 7 & 0 & 1 & 2 \\
\hline 401 & 0 & 2 & 8 & 0 & 0 & 3 \\
\hline 402 & 1 & 3 & 9 & 0 & 1 & 3 \\
\hline 403 & 1 & 4 & 5 & 0 & 1 & 3 \\
\hline 404 & 0 & 2 & 12 & 0 & 1 & 2 \\
\hline 405 & 0 & 3 & 10 & 0 & 1 & 2 \\
\hline 406 & 2 & 3 & 5 & 0 & 1 & 3 \\
\hline 407 & 0 & 3 & 11 & 0 & 1 & 2 \\
\hline 408 & 1 & 3 & 10 & 0 & 1 & 2 \\
\hline 409 & 0 & 4 & 8 & 0 & 1 & 3 \\
\hline 410 & 1 & 4 & 6 & 0 & 1 & 3 \\
\hline 411 & 2 & 2 & 6 & 0 & 1 & 3 \\
\hline 412 & 0 & 4 & 7 & 0 & 1 & 3 \\
\hline
\end{tabular}


Tabela 2

Dados do Eemplo 2 descrito na seção 1.9.2.

\begin{tabular}{llcccc}
\hline \hline $\begin{array}{l}\text { Idade } \\
\text { em anos }\end{array}$ & Status & Normal & $\begin{array}{c}\text { Teste } \\
\text { Indefinido }\end{array}$ & Anormal & Total \\
\hline menos de 40 & Nunca fumou & 577 & 27 & 7 & 611 \\
menos de 40 & Ex-fumante & 192 & 20 & 3 & 215 \\
menos de 40 & Fumante & 682 & 46 & 11 & 739 \\
& & & & & \\
$40-59$ & Nunca fumou & 164 & 4 & 0 & 168 \\
$40-59$ & Ex-fumante & 145 & 15 & 7 & 167 \\
$40-59$ & Fumante & 245 & 47 & 27 & 319 \\
\hline \hline
\end{tabular}

\section{Apêndice B}

Neste apêndice apresentamos os programas em S-plus desenvolvidos para geração dos gráficos do exemplo 1 exibidos no trabalho.

Os sub-modelos foram gerados pelo programa SAS, uma vez que o S-plus não funciona para para modelos trinomias.

Passo1.

Geração do sub-modelos e estimação das probabilidades - feito no SAS

Passo 2.

Gerão dos gráficos. De posse das estimativas das probabilidades estimadas para os dois sub-modelos, partimos para geração dos gráficos.

Passo 3.

Leitura dos dados.

Matriz planejamento, respostas e probabilidades estimadas

graficos.dat $=$ scan(" data.dat", what $=\operatorname{list}(\mathrm{um}=0, \mathrm{x} 1=0, \mathrm{x} 2=0, \mathrm{x} 3=0, \mathrm{x} 4=0, \mathrm{x} 5=0, \mathrm{y} 0=0, \mathrm{y} 1=0, \mathrm{y} 2=0, \mathrm{pi} 0=0, \mathrm{pi} 1=0, \mathrm{pi} 2=0))$ 
attach(graficos.dat)

$\mathrm{X}=\operatorname{cbind}(\mathrm{um}, \mathrm{x} 1, \mathrm{x} 2, \mathrm{x} 3, \mathrm{x} 4, \mathrm{x} 5)$

Grafico de Alavanca - binomial

$\mathrm{v} 1=\mathrm{pi}^{*}(1-\mathrm{pi} 1)$

$\mathrm{w} 1=\operatorname{diag}(\mathrm{v} 1)$

$\mathrm{H} 1=\operatorname{sqrt}(\mathrm{w} 1) \% * \% \mathrm{X} \% * \% \operatorname{solve}(\mathrm{t}(\mathrm{X}) \% * \% \mathrm{w} 1 \% * \% \mathrm{X}) \% * \% \mathrm{t}(\mathrm{X}) \% * \% \operatorname{sqrt}(\mathrm{w} 1)$

$\operatorname{hii1}=\operatorname{diag}(\mathrm{H} 1)$

Gráfico de Alavanca - trinomial

zero $=\operatorname{matrix}(\mathrm{c}(0), \mathrm{nrow}=412, \mathrm{ncol}=6)$

$\mathrm{s} 1=\operatorname{cbind}(\mathrm{X}, \mathrm{zero})$

$\mathrm{s} 2=\operatorname{cbind}($ zero, $\mathrm{X})$

sgrande $=\operatorname{rbind}(\mathrm{s} 1, \mathrm{~s} 2)$

$\mathrm{v} 1=\mathrm{pi}^{*}(1-\mathrm{pi} 1)$

$\mathrm{v} 2=\mathrm{pi} 2 *(1-\mathrm{pi} 2)$

$\mathrm{v} 3=-\mathrm{pi} 1 * \mathrm{p} 12$

$\mathrm{v} 1 \mathrm{~d}=\operatorname{diag}(\mathrm{v} 1)$

$\mathrm{v} 2 \mathrm{~d}=\operatorname{diag}(\mathrm{v} 2)$

$\mathrm{v} 3 \mathrm{~d}=\operatorname{diag}(\mathrm{v} 3)$

$\operatorname{vint} 1=\operatorname{cbind}(\mathrm{v} 1 \mathrm{~d}, \mathrm{v} 3 \mathrm{~d})$

vint $2=\operatorname{cbind}(\mathrm{v} 3 \mathrm{~d}, \mathrm{v} 2 \mathrm{~d})$

vgrande $=\operatorname{rbind}(\operatorname{vint} 1, \operatorname{vint} 2)$

mgrande $=$ sgrande $\% * \%$

solve(t(sgrande) $\% * \%$ vgrande $\% * \%$ sgrande $) \% * \%$ t (sgrande) $\% * \%$ vgrande

mii $=\operatorname{diag}($ mgrande $)$

mii1=array $(, 412)$ 
$\mathrm{k}=1$

while $(\mathrm{k} \leq 412)$

$\operatorname{mii} 1[\mathrm{k}]=\operatorname{mii}[\mathrm{k}]$

$\mathrm{k}=\mathrm{k}+1$

Gráfico Afastamento da verossimilhança - binomial

res1=y1-pi1

$\operatorname{corrp} 1=\operatorname{sqrt}($ pi1 $*(1-$ pi1 $) *(1-h i i 1))$

$\mathrm{tS} 1=$ res1/corrp1

$\mathrm{LDi1}=($ hii1 $/(1-\mathrm{hii1})) *(\mathrm{tS} 1 * \mathrm{tS} 1)$

Gráfico Influencia local - trinomial

$\mathrm{v} 1=\mathrm{pi}^{*}(1-\mathrm{pi} 1)$

$\mathrm{v} 2=$ pi $2 *(1-$ pi 2$)$

$\mathrm{v} 3=-\mathrm{pi} 1^{*} \mathrm{p} 12$

$\mathrm{v} 1 \mathrm{~d}=\operatorname{diag}(\mathrm{v} 1)$

$\mathrm{v} 2 \mathrm{~d}=\operatorname{diag}(\mathrm{v} 2)$

$\mathrm{v} 3 \mathrm{~d}=\operatorname{diag}(\mathrm{v} 3)$

$\operatorname{vint} 1=\operatorname{cbind}(\mathrm{v} 1 \mathrm{~d}, \mathrm{v} 3 \mathrm{~d})$

$\operatorname{vint} 2=\operatorname{cbind}(\mathrm{v} 3 \mathrm{~d}, \mathrm{v} 2 \mathrm{~d})$

vzona $=$ rbind $($ vint 1, vint2)

zero $=\operatorname{matrix}(\mathrm{c}(0), \mathrm{nrow}=412, \mathrm{ncol}=6)$

$\mathrm{s} 1=\operatorname{cbind}(\mathrm{X}, \mathrm{zero})$

$\mathrm{s} 2=\operatorname{cbind}($ zero, $\mathrm{X})$

szona $=\operatorname{rbind}(\mathrm{s} 1, \mathrm{~s} 2)$

$\mathrm{da}=\operatorname{diag}(\mathrm{y} 1-\mathrm{pi} 1)$

$\mathrm{db}=\operatorname{diag}(\mathrm{y} 2-\mathrm{pi} 2)$ 


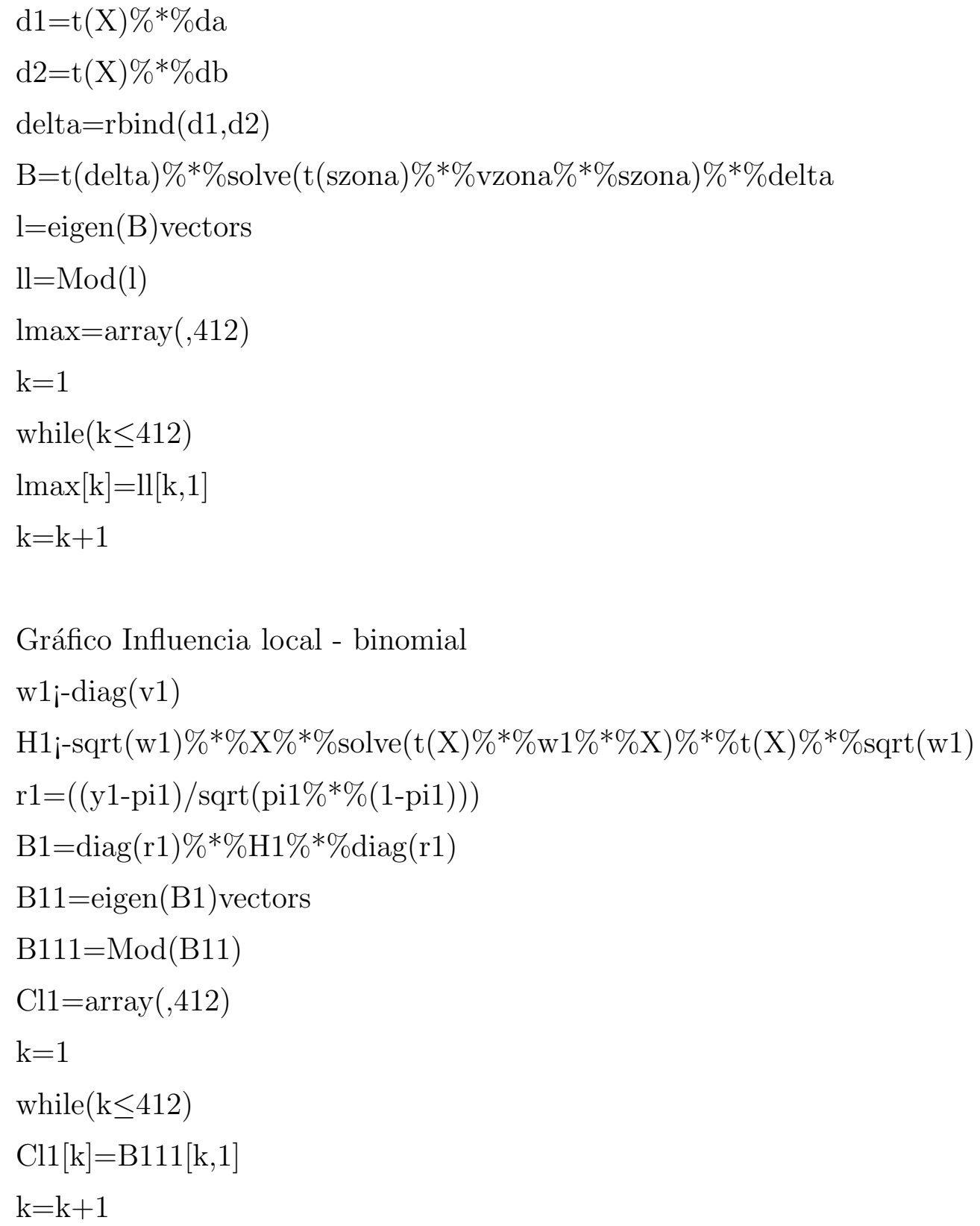


$\mathrm{k}=1$

while $(\mathrm{k} \leq 412) \quad \mathrm{C}[\mathrm{k}]=2^{*} \operatorname{Mod}(\mathrm{B}[\mathrm{k}, \mathrm{k}])$

$\mathrm{k}=\mathrm{k}+1$

Cbarra $=2^{*} \operatorname{mean}(\mathrm{C})$

Gráfico Influencia local total - binomial

$\mathrm{v} 1=\mathrm{pi}^{*}(1-\mathrm{pi} 1)$

$\mathrm{v} 2=$ pi $2 *(1-$ pi 2$)$

$\mathrm{v} 3=-\mathrm{pi} 1 * \mathrm{p} 12$

$\mathrm{w} 1 j-\operatorname{diag}(\mathrm{v} 1)$

$\mathrm{H} 1 \mathrm{j}$-sqrt(w1)\%*\%X\%*\%solve(t(X)\%*\%w1\%*\%X)\%*\%t(X)\%*\%sqrt(w1) h1j-diag(H1)

w2j-diag(v2)

$\mathrm{r} 1=((\mathrm{y} 1-\mathrm{pi} 1) / \operatorname{sqrt}(\mathrm{pi1} \% * \%(1-\mathrm{pi} 1)))$

Ci1=array $(, 412)$

$\mathrm{k}=1$

while $(\mathrm{k} \leq 412)$

$\mathrm{Ci} 1[\mathrm{k}]=2^{*}(\mathrm{r} 1[\mathrm{k}] \% * \% \mathrm{r} 1[\mathrm{k}] \% * \% \mathrm{~h} 1[\mathrm{k}])$

$\mathrm{k}=\mathrm{k}+1$

C1barra $=2 *$ mean $(\mathrm{C} 1)$

Gráfico Componente do desvio - trinomial

$\mathrm{v} 1=\mathrm{pi} 1 *(1-\mathrm{pi} 1)$

$\mathrm{v} 2=$ pi2 $*(1-$ pi2 $)$

$\mathrm{v} 3=-\mathrm{pi} 1 * \mathrm{p} 12$

$\mathrm{w} 1=\operatorname{diag}(\mathrm{v} 1)$

$\mathrm{H} 1=\operatorname{sqrt}(\mathrm{w} 1) \% * \% \mathrm{X} \% * \% \operatorname{solve}(\mathrm{t}(\mathrm{X}) \% * \% \mathrm{w} 1 \% * \% \mathrm{X}) \% * \% \mathrm{t}(\mathrm{X}) \% * \% \operatorname{sqrt}(\mathrm{w} 1)$

$\mathrm{h} 1=\operatorname{diag}(\mathrm{H} 1)$ 
$\mathrm{w} 2=\operatorname{diag}(\mathrm{v} 2)$

$\mathrm{H} 2=\operatorname{sqrt}(\mathrm{w} 2) \% * \% \mathrm{X} \% * \%$ solve $(\mathrm{t}(\mathrm{X}) \% * \% \mathrm{w} 2 \% * \% \mathrm{X}) \% * \% \mathrm{t}(\mathrm{X}) \% * \% \operatorname{sqrt}(\mathrm{w} 2)$

$\mathrm{h} 2=\operatorname{diag}(\mathrm{H} 2)$

$\operatorname{lp} 0=\operatorname{sqrt}\left(-2^{*} \log (\mathrm{pi} 0)\right)$

$\operatorname{lp} 1=\operatorname{sqrt}\left(-2^{*} \log (\right.$ pi1 $\left.)\right)$

$\operatorname{lp} 2=\operatorname{sqrt}\left(-2^{*} \log (\mathrm{pi} 2)\right)$

$\mathrm{d} 0=\operatorname{lp} 0 * \mathrm{y} 0$

$\mathrm{d} 1=\operatorname{lp} 1 * \mathrm{y} 1$

$\mathrm{d} 2=\operatorname{lp} 2 * \mathrm{y} 2$

$\mathrm{d}=\mathrm{d} 0+\mathrm{d} 1+\mathrm{d} 2$

hmedio $=\operatorname{sqrt}(1-(\mathrm{h} 1+\mathrm{h} 2) / 2)$

$\operatorname{td}=\operatorname{sort}(\mathrm{d} /$ hmedio $)$

Gráfico de Envelope - trinomial

O gráfico de foi gerado para os resíduos componente do desvio por simulação, após gerar 20 amostras aleatórias de $Y_{i j}, i=0,1,2$ e $j=1, \ldots, 412$, estimamos as estimativas de $\pi_{1}$ e $\pi_{2}$ para as 20 amostras no SAS, a seguir transportamos essas estimativas para o S-plus. A partir desse ponto é só proceder como se fosse gerar 20 gráficos componente do desvio, após gerar os 20 vetores poderemos gerar o envelope como segue.

$\operatorname{tdi}=\operatorname{cbind}(\operatorname{td} 1, \operatorname{td} 2, \ldots, \operatorname{td} 20)$

$\mathrm{TD}=\mathrm{t}(\mathrm{tdi})$

$\mathrm{v}=\operatorname{array}(, 412)$

$\mathrm{ve}=\operatorname{array}(, 412)$

vet $=\operatorname{array}(, 412)$

Med=array $(, 412)$

$\mathrm{k}=1$

$\mathrm{PQ}=\operatorname{array}(, 412)$ 
$\mathrm{TQ}=\operatorname{array}(, 412)$

while $(\mathrm{k} \leq 412)$

$l=1$

while $(1 \leq 20)$

$\mathrm{v}[\mathrm{l}] \mathrm{i}-\mathrm{TD}[\mathrm{l}, \mathrm{k}]$

$l=l+1$

$\mathrm{ve}=\operatorname{sort}(\mathrm{v})$

$\operatorname{Med}[\mathrm{k}]=\operatorname{median}(\mathrm{ve})$

$\mathrm{PQ}[\mathrm{k}]=\mathrm{ve}[2]$

$\mathrm{TQ}[\mathrm{k}]=\mathrm{ve}[19]$

$\mathrm{k}=\mathrm{k}+1$

faixa=range $(\mathrm{td}, \mathrm{PQ}, \mathrm{TQ})$ - td é o vetor de componente desvio original

$\operatorname{par}(\mathrm{pty}=" \mathrm{~s} ")$

qqnorm(td,xlab="Percentis da N(0,1)",ylab="Componente do Desvio",ylim=faixa)

$\operatorname{par}($ new $=2)$

qqnorm $(P Q$, axes $=F$, xlab=,ylab=,main $=$, type $=" 1$, ylim=faixa,lty $=1)$

$\operatorname{par}($ new $=2)$

qqnorm $(T Q$, axes $=F$, xlab=,ylab=,main=,type="l",ylim=faixa,lty=1)

$\operatorname{par}($ new $=2)$

qqnorm $($ Med,axes $=F$, xlab $=$, ylab $=$, main $=$, type $=" l ", y l i m=$ faixa,lty $=2)$ 


\section{Bibliografia}

Agresti A. (1990). Categorical Data Analysis. John Wiley, New York.

Atkinson, A. C. (1981). Two graphical display for outlying and influential observations in regression. Biometrika 68, 13-20.

Atkinson, A. C. (1985). Plots, Transformations and Regressions. Oxford Statistical Science Series, Oxford.

Beckman, R. J.; Nachtsheim, C. J. e Cook, R. D. (1987). Diagnostics for mixed-model analysis of variance. Technometrics $\mathbf{2 9}$, 413-426.

Belsley, D. A.; Kuh, E. e Welsch, R. E. (1980). Regression Diagnostics. John Wiley, New York.

Chatterjee, S. e Hadi, A. S. (1988). Sensitivity Analysis in Linear Regression. New York: Wiley.

Chatterjee, S. e Hadi, A. S. (1986). Influential observations, high leverage points, and outliers in linear regression. Statistical Science, 379- 416.

Collett, D. (1991). Modelling Binary Data. Chapman and Hall, London.

Cook, R. D. (1977). Detection of influential observations in linear regressions. Technometrics 19, 15-18. 
Cook, R. D. (1986). Assessment of local influence (with discussion). Journal of the Royal Statistical Society B 48, 133-169.

Cook, R. D.; Peña, D. e Weisberg, S. (1988). The likelihood displacement: A unifying principle for influence measures. Communications in Statistics, Theory and Methods 17, 623-640

Cook, R. D. e Weisberg, S. (1982). Residuals and Influence in Regression. Chapman and Hall, London.

Cordeiro, G. M. e Paula, G. A. (1992). Estimation, large-sample parametric tests and diagnostics for non-exponential family nonlinear models. Communications in Statistics, Simula 21, 149-172.

Cox, D. R. e Snell, E. J. (1968). A General definition of residuals (with discussion). Journal of the Royal Statistical Society B 30, 248-275.

Cox, D. R. e Snell, E. J. (1989). The Analysis of Binary Data, 2nd Edition. Chapman and Hall, London.

Davison, A. C. e Gigli, A. (1989). Deviance residuals and normal scores plots. Biometrika 76, 211-221.

de Souza, F. A. M. e Paula, G. A. (2002). Assessing local influence in mixed von Mises regression models. Australian and New Zealand Journal of Statistics 44, 345-356.

Davison, A.C. e Tsai, C-L. (1992). Regression model diagnostics. International Statistical Review 60, 337-353.

Escobar, L. A. e Meeker, W. Q. (1992). Assessing influence in regression analysis with censored data. Biometrics 48, 507-528. 
Fahrmeir, L. e Tutz, G. (1994). Multivariate Statistical Modelling based on Generalized Linear Models. Springer, New York.

Forthofer, R. N. e Lehnen, R. G.(1981). Public program analysis, A new categoric data approach. Lifetime Learning Publications 16, 21.

Fung, W. K. (1993). Unmasking outliers and leverage points: a confirmation. Journal of the American Statistical Association 88, 515-519.

Fung, W. K. e Kwan, C. W. (1997). A note on local influence based on normal curvature. Journal of the Royal Statistical Society B 59, 839-843.

Galea, M; Paula, G. A. e Bolfarine, H. (1997). Local influence in elliptical linear regression models. The Statistician 46, 71-79.

Galea, M; Paula, G. A. e Uribe-Opazo, M. (2003). On influence diagnostics in univariate elliptical linear regression models. Statistical Papers 44, 23-45.

Galea, M.; Riquelme, M. e Paula, G. A. (2000). Diagnostic methods in elliptical linear regression models. Brazilian Journal of Probability and Statistics 14, 167-184.

Gu, H. e Fungi W. K. (1998). Assessing local influence in canonical correlation analysis. Annals of the Institute of Statistical Mathematics 50, 755-772.

Kwan, C. W. e Fung, W. K. (1998). Assessing local influence for specific restricted likelihood: Applications to factor analysis. Psychometrika 63, 35-46.

Lesaffre, F. E. e Verbeke, G. (1998). Local influence in linear mixed models. Biometrics 54, 579-582.

Hoaglin, D. C. e Welsch, R. E. (1978). The hat matrix in regression and ANOVA. The American Statistician 32, 17-22. 
Hosmer, D. W. e Lemeshow, S. (1989). Applied Logistic Regression. John Wiley, New York.

Kim, M. G. (1995). Local influence in multivariate regression. Communications in Statistics, Theory and Methods 20, 1271-1278.

Lawrence, A. J. (1988). Regression transformation diagnostics using local influence. Journal of the American Statistical Association 84, 124-141.

Liu, S. Z. (2000). On local influence for elliptical linear models. Statistical Papers 41, 211-224.

McCullagh, P. (1987). Tensor Methods in Statistics. Chapman and Hall, London.

McCullagh, P. e Nelder, J. A. (1989). Generalized Linear Models, 2nd. Edition. Chapman and Hall, London.

Moolgavkar, S. H., Lustbader, E. D. e Venzon, D. J. (1984). A geometric approach to non-linear regression diagnostics with application to matched case-control studies. Annals of Statistics 12, 816-826.

O’Hara Hines, J.; Lawless, J. F. e Carter, E. M. (1992). Diagnostics for a cumulative multinomial genralized linear model with application to grouped toxicological mortality data. Journal of the American Statistical Association 87, 1059-1069.

Ortega, E. M. M.; Bolfarine, H. e Paula, G. A. (2003). Influence diagnostic in generalized log-gamma regression models. Computational Statistics and Data Analysis 42, 165186.

Pan, J. X.; Fang, K. T. e von Rosen, D. (1997). Local influence assessment in the growth curve model with unstructured covariance. Journal of Statistical Planning and Inference 62, 263-278. 
Paula, G. A. (1993). Assessing local influence in restricted regression moldels. Computational Statistics and Data Analysis 16, 63-79.

Paula, G. A. (1995). Influence and residuals in restricted generalized linear models. Journal of Statistical Computation and Simulation 51, 315-352.

Paula, G. A. (1996). Influence diagnostic in proper dispersion models. Australian Journal of Statistics 38, 307-316.

Paula, G. A. (1999). Leverage in inequality constrained regression models. The Statistician 48, 529-538.

Paula, G. A. (2003). Modelos de Regressão: Com Apoio Computacional. Versão Preliminar, 300 p., IME-USP.

Paula, G. A. e Peres, C. A. (1988). Diagnostics for GLMs with linear inequality parameter constraints. Communications in Statistics, Theory and Methods 17, 4205-4219.

Peña, D. e Yohai, V. (1999). A fast procedure for outlier diagnostics in large regression problems. Journal of the American Statistical Association 94, 434-445.

Pettitt, A. N. e Bin Daud, I. (1989). Case-weight measures of influence for proportional hazards regression. Applied Statistics 38, 51-67.

Pregibon, D. (1981). Logistic regression diagnostics. Annals of Statistics 9, 705-724.

Ross, W. H.(1987). The geometry of case deletion and the assessment of influence in nonlinear regression. Canadian Journal of Statistics 15, 91-103.

Silva, G. L. (1992). Modelos Logísticos para Dados Binários. Dissertação de Mestrado, IME-USP.

St. Laurent, R. T. e Cook, R. D. (1992). Leverage and superleverage in nonlinear regression. Journal of the American Statistical Association, 87, 985-990. 
Svetliza, C. F. e Paula, G. A. (2003). Diagnostics in nonlinar negative binomial models. Communications in Statistics, Theory and Methods 32, 1227-1250.

Tsai, C. H. e Wu, X. (1992). Assessing local influence in linear regression models with first-order autoregressive or heteroscedastic error structure. Statistics and Probability Letters 14, 247-252.

Wei, B.C., Hu, Y.Q. e Fung, W.K. (1998). Generalized leverage and its applications. Scandinavian Journal of Statistics 25, 25-37.

Williams, D. A. (1984). Residuals in generalized linear models. In: Proceedings of the 12th. International Biometrics Conference, Tokyo, pp. 59-68.

Williams, D. A. (1987). Generalized linear model diagnostic using the deviance and single case deletion. Applied Statistics 36, 181-191. 